\title{
Invariant measures for the 3D globally modified Navier-Stokes equations with unbounded variable delays
}

\author{
Jintao Wang ${ }^{a *}, \quad$ Caidi Zhao ${ }^{b \dagger}, \quad$ Tomás Caraballo ${ }^{c \ddagger}$ \\ ${ }^{a}$ Center for Mathematical Sciences, Huazhong University of Science and Technology, \\ Wuhan, Hubei Province, 430074, People's Republic of China \\ ${ }^{b}$ Department of Mathematics, Wenzhou University, \\ Wenzhou, Zhejiang Province, 325035, People's Republic of China \\ ${ }^{c}$ Departmento de Ecuaciones Diferenciales y Análisis Numérico, Facultad de Matemáticas, \\ Universidad de Sevilla, c/Tarfia s/n, 41012-Sevilla, Spain
}

March 30, 2020

\begin{abstract}
This article investigates the three-dimensional globally modified Navier-Stokes equations with unbounded variable delays. Firstly, we prove the global well-posedness of the solutions, and give the existence of the pullback attractor for the associated process. Then, we construct a family of invariant Borel probability measures, which is supported by the pullback attractor.
\end{abstract}

Keywords: Globally modified Navier-Stokes equations; Unbounded variable delays; Invariant measures; Pullback attractors.

MSC2010: 35B41, 34D35, 76F20

\section{Introduction}

In this article, we study the following three-dimensional (3D) system of globally modified NavierStokes (GMNS for short) equations with unbounded variable delays

$$
\begin{cases}\frac{\partial u}{\partial t}-\nu \Delta u+F_{N}\left(\|u\|_{V}\right)[(u \cdot \nabla) u]+\nabla p=f(x, t)+g(t, u(t-\rho(t))) & \text { in }(\tau,+\infty) \times \Omega \\ \nabla \cdot u=0 & \text { in }(\tau,+\infty) \times \Omega \\ u=0 & \text { on }(\tau,+\infty) \times \partial \Omega \\ u(\tau+s, x)=\phi(s, x) & \text { in }(-\infty, 0] \times \Omega,\end{cases}
$$

where the unknown functions $u=u(x, t)$ and $p=p(x, t)$ denote, respectively, the velocity field of the fluid and the pressure, the positive constant $\nu$ is the viscosity coefficient, $f(x, t)$ is the density of the

\footnotetext{
${ }^{*}$ Corresponding author E-mail: wangjt@ hust.edu.cn

†E-mail: zhaocaidi2013@163.com or zhaocaidi@wzu.edu.cn

${ }^{\ddagger}$ E-mail: caraball@us.es
} 
volume forces, $g$ is the external force affected by memory during the time range $(-\infty, \tau], \rho: \mathbb{R} \rightarrow \mathbb{R}^{+}$ is a delay function satisfying $\rho \in L_{\mathrm{loc}}^{\infty}(\mathbb{R})$ and $\phi$ is the initial datum on the interval $(-\infty, 0]$. Here $\|u\|_{V}=\|\nabla u\|_{\left(L^{2}(\Omega)\right)^{3}}$ and the function $F_{N}(\cdot):(0,+\infty) \mapsto(0,1]$ is defined as

$$
F_{N}(r):=\min \{1, N / r\}, \quad r \in(0,+\infty),
$$

where $N$ is a positive integer. In addition, $\Omega \subset \mathbb{R}^{3}$ is an open bounded set with smooth boundary $\partial \Omega$.

The GMNS equations with $g \equiv 0$ (that is without delays) was firstly formulated in [5]. It is one kind of global modifications of the Navier-Stokes (NS for short) equations with the modifying factor $F_{N}\left(\|u\|_{V}\right)$ depending on the $\left(L^{2}(\Omega)\right)^{3}$-norm of $\nabla u$. This modification brings some good properties to the solutions: global existence, uniqueness and regularity. The GMNS equations are useful in obtaining some new results for the 3D NS equations. For instance, it was used in [5] to establish the existence of bounded entire weak solutions for the 3D NS equations, and in [19], the authors applied it to show weak compactness and weak connectedness of the attainability set of the weak solutions of the 3D NS equations satisfying an energy inequality. At the same time, It was proved in [5, 29] that the solutions of the GMNS equations converge to those of the 3D NS equations in some proper sense. Therefore, the GMNS equations (1.1) with $g \equiv 0$ can be regarded as an approximation to the 3D NS equations.

The GMNS equations (1.1) with $g \equiv 0$ have been extensively studied. For example, one can see $[5,6,12,17,33]$ for the existence and uniqueness of the weak and strong solutions, $[8,18,24,37]$ for invariant measure and statistical solutions, [39] for the existence of trajectory attractor and trajectory statistical solutions. However, the real world is inundated with many situations in which the model is better described with some terms containing delays appearing in the equations. These delays may appear, for instance, when the current behavior is influenced by its previous states, or one wants to control the system by imposing an external force which depends upon both the present state and the history of the solutions. The delayed partial differential equations are usually used to describe these delayed phenomena.

Nowadays, delayed partial differential equations have drawn much attention and have been extensively studied. For example, the existence and asymptotic behaviors of solutions for the NS equations with finite delays were investigated in $[2-4,14,25]$; the existence of pullback attractors for the NS equations with finite or infinite delays was studied in [7, 16,21,28]. Some known references concerning the delayed 3D GMNS equations are summarized as:

$1^{\circ}$ Finite delays: the existence, uniqueness and global asymptotic exponentially stability of the stationary solution, as well as the convergence of solutions to those of the NS equations were established in $[29,30]$.

$2^{\circ}$ Infinite delays: the well-posedness and global exponential decay of the solutions to the stationary solution, as well as the existence of pullback attractor were investigated in [26, 27].

$3^{\circ}$ Bounded variable delays: the existence and uniqueness of solutions, as well as the existence of pullback attractors were proved in [9,31].

However, up to our knowledge, there is no reference concerning the 3D GMNS equations with unbounded variable delays.

The motivation of this article is to investigate the existence of invariant Borel probability measures for the 3D GMNS equations with unbounded variable delays. The invariant measures and statistical 
solutions have played a significant role and developed into a crucial tool in the research of turbulence (see Foias et al. [13]) in recent decades. This is mainly due to the fact that some time-average quantities essentially measure several important aspects of turbulent flows. The invariant measures and statistical properties of dissipative systems were studied by a number of researchers after that. For instance, Wang considered the upper semi-continuity of stationary statistical properties for dissipative systems in [36]. Łukaszewicz, Real and Robinson in [23] constructed the invariant measures for general continuous dynamical systems on metric spaces by using the generalized Banach limit. For a much wider class of dissipative semigroups, Chekroun and Glatt-Holtz [11] also applied the generalized Banach limit to constructing the invariant measures, but they generalized and simplified the proofs of [23] and [36].

Recently, a series of works developed some techniques to provide a construction of invariant measures for non-autonomous systems with minimal assumptions on the underlying dynamical process (see Foias et al. [13], Wang [36] and Łukaszewicz et al. [22-24]). Nowadays, these theories have been employed to establish invariant measures for some evolution equations, see e.g. [20,32,37,44] and the references therein. However, as far as we know, there is no reference investigating the invariant measures for delayed evolution equations.

The main result of the current article is the existence of invariant Borel probability measures for the 3D GMNS equations with unbounded variable delays. We will use the abstract theory for dissipative non-autonomous system in [24, Theorem 3.1]. To this end, we shall prove that the solution operators associated to the problem (1.1) generate a continuous process $\{U(t, \tau)\}_{t \geqslant \tau}$ in the phase space $\mathcal{C}_{\gamma}(H)$ (see notation in Section 2) and

(1) the process $\{U(t, \tau)\}_{t \geqslant \tau}$ is pullback strongly bounded in $\mathcal{C}_{\gamma}(H)$;

(2) the process $\{U(t, \tau)\}_{t \geqslant \tau}$ is pullback asymptotically compact in $\mathcal{C}_{\gamma}(H)$;

(3) for each given $t_{*} \in \mathbb{R}$ and $\phi_{*} \in \mathcal{C}_{\gamma}(H)$, the $\mathcal{C}_{\gamma}(H)$-valued function $\tau \mapsto U\left(t_{*}, \tau\right) \phi_{*}$ is continuous and bounded on $(-\infty, t]$ (this is called $\tau$-continuity of the process $\{U(t, \tau)\}_{t \geqslant \tau}$ ).

We first use a Galerkin approximation argument, and some a priori estimates, to prove the global well-posedness of the solutions to the problem (1.1). Also we do some estimates of the solutions to obtain the existence of strongly bounded pullback absorbing sets. Secondly we apply the Ascoli-Arzelà type theorem ( [1]) to establish the pullback asymptotic compactness of the process $\{U(t, \tau)\}_{t \geqslant \tau}$. This is prompted by the delay appearing in the equations. Since the problem discussed contains unbounded variable delays, it seems suitable for us to choose $C_{\gamma}(H)$ as the phase space. It has been shown that the Ascoli-Arzelà theorem is a powerful tool to investigate the compactness of subsets of $C_{\gamma}(H)$. Thirdly, for each given $t_{*} \in \mathbb{R}$ and $\phi_{*} \in C_{\gamma}(H)$, the continuity of the $C_{\gamma}(H)$-valued function $\tau \mapsto U\left(t_{*}, \tau\right) \phi_{*}$ on $(-\infty, t]$ is not a clear fact (see Lemma 4.4 and the auxiliary lemmas). There are some essential differences between the autonomous dynamical system and non-autonomous one, say the continuous dependence of the dynamical system on their parameters. Even if we have known that the $C_{\gamma}(H)$ valued function $t \mapsto U(t, \tau) \phi_{*}$ is continuous on $\mathbb{R}$ for every given $\tau$ and $\phi_{*} \in C_{\gamma}(H)$, the convergence $\left\|U\left(t_{*}, \tau\right) \phi_{*}-\phi_{*}\right\|_{\gamma} \rightarrow 0$ as $\tau \rightarrow t_{*}^{-}$may still depend on $\tau$ itself. In fact, when $\tau \rightarrow t_{*}^{-}, U\left(t_{*}, \tau\right) \phi_{*}$ also changes with different initial times $\tau$. This is caused naturally by the non-autonomous and delayed phenomena. We will take advantage of the structure of the GMNS equations to prove the $\tau$-continuity of the process $\{U(t, \tau)\}_{t \geqslant \tau}$.

This article is organized as follows. In the next section, we first introduce some notations and oper- 
ators, and then prove the global well-posedness of solutions to the problem (1.1). Section 3 is devoted to the proof of the existence of pullback attractors. In Section 4, we first prove the $\tau$-continuity of the process and then construct a family of invariant Borel probability measures which is supported by the pullback attractors. We end the article with conclusions and remarks in Section 5.

\section{Global well-posedness of solutions}

In this section, we first introduce some notations and operators. Then we prove the global wellposedness of the problem (1.1).

In this article, we use the following notations:

$\mathbb{L}^{2}(\Omega)=\left(L^{2}(\Omega)\right)^{3}$-the 3D Lebesgue space with norm $\|\cdot\|_{\mathbb{L}^{2}(\Omega)}=\|\cdot\|$;

$\mathbb{H}^{1}(\Omega)=$ the $3 \mathrm{D}$ Sobolev space $\left\{\phi \in \mathbb{L}^{2}(\Omega) \mid \nabla \phi \in \mathbb{L}^{2}(\Omega)\right\}$ with norm $\|\nabla \cdot\|_{\mathbb{L}^{2}(\Omega)}$;

$\mathbb{H}_{0}^{1}(\Omega)=$ closure of $\left(\mathcal{C}_{0}^{\infty}(\Omega)\right)^{3}$ in $\mathbb{H}^{1}(\Omega)$ with norm $\|\cdot\|_{\mathbb{H}^{1}(\Omega)}$;

$\mathcal{V}=\left\{\phi \in\left(\mathcal{C}_{0}^{\infty}(\Omega)\right)^{3} \mid \nabla \cdot \phi=0\right\}$

$H=$ closure of $\mathcal{V}$ in $\mathbb{L}^{2}(\Omega)$ with norm $\|\cdot\|$ and inner product $(\cdot, \cdot)$;

$V=$ closure of $\mathcal{V}$ in $\mathbb{H}^{1}(\Omega)$ with norm $\|\cdot\|_{V}=\|\nabla \cdot\|_{\mathbb{L}^{2}(\Omega)}$;

$V^{\prime}=$ dual space of $V$ with norm $\|\cdot\|_{V^{\prime}} ;\langle\cdot, \cdot\rangle$ - the dual pairing between $V$ and $V^{\prime}$;

In addition, we will use the notation $a \lesssim b$ to mean that $a \leqslant c b$ for a universal constant $c>0$ that only depends on the parameters coming from the problem and will not result in confusion.

For short, we will write the problem (1.1) in a functional form. To this end, we introduce some classical operators which are usually used to handle the 3D incompressible NS and GMNS equations. At the same time, we will select some known estimates and properties (see e.g. [35]) of these operators.

We first consider the operator $A: V \rightarrow V^{\prime}$ defined as

$$
\langle A u, v\rangle=(\nabla u, \nabla v), \quad \forall u, v \in V,
$$

where $D(A)=\mathbb{H}^{2}(\Omega) \cap V$ and $\mathbb{H}^{2}(\Omega)=\left\{\phi \in \mathbb{H}^{1}(\Omega) \mid \Delta \phi \in \mathbb{L}^{2}(\Omega)\right\}$. In fact, for each $u \in D(A)$, $A u=-P \Delta u$, and hence $A$ is the Stokes operator, where $P$ is the Leray-Helmholtz projection from $\mathbb{L}^{2}(\Omega)$ onto $H$.

Secondly, we define a trilinear form $b(\cdot, \cdot, \cdot)$ as follows,

$$
b(u, v, w)=\sum_{i, j=1}^{3} \int_{\Omega} u_{i} \frac{\partial v_{j}}{\partial x_{i}} w_{j} \mathrm{~d} x, \quad \forall u, v, w \in \mathbb{H}_{0}^{1}(\Omega) .
$$

Notice that $V$ is a closed subspace of $\mathbb{H}_{0}^{1}(\Omega)$. One can check that $b(u, v, w)$ is continuous on $V \times V \times V$, and $b(u, v, w)=-b(u, w, v), b(u, v, v)=0, \forall u, v, w \in V$. For each $u, v \in V$,

$$
\langle B(u, v), w\rangle=b(u, v, w), \quad \forall w \in V,
$$

defines a continuous function $B(u, v)$ on $V \times V$. At the same time, we have (cf. [35])

$$
\begin{array}{ll}
\|b(u, v, w)\| \lesssim\|u\|_{V}^{1 / 2}\|A u\|^{1 / 2}\|v\|_{V}\|w\|, & \forall u \in D(A), v \in V, w \in H \\
\|b(u, v, w)\| \lesssim\|u\|_{V}\|v\|_{V}\|w\|_{V}^{1 / 2}\|w\|^{1 / 2}, & \forall u, v, w \in V .
\end{array}
$$


We further set

$$
\begin{aligned}
b_{N}(u, v, w) & =F_{N}\left(\|v\|_{V}\right) b(u, v, w), \quad \forall u, v, w \in V, \\
\left\langle B_{N}(u, v), w\right\rangle & =b_{N}(u, v, w), \quad \forall u, v, w \in V .
\end{aligned}
$$

Then $b_{N}(u, v, w)$ is linear in $u$ and $w$, but not linear in $v$. However, we still have (see [33])

$$
\begin{aligned}
b_{N}(u, v, v) & =0, \quad \forall u, v \in V, \\
\left|b_{N}(u, v, w)\right| & \lesssim N\|u\|_{V}\|w\|_{V}, \quad \forall u, v, w \in V, \\
\left|b_{N}(u, v, w)\right| & \lesssim N\|A u\|\|w\|, \quad \forall u \in D(A), v \in V, w \in H .
\end{aligned}
$$

Furthermore, for every $u, v \in V$ and each $N>0$,

$$
\begin{aligned}
0 & \leqslant\|u\|_{V} F_{N}\left(\|u\|_{V}\right) \leqslant N \\
\left|F_{N}\left(\|u\|_{V}\right)-F_{N}\left(\|v\|_{V}\right)\right| & \leqslant \frac{1}{N} F_{N}\left(\|u\|_{V}\right) F\left(\|v\|_{V}\right)\|u-v\|_{V} .
\end{aligned}
$$

To formulate the problem (1.1) in a proper manner, we require that the functions $\rho(t), f(x, t)$ and $g(t, u(t-\rho(t)))$ in (1.1) satisfy some natural hypotheses.

(H1) Suppose that the function $\rho: \mathbb{R} \mapsto \mathbb{R}_{+}$belongs to $L_{\mathrm{loc}}^{\infty}(\mathbb{R})$ and $f(t, x) \in L_{\mathrm{loc}}^{2}\left(\mathbb{R} ; \mathbb{L}^{2}(\Omega)\right)$. Also assume that $g: \mathbb{R} \times H \rightarrow H$ satisfies

(gl) for every $u \in H$, the mapping $\mathbb{R} \ni t \mapsto g(t, u)$ is measurable;

(g2) $g(t, 0)=0$ for all $t \in \mathbb{R}$;

(g3) there exists a positive constant $\gamma$ and a nonnegative function $L_{g}: \mathbb{R} \rightarrow \mathbb{R}_{+}$such that

$$
L_{g}(\cdot) e^{\gamma \rho(\cdot)} \in L_{\mathrm{loc}}^{2}(\mathbb{R}) \quad \text { and } \quad\|g(t, u)-g(t, v)\| \leqslant L_{g}(t)\|u-v\|, \quad \forall t \in \mathbb{R}, \forall u, v \in H .
$$

Remark 2.1. In Remark 3.1 we will give a concrete example to show the existence of the functions $\rho(t)$, $f(t, x), g(t, u), L_{g}(t)$ and the constant $\gamma$ that satisfy our assumption (H1). In the sequel, the constant $\gamma$ is from $(g 3)$.

In order to deal with the unbounded variable delays, we consider the space

$$
C_{\gamma}(H):=\left\{\phi \in C((-\infty, 0] ; H): \sup _{s \leqslant 0} e^{\gamma s}\|\phi(s)\|<+\infty, \exists \lim _{s \rightarrow-\infty} e^{\gamma s} \phi(s) \in H\right\},
$$

with the norm $\|\phi\|_{\gamma}:=\sup _{s \leqslant 0} e^{\gamma s}\|\phi(s)\|$. Then $\left(C_{\gamma}(H),\|\cdot\|_{\gamma}\right)$ is a Banach space. In addition, for each $\tau \in \mathbb{R}$ we denote conventionally $u_{\tau}(s)=u(\tau+s)$ with $s \in(-\infty, 0]$.

We now specify the definition of weak solution to the problem (1.1).

Definition 2.1. Let $\tau \in \mathbb{R}$ and $\phi \in C_{\gamma}(H)$ be given. If for all $T>\tau$, a function $u \in C((-\infty, T], H) \cap$ $L^{2}(\tau, T ; V)$ with $u_{\tau}=\phi$ satisfies

$$
\frac{\mathrm{d} u(t)}{\mathrm{d} t}+\nu A u(t)+B_{N}(u(t), u(t))=f(t)+g(t, u(t-\rho(t)),
$$

in the sense of distribution $\mathcal{D}^{\prime}\left(\tau,+\infty ; V^{\prime}\right)$, then we call $u$ a weak solution of the problem (1.1) corresponding to the initial datum $\phi$. 
For the existence and uniqueness of solutions to the problem (1.1), we have the following result.

Theorem 2.1. Let the assumption (H1) hold true. Then for each $\tau \in \mathbb{R}$ and $\phi \in C_{\gamma}(H)$, there corresponds a unique weak solution $u(\cdot)=u(\cdot ; \tau, \phi)$ to the problem (1.1), and $u$ is in fact a strong solution in the sense that

$$
u \in C((\tau, T], V) \cap L^{2}(\tau+\varepsilon, T ; D(A)), \quad \forall \varepsilon>0, \quad \forall T>\tau+\varepsilon .
$$

Moreover, if $\phi(0) \in V$, then

$$
u \in C([\tau, T] ; V) \cap L^{2}(\tau, T ; D(A)), \quad \forall T>\tau .
$$

Proof. The proof is similar to that of [26, Theorem 2] and [27, Theorem 1] with the unbounded variable delays in place of the infinite delays. Following the proof of [26, Theorem 2] with some different estimates, we present the parts that will be used in the sequel of this article.

For the Galerkin approximation solution $u^{(m)}$, we have

$$
\begin{aligned}
\frac{\mathrm{d}}{\mathrm{d} t}\left\|u^{(m)}(t)\right\|^{2}+2 \nu\left\|u^{(m)}(t)\right\|_{V}^{2} & =2\left(f(t), u^{(m)}(t)\right)+2\left(g\left(t, u^{(m)}(t-\rho(t))\right), u^{(m)}(t)\right) \\
& \lesssim \nu\left\|u^{(m)}(t)\right\|_{V}^{2}+\|f(t)\|^{2}+2 L_{g}(t)\left\|u^{(m)}(t-\rho(t))\right\|\left\|u^{(m)}(t)\right\| \\
& \lesssim \nu\left\|u^{(m)}(t)\right\|_{V}^{2}+\|f(t)\|^{2}+2 L_{g}(t) e^{\gamma \rho(t)}\left\|u_{t}^{m}\right\|_{\gamma}^{2},
\end{aligned}
$$

using the facts that $\left\|u^{(m)}(t)\right\|=\left\|u_{t}^{(m)}(0)\right\| \leqslant\left\|u_{t}^{(m)}\right\|_{\gamma}$ and

$$
\left\|u^{(m)}(t-\rho(t))\right\|=e^{\gamma \rho(t)} e^{-\gamma \rho(t)}\left\|u^{(m)}(t-\rho(t))\right\| \leqslant e^{\gamma \rho(t)}\left\|u_{t}^{(m)}\right\|_{\gamma} .
$$

Hence, for all $t \geqslant \tau$ there holds

$$
\left\|u^{(m)}(t)\right\|^{2}+\nu \int_{\tau}^{t}\left\|u^{(m)}(s)\right\|_{V}^{2} \mathrm{~d} s \lesssim\left\|u^{(m)}(\tau)\right\|^{2}+\int_{\tau}^{t}\left(\|f(s)\|^{2}+2 L_{g}(s) e^{\gamma \rho(s)}\left\|u_{s}^{(m)}\right\|_{\gamma}^{2}\right) \mathrm{d} s .
$$

By (2.13) and the definition of the norm $\left\|u_{t}^{(m)}\right\|_{\gamma}^{2}$, we have

$$
\begin{aligned}
\left\|u_{t}^{(m)}\right\|_{\gamma}^{2} & \lesssim \max \left\{\sup _{\theta \leqslant \tau-t} e^{2 \gamma \theta}\|\phi(\theta+t-\tau)\|^{2}\right. \\
& \lesssim \max \left\{\sup _{\theta \in[\tau-t, 0]}\left(e^{2 \gamma \theta}\|u(\tau)\|^{2}+e^{2 \gamma \theta} \int_{\tau \leqslant 0}^{t+\theta}\left(\|f(s)\|^{2}+2 L_{g}(s) e^{\gamma \rho(s)}\left\|u_{s}^{(m)}\right\|_{\gamma}^{2}\right) \mathrm{d} s\right)\right\} \\
& \lesssim\|\phi\|_{\gamma}^{2}+\int_{\tau}^{t}\left(\|f(s)\|^{2}+2 L_{g}(s) e^{\gamma \rho(s)}\left\|u_{s}^{(m)}\right\|_{\gamma}^{2}\right) \mathrm{d} s .
\end{aligned}
$$

Applying Gronwall's lemma to (2.14) gives

$$
\left\|u_{t}^{(m)}\right\|_{\gamma}^{2} \lesssim\left(\|\phi\|_{\gamma}^{2}+\int_{\tau}^{t}\|f(s)\|^{2} \mathrm{~d} s\right) \exp \left(2 \int_{\tau}^{t} L_{g}(s) e^{\gamma \rho(s)} \mathrm{d} s\right)
$$


We then conclude from (2.13) and (2.15) that, with the assumption (H1) in hand,

$$
\begin{aligned}
& \left\|u_{t}^{(m)}\right\|_{\gamma}^{2}+\left\|u^{(m)}\right\|_{L^{2}(\tau, T ; V)}^{2} \lesssim 1, \quad \forall t \in[\tau, T], \forall\|\phi\|_{\gamma} \leqslant R, \forall m \geqslant 1, \\
& \int_{\tau}^{T}\left\|g\left(s, u^{(m)}(s-\rho(s))\right)\right\|^{2} \mathrm{~d} s \leqslant \int_{\tau}^{T}\left(L_{g}(s) e^{\gamma \rho(s)}\right)^{2}\left\|u_{s}^{(m)}\right\|_{\gamma}^{2} \mathrm{~d} s \lesssim\left\|L_{g} e^{\gamma \rho}\right\|_{L^{2}(\tau, T)}^{2} .
\end{aligned}
$$

Estimate (2.16) implies that

$$
g\left(\cdot, u^{(m)}(\cdot-\rho(\cdot))\right) \in L^{2}\left(\tau, T ; \mathbb{L}^{2}(\Omega)\right) .
$$

The rest tasks are to prove that the Galerkin approximate solutions $\left\{u^{(m)}\right\}$ possess a convergent subsequence and its limiting function is the solution satisfying Theorem 2.1. The procedures are similar to those of [26, Theorem 2] and we omit the details here.

The result below shows that the solutions to (1.1) depend continuously on the initial data.

Theorem 2.2. Let the assumption (H1) hold. Let $u=u(\cdot ; \tau, \phi)$ and $\tilde{u}=\tilde{u}(\cdot ; \tau, \tilde{\phi})$ be two solutions of the problem (1.1) with the initial data $\phi$ and $\tilde{\phi}$ at the initial time $\tau$, respectively. Then we have

$$
\begin{aligned}
& \max _{\theta \in[\tau, t]}\|u(\theta)-\tilde{u}(\theta)\|^{2} \\
\lesssim & \|\phi(0)-\tilde{\phi}(0)\|^{2} \exp \left(\int_{\tau}^{t} 2 L_{g}(s) e^{\gamma \rho(s)} \mathrm{d} s+(t-\tau)\right) \\
& +\|\phi-\tilde{\phi}\|_{\gamma}^{2} \int_{\tau}^{t} L_{g}(s) e^{\gamma \rho(s)} \exp \left(\int_{\tau}^{s} 2 L_{g}(\theta) e^{\gamma \rho(\theta)} \mathrm{d} \theta+(s-\tau)\right) \mathrm{d} s .
\end{aligned}
$$

The proof of Theorem 2.2 is analogous to that of [27, Proposition 1] and we omit the details.

\section{Existence of the pullback attractors}

From Theorems 2.1 and 2.2 we can conclude that the solution operators $U(t, \tau): C_{\gamma}(H) \rightarrow C_{\gamma}(H)$ defined by

$$
U(t, \tau) \phi(s)=u_{t}(s), \quad \tau \leqslant t, \quad s \in(-\infty, 0]
$$

generate a continuous process $\{U(t, \tau)\}_{t \geqslant \tau}$ on the phase space $C_{\gamma}(H)$, where $u=u(\cdot ; \tau, \phi)$ is the solution of the problem (1.1) corresponding to the initial function $\phi$ at the initial time $\tau$. In this section, we first prove that $\{U(t, \tau)\}_{t \geqslant \tau}$ possesses a bounded pullback absorbing set and is pullback asymptotically compact in $C_{\gamma}(H)$. Then we obtain the existence of the pullback attractor. For related definitions and results concerning the evolution processes and pullback attractors, we can refer to $[4,10,34]$ and the references therein.

In the sequel, we pick $\gamma$ and some parameter $\mu$ such that

$$
0<\mu<\nu \text { and }(\nu-\mu) \lambda_{1}<\gamma
$$

where $\lambda_{1}=\inf _{v \in V \backslash\{0\}} \frac{\|v\|_{V}^{2}}{\|v\|^{2}}>0$ is the first eigenvalue of the Stokes operator $A$. To obtain the existence of the bounded pullback absorbing set, we need another assumption for the functions $L_{g}(\cdot) e^{\gamma \rho(\cdot)}$ and $f$. 
(H2) Assume there is a constant $\mu$ satisfying (3.2) such that

$$
\begin{aligned}
& \underset{t \in \mathbb{R}}{\operatorname{ess} \sup } L_{g}(t) e^{\gamma \rho(t)}<(\nu-\mu) \lambda_{1} \leqslant \gamma, \\
& \int_{-\infty}^{0} e^{\sigma s}\|f(s)\|^{2} \mathrm{~d} s<+\infty
\end{aligned}
$$

hereinafter the constant $\sigma$ is given by

$$
\sigma:=2(\nu-\mu) \lambda_{1}-2 \underset{t \in \mathbb{R}}{\operatorname{ess} \sup } L_{g}(t) e^{\gamma \rho(t)} .
$$

Remark 3.1. We set

$$
\left\{\begin{array}{l}
\rho(t)=|t|, \quad t \in \mathbb{R} \\
g(t, u)=\exp \left(-e^{|t|}\right) u, \quad t \in \mathbb{R}, u \in H \\
L_{g}(t)=\exp \left(-e^{|t|}\right), \quad t \in \mathbb{R} \\
\gamma=e
\end{array}\right.
$$

then the conditions in (H1) are satisfied and

$$
\underset{t \in \mathbb{R}}{\operatorname{ess} \sup } L_{g}(t) e^{\gamma \rho(t)}=e^{\gamma \ln \gamma-\gamma}=e^{0}=1<e=\gamma .
$$

If we take $1<\nu<e$ and $\mu$ small enough, then (3.3) are satisfied. Thus, the functions and constant presented by (3.6) satisfy the conditions in (H1) and (H2). For the existence of the function $f(x, t)$ satisfying (H1) and (H2), we refer to [38, Example 3.1].

We denote by $\mathcal{P}\left(C_{\gamma}(H)\right)$ the family of all subsets of $C_{\gamma}(H)$ and consider the families of nonempty sets $\widehat{D}=\{D(t): t \in \mathbb{R}\} \subseteq \mathcal{P}\left(C_{\gamma}(H)\right)$. Let $\mathcal{D}_{\sigma}$ be the nonempty class of families $\widehat{D}=\{D(t): t \in$ $\mathbb{R}\} \subseteq \mathcal{P}\left(C_{\gamma}(H)\right)$ parameterized by time $t$ and satisfying

$$
\lim _{\tau \rightarrow-\infty}\left(e^{\sigma \tau} \sup _{v \in D(\tau)}\|v\|_{\gamma}^{2}\right)=0 .
$$

The class $\mathcal{D}_{\sigma}$ defined above is always called a tempered universe.

Lemma 3.1. Let the assumptions (H1) and (H2) hold. Then the process $\{U(t, \tau)\}_{t \geqslant \tau}$ possesses a bounded pullback absorbing set in $C_{\gamma}(H)$.

Proof. Pick some $\mu$ satisfying (3.2), and let $u=u(\cdot ; \tau, \phi)$ be the solution of the problem (1.1) corresponding to the initial function $\phi$ at the initial time $\tau$. Then we can get

$$
\frac{\mathrm{d}}{\mathrm{d} t}\|u(t)\|^{2}+2(\nu-\mu) \lambda_{1}\|u(t)\|^{2}+\mu\|u(t)\|_{V}^{2} \lesssim\|f(t)\|^{2}+2 L_{g}(t) e^{\gamma \rho(t)}\left\|u_{t}\right\|_{\gamma}^{2}, \quad t>\tau .
$$

Hence for all $t \geqslant \tau$,

$$
\begin{aligned}
& \|u(t)\|^{2}+\mu \int_{\tau}^{t} e^{-2(\nu-\mu) \lambda_{1}(t-s)}\|u(s)\|_{V}^{2} \mathrm{~d} s \\
\lesssim & e^{-2(\nu-\mu) \lambda_{1}(t-\tau)}\|u(\tau)\|^{2}+\int_{\tau}^{t} e^{-2(\nu-\mu) \lambda_{1}(t-s)}\left(\|f(s)\|^{2}+2 L_{g}(s) e^{\gamma \rho(s)}\left\|u_{s}\right\|_{\gamma}^{2}\right) \mathrm{d} s .
\end{aligned}
$$


Inequality (3.8) implies that

$$
\begin{aligned}
\left\|u_{t}\right\|_{\gamma}^{2} \lesssim \max \left\{\sup _{\theta \in(-\infty, \tau-t]} e^{2 \gamma \theta}\|\phi(\theta+t-\tau)\|^{2}, \sup _{\theta \in[\tau-t, 0]}\left(e^{2 \gamma \theta-2(\nu-\mu) \lambda_{1}(t+\tau-\theta)}\|u(\tau)\|^{2}\right.\right. \\
\left.\left.+e^{2 \gamma \theta} \int_{\tau}^{t+\theta} e^{-2(\nu-\mu) \lambda_{1}(t+\theta-s)}\left(\mid f(s)\left\|^{2}+2 L_{g}(s) e^{\gamma \rho(s)}\right\| u_{s} \|_{\gamma}^{2}\right) \mathrm{d} s\right)\right\} .
\end{aligned}
$$

By the choice of $\mu$ in (3.2), we have

$$
\begin{aligned}
& \sup _{\theta \in(-\infty, \tau-t]} e^{2 \gamma \theta}\|\phi(\theta+t-\tau)\| \leqslant e^{-(\nu-\mu) \lambda_{1}(t-\tau)}\|\phi\|_{\gamma}, \\
& \sup _{\theta \in[\tau-t, 0]} e^{2 \gamma \theta-2(\nu-\mu) \lambda_{1}(t+\tau-\theta)}\|u(\tau)\|^{2} \leqslant e^{-2(\nu-\mu) \lambda_{1}(t-\tau)}\|u(\tau)\|^{2}, \\
& \sup _{\theta \in[\tau-t, 0]} e^{2 \gamma \theta} \int_{\tau}^{t+\theta} e^{-2(\nu-\mu) \lambda_{1}(t+\theta-s)}\left(\|f(s)\|^{2}+2 L_{g}(s) e^{\gamma \rho(s)}\left\|u_{s}\right\|_{\gamma}^{2}\right) \mathrm{d} s \\
& \leqslant \int_{\tau}^{t} e^{-2(\nu-\mu) \lambda_{1}(t-s)}\left(\|f(s)\|^{2}+2 L_{g}(s) e^{\gamma \rho(s)}\left\|u_{s}\right\|_{\gamma}^{2}\right) \mathrm{d} s .
\end{aligned}
$$

It then follows from (3.9)-(3.12) that

$$
\left\|u_{t}\right\|_{\gamma}^{2} \lesssim e^{-2(\nu-\mu) \lambda_{1}(t-\tau)}\|\phi\|_{\gamma}^{2}+\int_{\tau}^{t} e^{-2(\nu-\mu) \lambda_{1}(t-s)}\left(\|f(s)\|^{2}+2 L_{g}(s) e^{\gamma \rho(s)}\left\|u_{s}\right\|_{\gamma}^{2}\right) \mathrm{d} s .
$$

Applying Gronwall's inequality to (3.13) yields

$$
\begin{aligned}
\left\|u_{t}\right\|_{\gamma}^{2} \lesssim & \|\phi\|_{\gamma}^{2} \exp \left\{-2(\nu-\mu) \lambda_{1}(t-\tau)+2 \int_{\tau}^{t} L_{g}(s) e^{\gamma \rho(s)} \mathrm{d} s\right\} \\
& +\int_{\tau}^{t}\|f(s)\|^{2} \exp \left\{-2(\nu-\mu) \lambda_{1}(t-s)+2 \int_{s}^{t} L_{g}(\sigma) \mathrm{e}^{\gamma \rho(\sigma)} \mathrm{d} \sigma\right\} \mathrm{d} s \\
= & \|\phi\|_{\gamma}^{2} e^{-\sigma(t-\tau)}+\int_{\tau}^{t}\|f(s)\|^{2} e^{-\sigma(s-\tau)} \mathrm{d} s
\end{aligned}
$$

Now we define the time-dependent family $\hat{\mathcal{B}}=\{\mathcal{B}(t): t \in \mathbb{R}\} \in \mathcal{P}\left(C_{\gamma}(H)\right)$ as

$$
\mathcal{B}(t)=\left\{v \in C_{\gamma}(H):\|v\|_{\gamma}^{2} \lesssim 1+\int_{-\infty}^{t} e^{-\sigma(t-s)}\|f(s)\|^{2} \mathrm{~d} s\right\}
$$

Then $\hat{\mathcal{B}}$ is the desired pullback $\mathcal{D}_{\sigma}$-absorbing set for $\{U(t, \tau)\}_{t \geqslant \tau}$ in $C_{\gamma}(H)$.

To establish the pullback $\mathcal{D}_{\sigma}$-asymptotic compactness for $\{U(t, \tau)\}_{t \geqslant \tau}$ in $C_{\gamma}(H)$, we will employ the generalized Ascoli-Arzelà type theorem ( [1]) to prove the following auxiliary lemma.

Lemma 3.2. Let $\left\{\tau_{n}\right\}_{n \geqslant 1}$ be a sequence such that $\tau_{n} \rightarrow-\infty$ as $n \rightarrow+\infty$ and $\tau_{n} \leqslant t_{0}$ for some fixed $t_{0} \in \mathbb{R}$, and $\left\{\phi_{n}\right\}_{n \geqslant 1}$ a sequence of functions with $\phi_{n} \in \mathcal{B}\left(\tau_{n}\right)$ for each positive integer $n$. Denote by $u^{(n)}=u^{(n)}\left(\cdot ; \tau_{n}, \phi_{n}\right)$ the solutions of the problem (1.1) corresponding to the initial data $\phi_{n}$ at the initial time $\tau_{n}$. Then there exist a subsequence (still denoted the same) $\left\{u_{t_{0}}^{(n)}\right\}_{n \geqslant 1}$ and a function $\psi \in C_{\gamma}(H)$ such that $u_{t_{0}}^{(n)} \rightarrow \psi$ in $C([-\bar{T}, 0] ; H)$ for each $\bar{T}>0$. 
Proof. Let $t_{0},\left\{\tau_{n}\right\}_{n \geqslant 1},\left\{\phi_{n}\right\}_{n \geqslant 1}$ and $u^{(n)}=u^{(n)}\left(\cdot ; \tau_{n}, \phi_{n}\right)$ be given as in this lemma. Consider two arbitrary positive numbers $\bar{T}, T$ with $T>\bar{T}$. Let $n_{0}=n_{0}\left(t_{0}, T\right)$ be the positive integer such that $\tau_{n}<t_{0}-T$ for all $n \geqslant n_{0}$. From (3.3) and (3.14)-(3.15) we deduce that for $t \in\left[t_{0}-T, t_{0}\right]$ and $n \geqslant n_{0}$,

$$
\begin{aligned}
\left\|u^{n}(t)\right\|^{2} & \lesssim R\left(t_{0}, T\right):=1+e^{-\sigma\left(t_{0}-T\right)} \int_{-\infty}^{t_{0}} e^{\sigma s}\|f(s)\|^{2} \mathrm{~d} s<+\infty \\
\left\|u_{t}^{n}\right\|_{\gamma}^{2} \lesssim R\left(t_{0}, T\right) . &
\end{aligned}
$$

We next divide two steps to establish that the conditions of the Ascoli-Arzelà type theorem are satisfied.

Step one. We first prove that

$$
\left\{u^{(n)}(t)\right\}_{n \geqslant n_{0}} \text { is precompact in } H \text { for each } t \in\left[t_{0}-\bar{T}, t_{0}\right] .
$$

By Theorem 2.1, we conclude that $u^{(n)}(t) \in D(A)$ for all $t \in\left[t_{0}-T, t_{0}\right]$, whence,

$$
\begin{aligned}
& \frac{1}{2} \frac{\mathrm{d}}{\mathrm{d} t}\left\|u^{(n)}(t)\right\|_{V}^{2}+\nu\left\|A u^{(n)}(t)\right\|^{2}+b_{N}\left(u^{(n)}(t), u^{(n)}(t), A u^{(n)}(t)\right) \\
= & \left(f(t), A u^{(n)}(t)\right)+\left(g\left(t, u^{(n)}(t-\rho(t))\right), A u^{(n)}(t)\right), \quad t \in\left[t_{0}-T, t_{0}\right] .
\end{aligned}
$$

Using Young's inequality, (2.1) and (2.6), we have

$$
\begin{aligned}
& \left(f(t), A u^{(n)}(t)\right) \lesssim \frac{\nu}{8}\left\|A u^{(n)}(t)\right\|^{2}+\|f(t)\|^{2}, \\
& \left(g\left(t, u^{(n)}(t-\rho(t))\right), A u^{(n)}(t)\right) \lesssim \frac{\nu}{8}\left\|A u^{(n)}(t)\right\|^{2}+L_{g}^{2}(t) e^{2 \gamma \rho(t)}\left\|u_{t}^{(n)}\right\|_{\gamma}^{2}, \\
& \left|b_{N}\left(u^{(n)}(t), u^{(n)}(t), A u^{(n)}(t)\right)\right| \lesssim\left\|u^{(n)}(t)\right\|_{V}^{1 / 2}\left\|A u^{(n)}(t)\right\|^{3 / 2} \lesssim \frac{\nu}{4}\left\|A u^{(n)}(t)\right\|^{2}+\left\|u^{(n)}(t)\right\|_{V}^{2} .
\end{aligned}
$$

Combining (3.19)-(3.22) gives

$$
\frac{\mathrm{d}}{\mathrm{d} t}\left\|u^{(n)}\right\|_{V}^{2}+\nu\left\|A u^{(n)}(t)\right\|^{2} \lesssim\|f(t)\|^{2}+2 L_{g}^{2}(t) e^{2 \gamma \rho(t)}\left\|u_{t}^{(n)}\right\|_{\gamma}^{2}+\left\|u^{(n)}(t)\right\|_{V}^{2} .
$$

Integrating (3.23) over $[s, t]$ with $t_{0}-T \leqslant s \leqslant t \leqslant t_{0}$ yields

$$
\left\|u^{(n)}(t)\right\|_{V}^{2} \lesssim\left\|u^{(n)}(s)\right\|_{V}^{2}+\int_{t_{0}-T}^{t_{0}}\left[\left(\|f(\theta)\|^{2}+2 L_{g}^{2}(\theta) e^{2 \gamma \rho(\theta)}\left\|u_{\theta}^{(n)}\right\|_{\gamma}^{2}\right)+\left\|u^{(n)}(\theta)\right\|_{V}^{2}\right] \mathrm{d} \theta .
$$

Now integrating (3.24) with respect to $s$ over $\left[t_{0}-T, t\right]$, we obtain for $t \in\left[t_{0}-\bar{T}, t_{0}\right]$ that

$$
\begin{aligned}
& (T-\bar{T})\left\|u^{(n)}(t)\right\|_{V}^{2} \leqslant\left(t+T-t_{0}\right)\left\|u^{(n)}(t)\right\|_{V}^{2} \\
\lesssim & T \int_{t_{0}-T}^{t_{0}}\left\|u^{(n)}(s)\right\|_{V}^{2} \mathrm{~d} s+T \int_{t_{0}-T}^{t_{0}}\left(\|f(s)\|^{2}+L_{g}^{2}(s) e^{2 \gamma \rho(s)}\left\|u_{s}^{(n)}\right\|_{\gamma}^{2}\right) \mathrm{d} s .
\end{aligned}
$$

In addition, from (3.8) and (3.14), we can get

$$
\begin{aligned}
\int_{\tau}^{t}\left\|u^{(n)}(s)\right\|_{V}^{2} \mathrm{~d} s \lesssim & \left(1+\int_{\tau}^{t} L_{g}(s) e^{\gamma \rho(s)} e^{2 \int_{\tau}^{s} L_{g}(\sigma) e^{\gamma \rho(\sigma)} \mathrm{d} \sigma} \mathrm{d} s\right)\|\phi\|_{\gamma}^{2} \\
& +\int_{\tau}^{t} e^{-2(\nu-\mu) \lambda_{1}(\tau-s)}\|f(s)\|^{2} \mathrm{~d} s \\
& +\int_{\tau}^{t} L_{g}(s) e^{\gamma \rho(s)} \int_{\tau}^{s} e^{-2(\nu-\mu) \lambda_{1}(\tau-\sigma)+2 \int_{\sigma}^{s} L_{g}(\theta) e^{\gamma \rho(\theta)} \mathrm{d} \theta}\|f(\sigma)\|^{2} \mathrm{~d} \sigma \mathrm{d} s .
\end{aligned}
$$


Note that we have $u^{(n)}\left(t ; \tau^{(n)}, \phi_{(n)}\right)=U\left(t, t_{0}-T\right) u\left(t_{0}-T ; \tau_{n}, \phi_{(n)}\right)$. Thus from (3.8)-(3.9), (3.13) and (3.15), we know that

$$
\text { the sequence }\left\{u^{(n)}\right\}_{n \geqslant n_{0}} \text { is bounded in } L^{\infty}\left(t_{0}-\bar{T}, t_{0} ; V\right) \text {. }
$$

Therefore, it follows from the compact embedding $V \hookrightarrow H$ and the continuity of $u^{(n)}$ (see (2.10)) that the set

$$
\left\{u^{n}(t): t \in\left[t_{0}-\bar{T}, t_{0}\right], n \geqslant n_{0}\right\} \text { is precompact in } H,
$$

from which (3.18) immediately follows.

Step two. We establish the equicontinuity of $\left\{u^{(n)}\right\}_{n \geqslant n_{0}}$ on $\left[t_{0}-\bar{T}, t_{0}\right]$ by contradiction.

Suppose that this equicontinuity does not hold true, and then there exists a positive constant $\varepsilon_{0}$ and two sequences $\left\{t_{n}^{(1)}\right\}$ and $\left\{t_{n}^{(2)}\right\}$, satisfying $t_{0}-\bar{T} \leqslant t_{n}^{(1)} \leqslant t_{n}^{(2)} \leqslant t_{0}$ and $\left|t_{n}^{(2)}-t_{n}^{(1)}\right| \leqslant \frac{1}{n}$, such that

$$
\left\|u^{(n)}\left(t_{n}^{(1)}\right)-u^{(n)}\left(t_{n}^{(2)}\right)\right\| \geqslant \varepsilon_{0} .
$$

By (3.28), we can assume that $t_{n}^{(1)} \rightarrow t^{*}, u^{(n)}\left(t^{*}\right) \rightarrow z^{*}$ and $u^{(n)}\left(t_{n}^{(i)}\right) \rightarrow z^{(i)}(i=1,2)$ in $H$ as $n \rightarrow+\infty$. Evidently, $t_{n}^{(2)} \rightarrow t^{*}$ as $n \rightarrow+\infty$ and $t^{*} \in\left[t_{0}-\bar{T}, t_{0}\right]$. By (3.23), we know that

$$
\left\|z^{(1)}-z^{(2)}\right\| \geqslant \varepsilon_{0} .
$$

Set $y^{(n)}(t)=u^{(n)}(t)-u^{(n)}\left(t^{*}\right)$ for $n \geqslant n_{0}$. Then we obtain

$$
\begin{aligned}
& \frac{1}{2} \frac{\mathrm{d}}{\mathrm{d} t}\left\|y^{(n)}(t)\right\|^{2}+\nu\left\|y^{(n)}(t)\right\|_{V}^{2}+\nu \int_{\Omega} \nabla u^{(n)}\left(t^{*}\right) \cdot \nabla y^{(n)}(t) \mathrm{d} x+b_{N}\left(u^{(n)}(t), u^{(n)}(t), y^{(n)}(t)\right) \\
= & \left(f(t), y^{(n)}(t)\right)+\left(g\left(t, u^{(n)}(t-\rho(t))\right), y^{(n)}(t)\right), \quad t \in\left[t_{0}-\bar{T}, t_{0}\right] .
\end{aligned}
$$

Applying Schwartz's inequality and (g3), we have

$$
\begin{aligned}
\left|\int_{\Omega} \nabla u^{(n)}\left(t^{*}\right) \cdot \nabla y^{(n)}(t) \mathrm{d} x\right| & \leqslant \frac{1}{2}\left\|u^{(n)}\left(t^{*}\right)\right\|_{V}^{2}+\frac{1}{2}\left\|y^{(n)}(t)\right\|_{V}^{2}, \\
2\left|\left(f(t), y^{(n)}(t)\right)\right| & \leqslant\|f(t)\|^{2}+\left\|y^{(n)}(t)\right\|^{2}, \\
2\left|\left(g\left(t, u^{(n)}(t-\rho(t))\right), y^{(n)}(t)\right)\right| & \leqslant L_{g}^{2}(t) e^{2 \gamma \rho(t)}\left\|u_{t}^{(n)}\right\|_{\gamma}^{2}+\left\|y^{(n)}(t)\right\|^{2} .
\end{aligned}
$$

At the same time, by (2.3)-(2.6) and (2.7), we see that

$$
\begin{aligned}
\left.\mid b_{N}\left(u^{(n)}(t), u^{(n)}(t)\right), y^{(n)}(t)\right) \mid & =\left|b_{N}\left(y^{(n)}(t), u^{(n)}(t), y^{(n)}(t)\right)-b_{N}\left(u^{(n)}\left(t^{*}\right), u^{(n)}(t), u^{(n)}\left(t^{*}\right)\right)\right| \\
& \lesssim\left\|y^{(n)}(t)\right\|_{V}^{3 / 2}\left\|y^{(n)}(t)\right\|^{1 / 2}+\left\|u^{(n)}\left(t^{*}\right)\right\|_{V}^{2} \\
& \lesssim \frac{\nu}{2}\left\|y^{(n)}(t)\right\|_{V}^{2}+\left\|y^{(n)}(t)\right\|^{2}+\left\|u^{(n)}\left(t^{*}\right)\right\|_{V}^{2}
\end{aligned}
$$

Taking (3.31)-(3.35) into account yields

$$
\frac{\mathrm{d}}{\mathrm{d} t}\left\|y^{(n)}(t)\right\|^{2} \lesssim\left\|y^{(n)}(t)\right\|^{2}+\|f(t)\|^{2}+L_{g}^{2}(t) e^{2 \gamma \rho(t)}\left\|u_{t}^{(n)}\right\|_{\gamma}^{2}+\left\|u^{(n)}\left(t^{*}\right)\right\|_{V}^{2} .
$$


Integrating (3.36) from $t^{*}$ to $t_{n}^{(i)}$ and using (3.16)- (3.17) gives

$$
\begin{aligned}
\left\|y^{(n)}\left(t_{n}^{i}\right)\right\|^{2} \leqslant & {\left[R\left(t_{0}, T\right)+c_{1}\right]\left|t_{n}^{i}-t^{*}\right|+\left|\int_{t^{*}}^{t_{n}^{i}}\|f(s)\|^{2} \mathrm{~d} s\right| } \\
& +R\left(t_{0}, T\right)\left|\int_{t^{*}}^{t_{n}^{i}} L_{g}^{2}(s) e^{2 \gamma \rho(s)} \mathrm{d} s\right|
\end{aligned}
$$

where (see (2.10) and (3.27))

$$
c_{1}:=\sup _{t \in\left[t_{0}-\bar{T}, t_{0}\right]}\left\{\left\|u_{n}(t)\right\|_{V}: n \geqslant n_{0}\right\}
$$

Letting $n \rightarrow+\infty$ in (3.37) and using the assumptions $f \in L_{\text {loc }}^{2}\left(\mathbb{R} ; \mathbb{L}^{2}(\Omega)\right)$ and (g3), we conclude that

$$
\left\|z^{i}-z^{*}\right\|^{2}=\lim _{n \rightarrow+\infty}\left\|u^{(n)}\left(t_{n}^{i}\right)-u^{(n)}\left(t^{*}\right)\right\|^{2}=0, \quad \text { for } i=1,2,
$$

which contradicts (3.30).

At this stage, we can apply the Ascoli-Arzelà type theorem [1] to claim that for each $\bar{T}>0$, $\left\{u^{(n)}\right\}_{n \in \mathbb{N}_{+}}$is precompact in $C\left(\left[t_{0}-\bar{T}, t_{0}\right] ; H\right)$. Thus there exists a function $\psi \in C([-\bar{T}, 0] ; H)$ and a subsequence of $u_{t_{0}}^{(n)}$ such that $\left.u_{t_{0}}^{(n)}\right|_{[-\bar{T}, 0]} \rightarrow \psi$ in $C([-\bar{T}, 0] ; H)$. Repeating this procedure for $n \bar{T}$ with $n=2,3, \cdots$, and using the diagonal procedure, we can obtain a function $\psi \in C((-\infty, 0] ; H)$ such that (extract a subsequence if necessary) $\left.u_{t_{0}}^{(n)}\right|_{[-\bar{T}, 0]} \rightarrow \psi$ in $C([-\bar{T}, 0] ; H)$ for each positive number $\bar{T}$. The proof of Lemma 3.2 is complete.

Lemma 3.3. Let the assumptions (H1) and (H2) hold. Then the process $\{U(t, \tau)\}_{t \geqslant \tau}$ is pullback $\mathcal{D}_{\sigma^{-}}$ asymptotically compact in $C_{\gamma}(H)$.

Proof. We continue to use the notations of the previous lemma. To establish the pullback $\mathcal{D}_{\sigma}$-asymptotic compactness, we shall prove that $u_{t_{0}}^{n} \rightarrow \psi$ in $C_{\gamma}(H)$, for which, it is sufficient to show that, for every $\varepsilon>0$, there exists some positive integer $n_{\varepsilon}$ such that

$$
\sup _{s \in(-\infty, 0]} e^{2 \gamma s}\left\|u_{t_{0}}^{(n)}(s)-\psi(s)\right\|^{2}<\varepsilon, \quad \text { for all } n \geqslant n_{\varepsilon} .
$$

Indeed, by (3.16)-(3.17) and the convergence proved in the previous lemma, we have that

$$
\|\psi(s)\|^{2} \lesssim 1+e^{-\sigma\left(t_{0}-T\right)} \int_{-\infty}^{t_{0}} e^{\sigma s}\|f(s)\|^{2} \mathrm{~d} s=R\left(t_{0}, T\right), \quad \text { for all } s \in[-T, 0] \text { and } T>0 .
$$

The rest proof is similar to that of [27, Proposition 3] and we omit the details here.

At this stage we combine Lemmas 3.1, 3.3 and the general result of [15, Theorem 3.11] to obtain the main result of this section, namely,

Theorem 3.1. Let the assumptions $(\mathbf{H 1})$ and $(\mathbf{H 2})$ hold. Then the process $\{U(t, \tau)\}_{t \geqslant \tau}$ possesses a pullback $\mathcal{D}_{\sigma}$-attractor (denoted by) $\hat{\mathcal{A}}_{\mathcal{D}_{\sigma}}^{H}=\left\{\mathcal{A}_{\mathcal{D}_{\sigma}}^{H}(t): t \in \mathbb{R}\right\}$ in $C_{\gamma}(H)$. 


\section{Existence of the invariant Borel probability measures in $C_{\gamma}(H)$}

In this section, we first aim to prove that for each given $t \in \mathbb{R}$ and $\phi \in C_{\gamma}(H)$, the $C_{\gamma}(H)$-valued function $\tau \mapsto U(t, \tau) \phi$ is bounded and continuous on $(-\infty, t]$. Then we combine Theorem 3.1 and the abstract result of [24, Theorem 3.1] to obtain the existence of invariant Borel probability measures for $\{U(t, \tau)\}_{t \geqslant \tau}$ in $C_{\gamma}(H)$.

Lemma 4.1. Let the assumptions (H1) and (H2) hold. Then for every $\phi \in C_{\gamma}(H)$ and every $t \in \mathbb{R}$ the $C_{\gamma}(H)$-valued function $\tau \mapsto U(t, \tau) \phi$ is bounded on $(-\infty, t]$.

Proof. Let $\phi \in C_{\gamma}(H)$ and $t \in \mathbb{R}$ be given. Note (3.4) is equivalent to (see [27, Remark 5])

$$
\int_{-\infty}^{t} e^{\sigma s}\|f(s)\|^{2} \mathrm{~d} s<+\infty \quad \text { for all } t \in \mathbb{R} .
$$

Then (3.14) and (4.1) imply that for all $\tau \in(-\infty, t]$,

$$
\|U(t, \tau) \phi\|_{\gamma}^{2} \lesssim e^{-\sigma(t-\tau)}\|\phi\|_{\gamma}^{2}+\int_{\tau}^{t} e^{-\sigma(t-s)}\|f(s)\|^{2} \mathrm{~d} s \leqslant\|\phi\|_{\gamma}^{2}+e^{-\sigma t} \int_{-\infty}^{t} e^{\sigma s}\|f(s)\|^{2} \mathrm{~d} s
$$

the right-hand side of which is bounded by a positive constant independent of $\tau \in(-\infty, t]$.

In the sequel, we set a positive number $M(t, \phi)$ such that

$$
M^{2}(t, \phi):=\|\phi\|_{\gamma}^{2}+e^{-\sigma t} \int_{-\infty}^{t} e^{\sigma s}\|f(s)\|^{2} \mathrm{~d} s .
$$

Then $M^{2}(t, \phi)$ is a continuous function with respect to $t \in \mathbb{R}$.

To establish the continuity of the $C_{\gamma}(H)$-valued function $\tau \mapsto U(t, \tau) \phi$ on $(-\infty, t]$, we next prove two auxiliary lemmas.

Lemma 4.2. Let $\phi \in C_{\gamma}(H)$ be given. Then for each $\varepsilon>0$, there exists some $\delta_{1}=\delta_{1}(\varepsilon, \phi)>0$ such that for all $s_{1}, s_{2} \in(-\infty, 0]$ with $\left|s_{1}-s_{2}\right|<\delta_{1}$ there holds

$$
e^{\gamma s_{2}}\left\|\phi\left(s_{1}\right)-\phi\left(s_{2}\right)\right\|<\varepsilon
$$

Proof. By the definition of $C_{\gamma}(H)$, we set $\phi_{\infty}:=\lim _{s \rightarrow-\infty} e^{\gamma s} \phi(s) \in H$. Then there exists some $s_{0}<0$ such that

$$
\left\|e^{\gamma s} \phi(s)-\phi_{\infty}\right\|<\frac{\varepsilon}{4}, \forall s \leqslant s_{0}
$$

and thus

$$
\left\|e^{\gamma s_{1}} \phi\left(s_{1}\right)-e^{\gamma s_{2}} \phi\left(s_{2}\right)\right\| \leqslant\left\|e^{\gamma s_{1}} \phi\left(s_{1}\right)-\phi_{\infty}\right\|+\left\|e^{\gamma s_{2}} \phi\left(s_{2}\right)-\phi_{\infty}\right\|<\frac{\varepsilon}{2}, \forall s_{1}, s_{2} \leqslant s_{0} .
$$

On the other hand, the $H$-valued function $s \mapsto e^{\gamma s} \phi(s)$ is uniformly continuous on the interval $\left[s_{0}-1,0\right]$, whence there is some $\delta_{1}^{\prime} \in(0,1)$ such that

$$
\left\|e^{\gamma s_{1}} \phi\left(s_{1}\right)-e^{\gamma s_{2}} \phi\left(s_{2}\right)\right\|<\frac{\varepsilon}{2} \quad \text { for } s_{1}, s_{2} \in\left[s_{0}-1,0\right] \text { with }\left|s_{1}-s_{2}\right|<\delta_{1}^{\prime} .
$$


Letting $\delta_{1}=\min \left\{\delta_{1}^{\prime}, \frac{1}{\gamma} \ln \left(1+\frac{\varepsilon}{2\|\phi\|_{\gamma}}\right)\right\}$, we have by (4.3) and (4.4) that for all $s_{1}, s_{2} \in(-\infty, 0]$ with $\left|s_{1}-s_{2}\right|<\delta_{1}$ there holds

$$
\begin{aligned}
& e^{\gamma s_{2}}\left\|\phi\left(s_{1}\right)-\phi\left(s_{2}\right)\right\| \leqslant\left\|e^{\gamma s_{1}} \phi\left(s_{1}\right)-e^{\gamma s_{2}} \phi\left(s_{2}\right)\right\|+\left|e^{\gamma s_{1}}-e^{\gamma s_{2}}\right|\left\|\phi\left(s_{1}\right)\right\| \\
\leqslant & \frac{\varepsilon}{2}+\left|e^{\gamma\left(s_{2}-s_{1}\right)}-1\right|\|\phi\|_{\gamma}<\varepsilon .
\end{aligned}
$$

The proof is complete.

Lemma 4.3. Let the assumptions (H1) and (H2) hold, and $\tau_{*} \in \mathbb{R}$ and $\phi \in C_{\gamma}(H)$ be given. Then for each $\varepsilon>0$, there exists some $\delta_{2}=\delta_{2}\left(\varepsilon, \tau_{*}, \phi\right)>0$ such that

$$
\|u(s ; \tau, \phi)-\phi(0)\|<\varepsilon, \quad \text { for all } \tau \in\left(\tau_{*}-\delta_{2}, \tau_{*}\right) \text { and } s \in\left[\tau, \tau_{*}\right],
$$

where $u(s ; \tau, \phi)$ is the solution of the problem (1.1) with the initial datum $\phi$ at the initial time $\tau$.

Proof. Let $\tau_{*} \in \mathbb{R}$ and $\phi \in C_{\gamma}(H)$ be given. Firstly, we prove that

$$
\int_{\tau}^{\tau_{*}}\left\|\frac{\mathrm{d} u(\theta ; \tau, \phi)}{\mathrm{d} \theta}\right\|_{V^{\prime}}^{2} \mathrm{~d} \theta \leqslant c_{2}
$$

where

$$
c_{2}:=\|\phi\|_{\gamma}^{2}+\int_{\tau_{*}-1}^{\tau_{*}}\|f(\theta)\|^{2} \mathrm{~d} \theta+\sup _{\left[\tau_{*}-1, \tau_{*}\right]} M^{2}(\theta, \phi),
$$

is a bounded quantity depending on $\tau_{*}, g, f, \cdots$, etc., but being independent of $\tau \in\left[\tau_{*}-1, \tau_{*}\right]$. Indeed, from (2.9) we can see that

$$
\begin{gathered}
\left\|\frac{\mathrm{d} u(\theta ; \tau, \phi)}{\mathrm{d} \theta}\right\|_{V^{\prime}}^{2} \lesssim \\
+\|A u(\theta ; \tau, \phi)\|_{V^{\prime}}^{2}+\left\|B_{N}(u(\sigma ; \tau, \phi), u(\sigma ; \tau, \phi))\right\|_{V^{\prime}}^{2} \\
+\|f(\theta)\|^{2}+\|g(\theta, u(\theta-\rho(\theta)))\|^{2} .
\end{gathered}
$$

By (2.5), (g2), (g3) and some simple observations, we have

$$
\left\{\begin{array}{l}
\|A u(\theta ; \tau, \phi)\|_{V^{\prime}}^{2} \lesssim\|u(\theta ; \tau, \phi)\|_{V}^{2}, \\
\left\|B_{N}(u(\sigma ; \tau, \phi), u(\sigma ; \tau, \phi))\right\|_{V^{\prime}}^{2} \lesssim\|u(\theta ; \tau, \phi)\|_{V}^{2} \\
\|g(\theta, u(\theta-\rho(\theta)))\|^{2} \lesssim \sup _{\left[\tau_{*}-1, \tau_{*}\right]} M^{2}(\theta, \phi) .
\end{array}\right.
$$

Inserting (4.7) and (4.8) into (4.6), then using (3.3), (3.5) and (3.26) gives

$$
\begin{aligned}
\int_{\tau}^{\tau_{*}}\left\|\frac{\mathrm{d} u(\theta ; \tau, \phi)}{\mathrm{d} \theta}\right\|_{V^{\prime}}^{2} \mathrm{~d} \theta & \left.\lesssim \int_{\tau}^{\tau_{*}} \| u(\sigma ; \tau, \phi)\right)\left\|_{V}^{2} \mathrm{~d} \theta+\int_{\tau}^{\tau_{*}}\right\| f(\theta) \|_{V}^{2} \mathrm{~d} \theta+\left(\tau_{*}-\tau\right) \sup _{\left[\tau_{*}-1, \tau_{*}\right]} M^{2}(\theta, \phi) \\
& \lesssim\|\phi\|_{\gamma}^{2}+\int_{\tau_{*}-1}^{\tau_{*}}\|f(\theta)\|^{2} \mathrm{~d} \theta+\sup _{\left[\tau_{*}-1, \tau_{*}\right]} M^{2}(\theta, \phi), \forall \tau \in\left[\tau_{*}-1, \tau_{*}\right]
\end{aligned}
$$

that is (4.6) is proved. 
Secondly, we observe that for $\tau_{*}-1 \leqslant \tau<s \leqslant \tau_{*}$ there holds

$$
\begin{aligned}
& \|u(s ; \tau, \phi)-\phi(0)\|^{2} \\
= & \|u(s ; \tau, \phi)\|^{2}-\|\phi(0)\|^{2}-2(u(s ; \tau, \phi)-\phi(0), \phi(0)) \\
= & \int_{\tau}^{s} \frac{\mathrm{d}\|u(\theta ; \tau, \phi)\|^{2}}{\mathrm{~d} \theta} \mathrm{d} \theta-2(u(s ; \tau, \phi)-\phi(0), \phi(0)) .
\end{aligned}
$$

By (3.3) and (3.13), we obtain

$$
\begin{aligned}
\left|\int_{\tau}^{s} \frac{\mathrm{d}\|u(\theta ; \tau, \phi)\|^{2}}{\mathrm{~d} \theta} \mathrm{d} \theta\right| & \lesssim \int_{\tau}^{s}\|f(\theta)\|^{2} \mathrm{~d} \theta+\int_{\tau}^{s} L_{g}(\theta) e^{\gamma \rho(\theta)}\left\|u_{\theta}\right\|_{\gamma}^{2} \mathrm{~d} \theta \\
& \lesssim \int_{\tau}^{\tau_{*}}\|f(\theta)\|^{2} \mathrm{~d} \theta+\left(\tau_{*}-\tau\right) \sup _{\theta \in\left[\tau_{*}-1, \tau_{*}\right]} M^{2}(\theta, \phi) .
\end{aligned}
$$

Notice that $f \in L_{\mathrm{loc}}^{2}\left(\mathbb{R}, \mathbb{L}^{2}(\Omega)\right)$, and $\sup _{\theta \in\left[\tau_{*}-1, \tau_{*}\right]} M^{2}(\theta, \phi)$ is a constant independent of $\tau$. Therefore, from (4.11) we see that there exists some $\delta_{2}^{\prime}=\delta_{2}^{\prime}\left(\varepsilon, \tau_{*}, \phi\right) \in(0,1)$ such that

$$
\left|\int_{\tau}^{s} \frac{\mathrm{d}\|u(\theta ; \tau, \phi)\|^{2}}{\mathrm{~d} \theta} \mathrm{d} \theta\right|<\frac{\varepsilon^{2}}{2}, \quad \tau_{*}-\delta_{2}^{\prime}<\tau<s \leqslant \tau_{*} .
$$

At the same time, since $\phi(0) \in H$ and $V$ is dense in $H$, there exists some $\psi \in V$ such that

$$
\|\psi-\phi(0)\|<\varepsilon^{2} / 16 \sup _{\theta \in\left[\tau_{*}-1, \tau_{*}\right]} M^{2}(\theta, \phi) .
$$

Using (4.6),

$$
\begin{aligned}
|(u(s ; \tau, \phi)-\phi(0), \phi(0))| & \leqslant|(u(s ; \tau, \phi)-\phi(0), \phi(0)-\psi)|+|(u(s ; \tau, \phi)-\phi(0), \psi)| \\
& \leqslant 2 \sup _{\theta \in\left[\tau_{*}-1, \tau_{*}\right]} M(\tau, \phi)\|\phi(0)-\psi\|+|\langle u(s ; \tau, \phi)-\phi(0), \psi\rangle| \\
& <\frac{\varepsilon^{2}}{8}+\left|\left\langle\int_{\tau}^{s} \frac{\mathrm{d} u(\theta ; \tau, \phi)}{\mathrm{d} \theta} \mathrm{d} \theta, \psi\right\rangle\right| \\
& \leqslant \frac{\varepsilon^{2}}{8}+\|\psi\|_{V} \sqrt{\tau_{*}-\tau}\left(\int_{\tau}^{\tau_{*}}\left\|\frac{\mathrm{d} u(\theta ; \tau, \phi)}{\mathrm{d} \theta}\right\|_{V^{\prime}}^{2} \mathrm{~d} \theta\right)^{\frac{1}{2}} \\
& \leqslant \frac{\varepsilon^{2}}{8}+\sqrt{c_{2}}\|\psi\|_{V} \sqrt{\tau_{*}-\tau}
\end{aligned}
$$

which implies that there exists some $\delta_{2}^{\prime \prime} \in(0,1)$, independent of $\tau$, such that

$$
|(u(s ; \tau, \phi)-\phi(0), \phi(0))| \leqslant \frac{\varepsilon^{2}}{4}, \quad \tau_{*}-\delta_{2}^{\prime \prime}<\tau<s \leqslant \tau_{*} .
$$

Picking $\delta_{2}=\min \left\{\delta_{2}^{\prime}, \delta_{2}^{\prime \prime}\right\}$, the desired result follows from (4.10), (4.12) and (4.14). The proof of Lemma 4.3 is complete.

Lemma 4.4. Let the assumptions (H1) and (H2) hold. Then for every $\phi \in C_{\gamma}(H)$ and every $t \in \mathbb{R}$ the $C_{\gamma}(H)$-valued function $\tau \mapsto U(t, \tau) \phi$ is continuous on $(-\infty, t]$. 
Proof. Let $\phi \in C_{\gamma}(H)$ and $t \in \mathbb{R}$ be given. We shall prove that for each given $\tau_{*} \in(-\infty, t]$ the $C_{\gamma}(H)$-valued function $\tau \mapsto U(t, \tau) \phi$ is both left and right continuous at $\tau=\tau_{*}$. We next prove the left continuity at $\tau=\tau_{*}$.

Firstly, on one hand, for every $\varepsilon>0$ we deduce from Lemma 4.2 that there exists some $\delta_{3}^{\prime}=$ $\delta_{3}^{\prime}(\varepsilon, \phi)>0$ such that, for all $s_{1}, s_{2} \in(-\infty, 0]$ with $\left|s_{1}-s_{2}\right|<\delta_{1}$, there holds

$$
e^{\gamma s_{2}}\left\|\phi\left(s_{1}\right)-\phi\left(s_{2}\right)\right\|<\varepsilon / 2 \text {. }
$$

On the other hand, by Lemma 4.3 , there is a $\delta_{3}^{\prime \prime}=\delta_{3}^{\prime \prime}\left(\varepsilon, \tau_{*}, \phi\right)>0$ such that whenever $\tau \in\left(\tau_{*}-\delta_{3}^{\prime \prime}, \tau_{*}\right)$ there holds

$$
\|u(s ; \tau, \phi)-\phi(0)\|<\varepsilon / 2, \quad \text { for all } s \in\left[\tau, \tau_{*}\right] .
$$

Thus, for the positive $\varepsilon$ above, there exists some $\delta_{3}=\delta_{3}\left(\varepsilon, \tau_{*}, \phi\right)=\min \left\{\delta_{3}^{\prime}, \delta_{3}^{\prime \prime}\right\}>0$ such that

$$
\begin{aligned}
\left\|u_{*}(\theta)-u(\theta)\right\| & =\|\phi(\theta-\tau)-u(\theta)\| \\
& \leqslant\|\phi(\theta-\tau)-\phi(0)\|+\|\phi(0)-u(\theta ; \tau, \phi)\|<\varepsilon, \quad \tau_{*}-\delta_{3}<\tau<\tau_{*}, \quad \theta \in\left[\tau, \tau_{*}\right],
\end{aligned}
$$

which implies that

$$
\max _{\theta \in\left[\tau, \tau_{*}\right]}\left\|u_{*}\left(\theta ; \tau_{*}, \phi\right)-u(\theta ; \tau, \phi)\right\|<\varepsilon, \quad \tau_{*}-\delta_{3}<\tau<\tau_{*}, \quad \theta \in\left[\tau, \tau_{*}\right]
$$

where $u_{*}\left(\theta ; \tau_{*}, \phi\right)$ is the solution corresponding to the initial datum $\phi$ at the initial time $\tau_{*}$.

Secondly, by (2.18) and (3.3) we have

$$
\begin{aligned}
\max _{\theta \in\left[\tau_{*}, t\right]} & \left\|u_{*}\left(\theta ; \tau_{*}, \phi\right)-u(\theta ; \tau, \phi)\right\| \\
\lesssim & \left\|\phi(0)-u\left(\tau_{*} ; \tau, \phi\right)\right\|^{2} \exp \left(\int_{\tau_{*}}^{t} L_{g}(s) e^{\gamma \rho(s)} \mathrm{d} s+\left(t-\tau_{*}\right)\right) \\
& \quad+\left\|\phi-u_{\tau_{*}}\right\|_{\gamma}^{2} \int_{\tau_{*}}^{t} L_{g}(s) e^{\gamma \rho(s)} \exp \left(\int_{\tau_{*}}^{s} L_{g}(\theta) e^{\gamma \rho(\theta)} \mathrm{d} \theta+\left(s-\tau_{*}\right)\right) \mathrm{d} s . \\
& \lesssim\left\|\phi(0)-u\left(\tau_{*} ; \tau, \phi\right)\right\|^{2}+\left\|\phi-u_{\tau_{*}}\right\|_{\gamma}^{2},
\end{aligned}
$$

using the representation $u(\theta ; \tau, \phi)=u\left(\theta ; \tau_{*}, u\left(\tau_{*} ; \tau, \phi\right)\right)$ and the fact that $t$ and $\tau_{*}$ are fixed. Now by Lemma 4.3, we derive that for above $\varepsilon>0$ there exists $\delta_{4}^{\prime}=\delta_{4}^{\prime}\left(\varepsilon, t, \tau_{*}, \phi\right)>0$ such that

$$
\left\|\phi(0)-u\left(\tau_{*} ; \tau, \phi\right)\right\|<\varepsilon / 2, \text { for } \tau_{*}-\delta_{4}^{\prime}<\tau<\tau_{*} .
$$

At the same time, by (4.15) and (4.17), there exists $\delta_{4}^{\prime \prime}=\delta_{4}^{\prime \prime}\left(\varepsilon, \tau_{*}, \phi\right)>0$ such that when $\tau_{*}-\delta_{4}^{\prime \prime}<\tau<$ $\tau_{*}$ then

$$
\begin{aligned}
\left\|\phi-u_{\tau_{*}}\right\|_{\gamma} & \leqslant \max \left\{\sup _{s \leqslant \tau_{*}-\tau} e^{\gamma s}\left\|\phi(s)-u_{\tau_{*}}(s)\right\|, \sup _{s \in\left[\tau-\tau_{*}, 0\right]} e^{\gamma s}\left\|\phi(s)-u_{\tau_{*}}(s)\right\|\right\} \\
& \leqslant \max \left\{\sup _{s \leqslant \tau-\tau_{*}} e^{\gamma s}\left\|\phi(s)-\phi\left(s+\tau_{*}-\tau\right)\right\|, \sup _{s \in\left[\tau, \tau_{*}\right]}\left\|u_{*}(s)-u(s)\right\|\right\} \\
& \leqslant \max \left\{\sup _{r \leqslant 0} e^{\gamma\left(r+\tau-\tau_{*}\right)}\left\|\phi\left(r+\tau-\tau_{*}\right)-\phi(r)\right\|, \varepsilon\right\}<\varepsilon / 2 .
\end{aligned}
$$


Picking $\delta_{4}=\delta_{4}\left(\varepsilon, t, \tau_{*}, \phi\right)=\min \left\{\delta_{4}^{\prime}, \delta_{4}^{\prime \prime}\right\}$, then (4.18)-(4.20) imply

$$
\max _{\theta \in\left[\tau_{*}, t\right]}\left\|u_{*}\left(\theta ; \tau_{*}, \phi\right)-u(\theta ; \tau, \phi)\right\|<\varepsilon, \quad \tau_{*}-\delta_{4}<\tau<\tau_{*}<\varepsilon .
$$

We now choose $\delta=\delta\left(\varepsilon, t, \tau_{*}, \phi\right)=\min \left\{\delta_{3}, \delta_{4}\right\}$, and then deduce from (4.15), (4.17) and (4.21) that if $\tau_{*}-\delta<\tau<\tau_{*}$ then

$$
\begin{aligned}
& \left\|U(t, \tau) \phi-U\left(t, \tau_{*}\right) \phi\right\|_{\gamma}=\sup _{\theta \leqslant 0} e^{\gamma \theta}\left\|u(t+\theta)-u_{*}(t+\theta)\right\|_{\gamma} \\
\leqslant & \max \left\{\sup _{\theta \leqslant \tau-t} e^{\gamma \theta}\left\|\phi\left(\theta+t-\tau_{*}\right)-\phi(\theta+t-\tau)\right\|, \sup _{\theta \in[\tau-t, 0]} e^{\gamma \theta}\left\|u(t+\theta)-u_{*}(t+\theta)\right\|\right\} \\
\leqslant & \max \left\{\sup _{r \leqslant 0} e^{\gamma\left(r+\tau_{*}-t\right)}\left\|\phi(r)-\phi\left(r+\tau_{*}-\tau\right)\right\|, \sup _{\theta \in[\tau, t]}\left\|u(\theta)-u_{*}(\theta)\right\|\right\}<\varepsilon .
\end{aligned}
$$

The left continuity of the $C_{\gamma}(H)$-valued function $\tau \mapsto U(t, \tau) \phi$ at $\tau=\tau_{*}$ is proved. Since the proof of the right continuity of $U(t, \tau) \phi$ at $\tau=\tau_{*}$ is similar to that of the left continuity, we omit the details and end the proof of Lemma 4.4.

To construct the invariant measures for the process $\{U(t, \tau)\}_{t \geqslant \tau}$, we next recall the definition of generalized Banach limits.

Definition 4.1. ( $[13,24])$ A generalized Banach limit is any linear functional, denoted by $\mathrm{LIM}_{t \rightarrow+\infty}$, defined on the space of all bounded real-valued functions on $[0,+\infty)$ and satisfying

(1) $\operatorname{LIM}_{t \rightarrow+\infty} \zeta(t) \geqslant 0$ for nonnegative functions $\zeta(\cdot)$ on $[0,+\infty)$;

(2) $\operatorname{LIM}_{t \rightarrow+\infty} \zeta(t)=\lim _{t \rightarrow+\infty} \zeta(t)$ if the usual limit $\lim _{t \rightarrow+\infty} \zeta(t)$ exists.

Remark 4.1. Notice that we consider the "pullback" asymptotic behavior and we require generalized limits as $\tau \rightarrow-\infty$. For a given real-valued function $\zeta$ defined on $(-\infty, 0]$ and a given Banach limit $\operatorname{LIM}_{t \rightarrow+\infty}$, we define $\operatorname{LIM}_{t \rightarrow-\infty} \zeta(t)=\operatorname{LIM}_{t \rightarrow+\infty} \zeta(-t)$.

The main result of this article reads as follows.

Theorem 4.1. Let the assumptions (H1) and (H2) hold. Let $\{U(t, \tau)\}_{t \geqslant \tau}$ be the process associated to the problem (1.1) and $\widehat{\mathcal{A}}_{\mathcal{D}_{\sigma}}=\left\{\mathcal{A}_{\mathcal{D}_{\sigma}}(t): t \in \mathbb{R}\right\}$ the pullback $\mathcal{D}_{\sigma}$-attractor obtained in Theorem 3.1. Then for a given generalized Banach limit $\operatorname{LIM}_{t \rightarrow+\infty}$ and a continuous map $\xi_{\tau}: \mathbb{R} \longmapsto C_{\gamma}(H)$ with $\xi_{\tau}(\cdot) \in \mathcal{D}_{\sigma}$, there exists a unique family of Borel probability measures $\left\{m_{t}\right\}_{t \in \mathbb{R}}$ in $C_{\gamma}(H)$ such that the support of the measure $m_{t}$ is contained in $\mathcal{A}_{\mathcal{D}_{\sigma}}(t)$ and

$$
\begin{aligned}
\operatorname{LIM}_{\tau \rightarrow-\infty} \frac{1}{t-\tau} \int_{\tau}^{t} \Upsilon\left(U(t, s) \xi_{s}\right) \mathrm{d} s & =\int_{\mathcal{A}_{\mathcal{D}_{\sigma}}(t)} \Upsilon(u) \mathrm{d} m_{t}(u) \\
& =\int_{C_{\gamma}(H)} \Upsilon(u) \mathrm{d} m_{t}(u) \\
& =\operatorname{LIM}_{\tau \rightarrow-\infty} \frac{1}{t-\tau} \int_{\tau}^{t} \int_{C_{\gamma}(H)} \Upsilon(U(t, s) u) \mathrm{d} m_{s}(u) \mathrm{d} s
\end{aligned}
$$


for every real-valued continuous functional $\Upsilon$ on $C_{\gamma}(H)$. Moreover, $m_{t}$ is invariant in the sense that

$$
\int_{\mathcal{A}_{\mathcal{D}_{\sigma}}(t)} \Upsilon(u) \mathrm{d} m_{t}(u)=\int_{\mathcal{A}_{\mathcal{D}_{\sigma}}(\tau)} \Upsilon(U(t, \tau) u) \mathrm{d} m_{\tau}(u), \quad t \geqslant \tau .
$$

Proof. From Theorems 2.1 and 2.2, the solution operators of the problem (1.1) generate a continuous process $\{U(t, \tau)\}_{t \geqslant \tau}$ on the space $C_{\gamma}(H)$. Theorem 3.1 shows that $\{U(t, \tau)\}_{t \geqslant \tau}$ possesses a pullback attractor in $C_{\gamma}(H)$. Lemmas 4.1 and 4.4 indicate that for each given $t \in \mathbb{R}$ and $\phi \in C_{\gamma}(H)$ the $C_{\gamma}(H)$ valued function $\tau \mapsto U(t, \tau) \phi$ is bounded and continuous on $(-\infty, t]$. Using these facts and the abstract result of [24, Theorem 3.1], we obtain the results of Theorem 4.1.

\section{Conclusions and remarks}

In this article, we first prove the global well-posedness of the 3D GMNS equations with unbounded variable delays. Then we establish that its solution operators generate a continuous process $\{U(t, \tau)\}_{t \geqslant \tau}$ on the space $C_{\gamma}(H)$. Furthermore, the process $\{U(t, \tau)\}_{t \geqslant \tau}$ possesses a pullback attractor in $C_{\gamma}(H)$. Finally, we prove the existence of invariant Borel probability measures which are supported by the pullback attractor. There are two points that we would like to point out.

Firstly, we apply the generalized Ascoli-Arzelà theorem to prove the "weak version" of the pullback asymptotic compactness of the process $\{U(t, \tau)\}_{t \geqslant \tau}$ (see Lemma 3.2). This is prompted by the delay phenomenon. Since the problem discussed contains unbounded variable delays, it seems suitable for us to choose $C_{\gamma}(H)$ as the phase space. The Ascoli-Arzelà theorem has been proved to be a powerful tool to investigate the compactness of the sets such as the subset of $C_{\gamma}(H)$.

Secondly, for each given $t \in \mathbb{R}$ and $\phi \in C_{\gamma}(H)$, the continuity of the $C_{\gamma}(H)$-valued function $\tau \mapsto$ $U(t, \tau) \phi$ on $(-\infty, t]$ is not a clear fact (see Lemma 4.4 and the auxiliary lemmas). There are some essential differences between the autonomous dynamical system and non-autonomous one, say the continuous dependence of the dynamical system on their parameters. Consider a continuous process $\{S(t, \tau)\}_{t \geqslant \tau}$ on some Banach space $X$. Even if we have known that the $X$-valued function $t \mapsto S(t, \tau) x_{0}$ is continuous on $\mathbb{R}$ for every given $\tau$ and $x_{0} \in X$, the convergence $\left\|S\left(t_{*}, \tau\right) x_{0}-x_{0}\right\|_{X} \rightarrow 0$ as $\tau \rightarrow t_{*}^{-}$may still depend on $\tau$. In fact, when $\tau \rightarrow t_{*}^{-}, S\left(t_{*}, \tau\right) x_{0}$ changes simultaneously with different initial times $\tau$. This is caused naturally by the non-autonomous phenomenon.

In the end of this article, we want to propose a problem, saying, how to formulate reasonably and construct the statistical solutions for the evolution system containing delays. As for the 3D GMNS equations discussed here, we have constructed the invariant measures on the phase space $C_{\gamma}(H)$. However, we seem unable to construct its statistical solutions via the invariant measures and pullback attractors, as done in [40-43], because the invariant measures are contained in the phase space $C_{\gamma}(H)$ while the equation (2.9) is interpreted in the $\mathcal{D}\left(\tau,+\infty ; V^{\prime}\right)$ sense.

\section{Acknowledgement}

This work was supported by NSF of China with No.11801190, 11971356 and by NSF of Zhejiang Province with No.LY17A010011, and also supported by FEDER and the Spanish Ministerio de Ciencia, Innovación y Universidades project PGC2018-096540-B-I00. 


\section{References}

[1] J. P. Aubin, A. Cellina, Differential Inclusions, Springer-Verlag, New York, 1984.

[2] T. Caraballo, J. Real, Navier-Stokes equations with delays, R. Soc. Lond. Proc. Ser. A Math. Phys. Eng. Sci., 457 (2001), 2441-2453.

[3] T. Caraballo, J. Real, Asymptotic behaviour of two-dimensional Navier-Stokes equations with delays, R. Soc. Lond. Proc. Ser. A Math. Phys. Eng. Sci., 459 (2003), 3181-3194.

[4] T. Caraballo, J. Real, Attractors for 2D Navier-Stokes models with delays, J. Differential Equations, 205 (2004), 270-296.

[5] T. Caraballo, J. Real, P. E. Kloeden, Unique strong solutions and $V$-attractors of a three dimensional system of globally modified Navier-Stokes equations, Adv. Nonlinear Study, 6 (2006), 411-436.

[6] T. Caraballo, J. Real, P. E. Kloeden, Addendum to the paper "Unique strong solutions and $V$ attractors of a 3-dimensional system of globally modified Navier-Stokes equations, Advanced Nonlinear Studies 6 (2006), 411-436. Adv. Nonlinear Study, 10 (2006), 245-247.

[7] T. Caraballo, P. Marín-Rubio, J. Valero, Attractors for differential equations with unbounded delays, J. Differential Equations, 239 (2007), 311-342.

[8] T. Caraballo, P. E. Kloeden, J. Real, Invariant measures and statistical solutions of the globally modified Navier-Stokes equations, Discrete Cont. Dyn. Syst.-B, 10 (2008), 761-781.

[9] T. Caraballo, J. Real, A. M. Márquez-Durán, Three-dimensional system of globally modified NavierStokes equations with delay, Inter. J. Bifur. Chaos, 20 (2010), 2869-2883.

[10] A. N. Carvalho, J. A. Langa, J. C. Robinson, Attractors of Infinite Dimensional Nonautonomous Dynamical Systems, Applied Mathematical Sciences Vol. 182, Springer, New York, 2013.

[11] M. Chekroun, N. E. Glatt-Holtz, Invariant measures for dissipative dynamical systems: Abstract results and applications, Comm. Math. Phys., 316 (2012), 723-761.

[12] B. Dong, J. Song, Globally regularity and asymptotic behavior of modified Navier-Stokes equations with fractional dissipation, Discrete Cont. Dyn. Syst., 32 (2012), 57-79.

[13] C. Foias, O. Manley, R. Rosa, R. Temam, Navier-Stokes Equations and Turbulence, Cambridge University Press, Cambridge, 2001.

[14] M. J. Garrido-Atienza, P. Marín-Rubio, Navier-Stokes equations with delays on unbounded domains, Nonlinear Anal. 64 (2006), 1100-1118.

[15] J. García-Luengo, P. Marín-Rubio, J. Real, Pullback attractors in $V$ for non-autonomous 2D-NavierStokes equations and their tempered behavior, J. Differential Equations, 252(2012), 4333-4356.

[16] S. M. Guzzo, G. Planas, Existence of solutions for a class of Navier-Stokes equations with infinite delay, Appl. Analysis, 94 (2015), 840-855.

[17] P. E. Kloeden, J. A. Langa, J. Real, Pullback $V$-attractors of a three dimensional system of nonautonomous globally modified Navier-Stokes equations: Existence and finite fractal dimension, Comm. Pure Appl. Anal., 6 (2007), 937-955.

[18] P. E. Kloeden, P. Marín-Rubio, J. Real, Equivalence of invariant measures and stationary statistical solutions for the autonomous globally modified Navier-Stokes equations, Comm. Pure Appl. Anal., 8 (2009), 785-802.

[19] P. E. Kloeden, J. Valero, The weak connectedness of the attainability set of weak solutions of the 3D Navier-Stokes equations, Proc. Roy. Soc. London A, 463 (2007), 1491-1508. 
[20] X. Li, W. Shen, C. Sun, Invariant measures for complex-valued dissipative dynamical systems and applications, Discrete Cont. Dyn. Syst.-B, 22 (2017), 2427-2446.

[21] L. Liu, T. Caraballo, P. Marín-Rubio, Stability results for 2D Navier-Stokes equations with unbounded delay, J. Differential Equations, 265 (2018), 5685-5708.

[22] G. Łukaszewicz, Pullback attractors and statistical solutions for 2-D Navier-Stokes equations, Discrete Cont. Dyn. Syst.-B, 9 (2008), 643-659.

[23] G. Łukaszewicz, J. Real, J. C. Robinson, Invariant measures for dissipative dynamical systems and generalised Banach limits, J. Dyn. Differential Equations, 23 (2011), 225-250.

[24] G. Łukaszewicz, J. C. Robinson, Invariant measures for non-autonomous dissipative dynamical systems, Discrete Cont. Dyn. Syst., 34 (2014), 4211-4222.

[25] P. Marín-Rubio, J. Real, Attractors for 2D-Navier-Stokes equations with delays on some unbounded domains, Nonlinear Anal., 67 (2007), 2784-2799.

[26] P. Marín-Rubio, A. M. Márquez-Durán, J. Real, Three dimensional system of globally modified Navier-Stokes equations with infinite delays, Discrete Cont. Dyn. Syst.-B, 14 (2010), 655-673.

[27] P. Marín-Rubio, A. M. Márquez-Durán, J. Real, Pullback attractors for globally modified NavierStokes equations with infinite delays, Discrete Cont. Dyn. Syst., 31 (2011), 779-796.

[28] P. Marín-Rubio, J. Real, J. Valero, Pullback attractors for a two-dimensional Navier-Stokes model in an infinite delay case, Nonlinear Anal., 74 (2011), 2012-2030.

[29] P. Marín-Rubio, J. Real, A. M. Márquez-Durán, On the convergence of solutions of globally modified Navier-Stokes equations with delays to solutions of Navier-Stokes equations with delays, $A d v$. Nonlinear Study, 11 (2011), 917-927.

[30] P. Marín-Rubio, A. M. Márquez-Durán, J. Real, Asymptotic behavior of solutions for a three dimensional system of globally modified Navier-Stokes equations with a locally Lipschitz delay term, Nonlinear Anal., 79 (2013), 68-79.

[31] A. M. Márquez-Durán, Existence and uniqueness of solutions, and pullback attractor for a system of globally modified 3D-Navier-Stokes equations with finite delay, SeMA Journal, 51 (2010), 117-124.

[32] C. B. G. Moussa, Invariant measures for multivalued semigroups, J. Math. Anal. Appl., 455 (2017), 1234-1248.

[33] M. Romito, The uniqueness of weak solutions of the globally modified Navier-Stokes equations, Adv. Nonlinear Study, 9 (2009), 425-427.

[34] G. R. Sell and Y. You, Dynamics of Evolutionary Equations. Springer, 2002.

[35] R. Temam, Navier-Stokes equations, Theory and Numerical Analysis, 2nd ed., North Holland, Amsterdam, 1979.

[36] X. Wang, Upper-semicontinuity of stationary statistical properties of dissipative systems, Discrete Cont. Dyn. Syst., 23 (2009), 521-540.

[37] C. Zhao, L. Yang, Pullback attractor and invariant measures for the globally modified Navier-Stokes equations, Comm. Math. Sci., 15 (2017), 1565-1580.

[38] C. Zhao, G. Xue, G. Łukaszewicz, Pulback attractor and invariant measures for discrete KleinGordon-Schrödinger equations, Discrete Cont. Dyn. Syst.-B, 23 (2018), 4021-4044.

[39] C. Zhao, T. Caraballo, Asymptotic regularity of trajectory attractor and trajectory statistical solution for the 3D globally modified Navier-Stokes equations, J. Differential Equations, 266 (2019), 72057229. 
[40] C. Zhao, Z. Song, T. Caraballo, Strong trajectory statistical solutions and Liouville type equations for dissipative Euler equations, Appl. Math. Lett., 99 (2020), 105981.

[41] C. Zhao, Y. Li, Z. Song, Trajectory statistical solutions for the 3D Navier-Stokes equations: The trajectory attractor approach, Nonlinear Anal.-RWA, 53(2020), 103077.

[42] C. Zhao, Y. Li, T. Caraballo, Trajectory statistical solutions and Liouville type equations for evolution equations: Abstract results and applications, J. Differential Equations, https://doi.org/10.1016/j. jde.2019.12.011.

[43] C. Zhao, Y. Li, Y. Sang, Using trajectory attractor to construct trajectory statistical solutions for 3D incompressible micropolar flows, Z. Angew. Math. Mech., https://doi.org/10.1002/zamm.201800197.

[44] Z. Zhu, C. Zhao, Pullback attractor and invariant measures for the three-dimensional regularized MHD equations, Discrete Cont. Dyn. Syst., 38(2018), 1461-1477. 
Article ID: CNSNS-D-20-00637

Title: Invariant measures for the 3D globally modified Navier-Stokes equations with unbounded variable delays

Authors: Jintao Wang, Caidi Zhao and Tomás Caraballo

Dear Editor and reviewers,

Thank you very much for considering our manuscript for your kind email. We also want to express our thanks to the referees for their pertinent and detailed suggestions. We have carefully revised the corresponding parts in our manuscript according to the suggestions given by the referees.

Corresponding to the items pointed out by the referee, we make the following revisions:

1) Go through the calculation once again without looking at the expression$\mathrm{s}$ shown in this paper, especially in some long mathematical equations and expressions, such as (4.9), (4.13), etc.

Answer: We calculate (4.9) again and find that the notation $\sigma$ in the expressions (4.7)-(4.9) should all be replaced by $\theta$. The $\sigma$ is changed to $\theta$ therein. We also estimate (4.13) again. There is no mistakes.

2) Page 2 Line 5: "with smooth boundary $\partial \Omega$ " is changed to "with the smooth boundary $\partial \Omega$ ".

3) Page 2 Line 6: "was firstly formulated" is changed to "was formulated"

4) We add some related references as follows:

[11] S. G. Chefranov, A. S. Chefranov, The new analytical solution of the 3D Navier-Stokes equation for compressible medium clarifies the sixth Millennium Prize problem, Cardiometry, (2017), 18-33.

[12] S. G. Chefranov, A. S. Chefranov, The new exact solution of the 
compressible 3D Navier-Stokes equations, Communications in Nonlinear Science and Numerical Simulation, 83(2020), 105118.

[14] S. Das, P. Das, P. Das, Dynamics and control of multidrug-resistant bacterial infection in hospital with multiple delays, Communications in Nonlinear Science and Numerical Simulation, 89(2020), 105279.

[17] R. Garrappa, E. Kaslik, On initial conditions for fractional delay differential equations, Communications in Nonlinear Science and $\mathrm{Nu}$ merical Simulation, 90(2020), 105359.

At the same time, we make some revisions including the English spelling and expression, and revise the bibliography entries accordingly. In addition, all the three authors of this paper agree to transfer the corresponding author to the second author Caidi Zhao. Thank you again for your time and efforts on this manuscript.

Best wishes to you all!

Corresponding author: Caidi Zhao (zhaocaidi2013@163.com)

Department of Mathematics, Wenzhou University, Wenzhou, Zhejiang Province, 325035, People's Republic of China

July 7, 2020 


\title{
Invariant measures for the 3D globally modified Navier-Stokes equations with unbounded variable delays
}

\author{
Jintao Wang ${ }^{a *}, \quad$ Caidi Zhao ${ }^{b \dagger}, \quad$ Tomás Caraballo ${ }^{c \ddagger}$ \\ ${ }^{a}$ Center for Mathematical Sciences, Huazhong University of Science and Technology, \\ Wuhan, Hubei Province, 430074, People's Republic of China \\ ${ }^{b}$ Department of Mathematics, Wenzhou University, \\ Wenzhou, Zhejiang Province, 325035, People's Republic of China \\ ${ }^{c}$ Departmento de Ecuaciones Diferenciales y Análisis Numérico, Facultad de Matemáticas, \\ Universidad de Sevilla, c/Tarfia s/n, 41012-Sevilla, Spain
}

July 7,2020

\begin{abstract}
This article investigates the three-dimensional globally modified Navier-Stokes equations with unbounded variable delays. Firstly, we prove the global well-posedness of the solutions, and give the existence of the pullback attractor for the associated process. Then, we construct a family of invariant Borel probability measures, which is supported by the pullback attractor.
\end{abstract}

Keywords: Globally modified Navier-Stokes equations; Unbounded variable delays; Invariant measures; Pullback attractors.

MSC2010: 35B41, 34D35, 76F20

\section{Introduction}

In this article, we study the following three-dimensional (3D) system of globally modified NavierStokes (GMNS for short) equations with unbounded variable delays

$$
\begin{cases}\frac{\partial u}{\partial t}-\nu \Delta u+F_{N}\left(\|u\|_{V}\right)[(u \cdot \nabla) u]+\nabla p=f(x, t)+g(t, u(t-\rho(t))) & \text { in }(\tau,+\infty) \times \Omega, \\ \nabla \cdot u=0 & \text { in }(\tau,+\infty) \times \Omega, \\ u=0 & \text { on }(\tau,+\infty) \times \partial \Omega, \\ u(\tau+s, x)=\phi(s, x) & \text { in }(-\infty, 0] \times \Omega,\end{cases}
$$

where the unknown functions $u=u(x, t)$ and $p=p(x, t)$ denote, respectively, the velocity field of the fluid and the pressure, the positive constant $\nu$ is the viscosity coefficient, $f(x, t)$ is the density of the

\footnotetext{
${ }^{*}$ E-mail: wangjt@hust.edu.cn

${ }^{\dagger}$ Corresponding author, E-mail: zhaocaidi2013@163.com or zhaocaidi@wzu.edu.cn

${ }^{\ddagger}$ E-mail: caraball@us.es
} 
volume forces, $g$ is the external force affected by memory during the time range $(-\infty, \tau], \rho: \mathbb{R} \rightarrow \mathbb{R}^{+}$ is a delay function satisfying $\rho \in L_{\mathrm{loc}}^{\infty}(\mathbb{R})$ and $\phi$ is the initial datum on the interval $(-\infty, 0]$. Here $\|u\|_{V}=\|\nabla u\|_{\left(L^{2}(\Omega)\right)^{3}}$ and the function $F_{N}(\cdot):(0,+\infty) \mapsto(0,1]$ is defined as

$$
F_{N}(r):=\min \{1, N / r\}, \quad r \in(0,+\infty),
$$

where $N$ is a positive integer. In addition, $\Omega \subset \mathbb{R}^{3}$ is an open bounded set with the smooth boundary $\partial \Omega$.

The GMNS equations with $g \equiv 0$ (that is without delays) was formulated in [5]. It is one kind of global modifications of the Navier-Stokes (NS for short) equations with the modifying factor $F_{N}\left(\|u\|_{V}\right)$ depending on the $\left(L^{2}(\Omega)\right)^{3}$-norm of $\nabla u$. This modification brings some good properties to the solutions: global existence, uniqueness and regularity, in comparison with the multiple-solution property of NS equations (see $[11,12,16]$ and the references therein for readers' perspective). The GMNS equations are useful in obtaining some new results for the 3D NS equations. For instance, it was used in [5] to establish the existence of bounded entire weak solutions for the 3D NS equations, and in [23], the authors applied it to show weak compactness and weak connectedness of the attainability set of the weak solutions of the 3D NS equations satisfying an energy inequality. At the same time, it was proved in $[5,33]$ that the solutions of the GMNS equations converge to those of the 3D NS equations in some proper sense. Therefore, the GMNS equations (1.1) with $g \equiv 0$ can be regarded as an approximation to the 3D NS equations.

The GMNS equations (1.1) with $g \equiv 0$ have been extensively studied. For example, one can see $[5,6,15,21,37]$ for the existence and uniqueness of the weak and strong solutions, $[8,22,28,41]$ for invariant measure and statistical solutions, [43] for the existence of trajectory attractor and trajectory statistical solutions. However, the real world is inundated with many situations in which the model is better described with some terms containing delays appearing in the equations. These delays may appear, for instance, when the current behavior is influenced by its previous states, or one wants to control the system by imposing an external force which depends upon both the present state and the history of the solutions. The delayed partial differential equations are usually used to describe these delayed phenomena ( $[14,17])$.

Nowadays, delayed partial differential equations have drawn much attention and have been extensively studied. For example, the existence and asymptotic behaviors of solutions for the NS equations with finite delays were investigated in $[2-4,18,29]$; the existence of pullback attractors for the NS equations with finite or infinite delays was studied in [7,20,25,32]. Some known references concerning the delayed 3D GMNS equations are summarized as:

$1^{\circ}$ Finite delays: the existence, uniqueness and global asymptotic exponentially stability of the stationary solution, as well as the convergence of solutions to those of the NS equations were established in $[33,34]$.

$2^{\circ}$ Infinite delays: the well-posedness and global exponential decay of the solutions to the stationary solution, as well as the existence of pullback attractor were investigated in [30,31].

$3^{\circ}$ Bounded variable delays: the existence and uniqueness of solutions, as well as the existence of pullback attractors were proved in $[9,35]$.

However, up to our knowledge, there is no reference concerning the 3D GMNS equations with unbounded variable delays. 
The motivation of this article is to investigate the existence of invariant Borel probability measures for the 3D GMNS equations with unbounded variable delays. The invariant measures and statistical solutions have played a significant role and developed into a crucial tool in the research of turbulence (see Foias et al. [16]) in recent decades. This is mainly due to the fact that some time-average quantities essentially measure several important aspects of turbulent flows. The invariant measures and statistical properties of dissipative systems were studied by a number of researchers after that. For instance, Wang considered the upper semi-continuity of stationary statistical properties for dissipative systems in [40]. Łukaszewicz, Real and Robinson in [27] constructed the invariant measures for general continuous dynamical systems on metric spaces by using the generalized Banach limit. For a much wider class of dissipative semigroups, Chekroun and Glatt-Holtz [13] also applied the generalized Banach limit to constructing the invariant measures, but they generalized and simplified the proofs of [27] and [40].

Recently, a series of works developed some techniques to provide a construction of invariant measures for non-autonomous systems with minimal assumptions on the underlying dynamical process (see Foias et al. [16], Wang [40] and Łukaszewicz et al. [26-28]). Nowadays, these theories have been employed to establish invariant measures for some evolution equations, (see e.g. [24, 36, 41, 48] and the references therein). However, as far as we know, there is no reference investigating the invariant measures for delayed evolution equations.

The main result of the current article is the existence of invariant Borel probability measures for the 3D GMNS equations with unbounded variable delays. We will use the abstract theory for dissipative non-autonomous system in [28, Theorem 3.1]. To this end, we shall prove that the solution operators associated to the problem (1.1) generate a continuous process $\{U(t, \tau)\}_{t \geqslant \tau}$ in the phase space $\mathcal{C}_{\gamma}(H)$ (see notation in Section 2) and

(1) the process $\{U(t, \tau)\}_{t \geqslant \tau}$ is pullback strongly bounded in $\mathcal{C}_{\gamma}(H)$;

(2) the process $\{U(t, \tau)\}_{t \geqslant \tau}$ is pullback asymptotically compact in $\mathcal{C}_{\gamma}(H)$;

(3) for each given $t_{*} \in \mathbb{R}$ and $\phi_{*} \in \mathcal{C}_{\gamma}(H)$, the $\mathcal{C}_{\gamma}(H)$-valued function $\tau \mapsto U\left(t_{*}, \tau\right) \phi_{*}$ is continuous and bounded on $(-\infty, t]$ (this is called $\tau$-continuity of the process $\{U(t, \tau)\}_{t \geqslant \tau}$ ).

We first use a Galerkin approximation argument, and some a priori estimates, to prove the global well-posedness of the solutions to the problem (1.1). Also we do some estimates of the solutions to obtain the existence of strongly bounded pullback absorbing sets. Secondly we apply the Ascoli-Arzelà type theorem ( [1]) to establish the pullback asymptotic compactness of the process $\{U(t, \tau)\}_{t \geqslant \tau}$. This is prompted by the delay appearing in the equations. Since the problem discussed contains unbounded variable delays, it seems suitable for us to choose $C_{\gamma}(H)$ as the phase space. It has been shown that the Ascoli-Arzelà theorem is a powerful tool to investigate the compactness of subsets of $C_{\gamma}(H)$. Thirdly, for each given $t_{*} \in \mathbb{R}$ and $\phi_{*} \in C_{\gamma}(H)$, the continuity of the $C_{\gamma}(H)$-valued function $\tau \mapsto U\left(t_{*}, \tau\right) \phi_{*}$ on $(-\infty, t]$ is not a clear fact (see Lemma 4.4 and the auxiliary lemmas). There are some essential differences between the autonomous dynamical system and non-autonomous one, say the continuous dependence of the dynamical system on their parameters. Even if we have known that the $C_{\gamma}(H)$ valued function $t \mapsto U(t, \tau) \phi_{*}$ is continuous on $\mathbb{R}$ for every given $\tau$ and $\phi_{*} \in C_{\gamma}(H)$, the convergence $\left\|U\left(t_{*}, \tau\right) \phi_{*}-\phi_{*}\right\|_{\gamma} \rightarrow 0$ as $\tau \rightarrow t_{*}^{-}$may still depend on $\tau$ itself. In fact, when $\tau \rightarrow t_{*}^{-}, U\left(t_{*}, \tau\right) \phi_{*}$ also changes with different initial times $\tau$. This is caused naturally by the non-autonomous and delayed phenomena. We will take advantage of the structure of the GMNS equations to prove the $\tau$-continuity of 
the process $\{U(t, \tau)\}_{t \geqslant \tau}$.

This article is organized as follows. In the next section, we first introduce some notations and operators, and then prove the global well-posedness of solutions to the problem (1.1). Section 3 is devoted to the proof of the existence of pullback attractors. In Section 4, we first prove the $\tau$-continuity of the process and then construct a family of invariant Borel probability measures which is supported by the pullback attractors. We end the article with conclusions and remarks in Section 5.

\section{Global well-posedness of solutions}

In this section, we first introduce some notations and operators. Then we prove the global wellposedness of the problem (1.1).

In this article, we use the following notations:

$\mathbb{L}^{2}(\Omega)=\left(L^{2}(\Omega)\right)^{3}$-the 3D Lebesgue space with norm $\|\cdot\|_{\mathbb{L}^{2}(\Omega)}=\|\cdot\|$;

$\mathbb{H}^{1}(\Omega)=$ the $3 \mathrm{D}$ Sobolev space $\left\{\phi \in \mathbb{L}^{2}(\Omega) \mid \nabla \phi \in \mathbb{L}^{2}(\Omega)\right\}$ with norm $\|\nabla \cdot\|_{\mathbb{L}^{2}(\Omega)}$;

$\mathbb{H}_{0}^{1}(\Omega)=$ closure of $\left(\mathcal{C}_{0}^{\infty}(\Omega)\right)^{3}$ in $\mathbb{H}^{1}(\Omega)$ with norm $\|\cdot\|_{\mathbb{H}^{1}(\Omega)}$;

$\mathcal{V}=\left\{\phi \in\left(\mathcal{C}_{0}^{\infty}(\Omega)\right)^{3} \mid \nabla \cdot \phi=0\right\}$

$H=$ closure of $\mathcal{V}$ in $\mathbb{L}^{2}(\Omega)$ with norm $\|\cdot\|$ and inner product $(\cdot, \cdot)$;

$V=$ closure of $\mathcal{V}$ in $\mathbb{H}^{1}(\Omega)$ with norm $\|\cdot\|_{V}=\|\nabla \cdot\|_{\mathbb{L}^{2}(\Omega)}$;

$V^{\prime}=$ dual space of $V$ with norm $\|\cdot\|_{V^{\prime}} ;\langle\cdot, \cdot\rangle-$ the dual pairing between $V$ and $V^{\prime}$;

In addition, we will use the notation $a \lesssim b$ to mean that $a \leqslant c b$ for a universal constant $c>0$ that only depends on the parameters coming from the problem and will not result in confusion.

For short, we will write the problem (1.1) in a functional form. To this end, we introduce some classical operators which are usually used to handle the 3D incompressible NS and GMNS equations. At the same time, we will select some known estimates and properties (see e.g. [39]) of these operators.

We first consider the operator $A: V \rightarrow V^{\prime}$ defined as

$$
\langle A u, v\rangle=(\nabla u, \nabla v), \quad \forall u, v \in V,
$$

where $D(A)=\mathbb{H}^{2}(\Omega) \cap V$ and $\mathbb{H}^{2}(\Omega)=\left\{\phi \in \mathbb{H}^{1}(\Omega) \mid \Delta \phi \in \mathbb{L}^{2}(\Omega)\right\}$. In fact, for each $u \in D(A)$, $A u=-P \Delta u$, and hence $A$ is the Stokes operator, where $P$ is the Leray-Helmholtz projection from $\mathbb{L}^{2}(\Omega)$ onto $H$.

Secondly, we define a trilinear form $b(\cdot, \cdot, \cdot)$ as follows,

$$
b(u, v, w)=\sum_{i, j=1}^{3} \int_{\Omega} u_{i} \frac{\partial v_{j}}{\partial x_{i}} w_{j} \mathrm{~d} x, \quad \forall u, v, w \in \mathbb{H}_{0}^{1}(\Omega) .
$$

Notice that $V$ is a closed subspace of $\mathbb{H}_{0}^{1}(\Omega)$. One can check that $b(u, v, w)$ is continuous on $V \times V \times V$, and $b(u, v, w)=-b(u, w, v), b(u, v, v)=0, \forall u, v, w \in V$. For each $u, v \in V$,

$$
\langle B(u, v), w\rangle=b(u, v, w), \quad \forall w \in V,
$$

defines a continuous function $B(u, v)$ on $V \times V$. At the same time, we have (cf. [39])

$$
\begin{array}{ll}
\|b(u, v, w)\| \lesssim\|u\|_{V}^{1 / 2}\|A u\|^{1 / 2}\|v\|_{V}\|w\|, & \forall u \in D(A), v \in V, w \in H \\
\|b(u, v, w)\| \lesssim\|u\|_{V}\|v\|_{V}\|w\|_{V}^{1 / 2}\|w\|^{1 / 2}, & \forall u, v, w \in V .
\end{array}
$$


We further set

$$
\begin{aligned}
& b_{N}(u, v, w)=F_{N}\left(\|v\|_{V}\right) b(u, v, w), \quad \forall u, v, w \in V, \\
& \left\langle B_{N}(u, v), w\right\rangle=b_{N}(u, v, w), \quad \forall u, v, w \in V .
\end{aligned}
$$

Then $b_{N}(u, v, w)$ is linear in $u$ and $w$, but not linear in $v$. However, we still have (see [37])

$$
\begin{aligned}
b_{N}(u, v, v) & =0, \quad \forall u, v \in V, \\
\left|b_{N}(u, v, w)\right| & \lesssim N\|u\|_{V}\|w\|_{V}, \quad \forall u, v, w \in V, \\
\left|b_{N}(u, v, w)\right| & \lesssim N\|A u\|\|w\|, \quad \forall u \in D(A), v \in V, w \in H .
\end{aligned}
$$

Furthermore, for every $u, v \in V$ and each $N>0$,

$$
\begin{aligned}
0 & \leqslant\|u\|_{V} F_{N}\left(\|u\|_{V}\right) \leqslant N \\
\left|F_{N}\left(\|u\|_{V}\right)-F_{N}\left(\|v\|_{V}\right)\right| & \leqslant \frac{1}{N} F_{N}\left(\|u\|_{V}\right) F\left(\|v\|_{V}\right)\|u-v\|_{V} .
\end{aligned}
$$

To formulate the problem (1.1) in a proper manner, we require that the functions $\rho(t), f(x, t)$ and $g(t, u(t-\rho(t)))$ in $(1.1)$ satisfy some natural hypotheses.

(H1) Suppose that the function $\rho: \mathbb{R} \mapsto \mathbb{R}_{+}$belongs to $L_{\mathrm{loc}}^{\infty}(\mathbb{R})$ and $f(t, x) \in L_{\mathrm{loc}}^{2}\left(\mathbb{R} ; \mathbb{L}^{2}(\Omega)\right)$. Also assume that $g: \mathbb{R} \times H \rightarrow H$ satisfies

(g1) for every $u \in H$, the mapping $\mathbb{R} \ni t \mapsto g(t, u)$ is measurable;

(g2) $g(t, 0)=0$ for all $t \in \mathbb{R}$;

(g3) there exists a positive constant $\gamma$ and a nonnegative function $L_{g}: \mathbb{R} \rightarrow \mathbb{R}_{+}$such that

$$
L_{g}(\cdot) e^{\gamma \rho(\cdot)} \in L_{\mathrm{loc}}^{2}(\mathbb{R}) \quad \text { and } \quad\|g(t, u)-g(t, v)\| \leqslant L_{g}(t)\|u-v\|, \quad \forall t \in \mathbb{R}, \forall u, v \in H .
$$

Remark 2.1. In Remark 3.1 we will give a concrete example to show the existence of the functions $\rho(t)$, $f(t, x), g(t, u), L_{g}(t)$ and the constant $\gamma$ that satisfy our assumption (H1). In the sequel, the constant $\gamma$ is from $(g 3)$.

In order to deal with the unbounded variable delays, we consider the space

$$
C_{\gamma}(H):=\left\{\phi \in C((-\infty, 0] ; H): \sup _{s \leqslant 0} e^{\gamma s}\|\phi(s)\|<+\infty, \exists \lim _{s \rightarrow-\infty} e^{\gamma s} \phi(s) \in H\right\},
$$

with the norm $\|\phi\|_{\gamma}:=\sup _{s \leqslant 0} e^{\gamma s}\|\phi(s)\|$. Then $\left(C_{\gamma}(H),\|\cdot\|_{\gamma}\right)$ is a Banach space. In addition, for each $\tau \in \mathbb{R}$ we denote conventionally $u_{\tau}(s)=u(\tau+s)$ with $s \in(-\infty, 0]$.

We now specify the definition of the weak solution to the problem (1.1).

Definition 2.1. Let $\tau \in \mathbb{R}$ and $\phi \in C_{\gamma}(H)$ be given. If for all $T>\tau$, a function $u \in C((-\infty, T], H) \cap$ $L^{2}(\tau, T ; V)$ with $u_{\tau}=\phi$ satisfies

$$
\frac{\mathrm{d} u(t)}{\mathrm{d} t}+\nu A u(t)+B_{N}(u(t), u(t))=f(t)+g(t, u(t-\rho(t)),
$$

in the sense of distribution $\mathcal{D}^{\prime}\left(\tau,+\infty ; V^{\prime}\right)$, then we call $u$ a weak solution of the problem (1.1) corresponding to the initial datum $\phi$. 
For the existence and uniqueness of solutions to the problem (1.1), we have the following result.

Theorem 2.1. Let the assumption (H1) hold true. Then for each $\tau \in \mathbb{R}$ and $\phi \in C_{\gamma}(H)$, there corresponds a unique weak solution $u(\cdot)=u(\cdot ; \tau, \phi)$ to the problem (1.1), and $u$ is in fact a strong solution in the sense that

$$
u \in C((\tau, T], V) \cap L^{2}(\tau+\varepsilon, T ; D(A)), \quad \forall \varepsilon>0, \quad \forall T>\tau+\varepsilon .
$$

Moreover, if $\phi(0) \in V$, then

$$
u \in C([\tau, T] ; V) \cap L^{2}(\tau, T ; D(A)), \quad \forall T>\tau .
$$

Proof. The proof is similar to that of [30, Theorem 2] and [31, Theorem 1] with the unbounded variable delays in place of the infinite delays. Following the proof of [30, Theorem 2] with some different estimates, we present the parts that will be used in the sequel of this article.

For the Galerkin approximation solution $u^{(m)}$, we have

$$
\begin{aligned}
\frac{\mathrm{d}}{\mathrm{d} t}\left\|u^{(m)}(t)\right\|^{2}+2 \nu\left\|u^{(m)}(t)\right\|_{V}^{2} & =2\left(f(t), u^{(m)}(t)\right)+2\left(g\left(t, u^{(m)}(t-\rho(t))\right), u^{(m)}(t)\right) \\
& \lesssim \nu\left\|u^{(m)}(t)\right\|_{V}^{2}+\|f(t)\|^{2}+2 L_{g}(t)\left\|u^{(m)}(t-\rho(t))\right\|\left\|u^{(m)}(t)\right\| \\
& \lesssim \nu\left\|u^{(m)}(t)\right\|_{V}^{2}+\|f(t)\|^{2}+2 L_{g}(t) e^{\gamma \rho(t)}\left\|u_{t}^{m}\right\|_{\gamma}^{2},
\end{aligned}
$$

using the facts that $\left\|u^{(m)}(t)\right\|=\left\|u_{t}^{(m)}(0)\right\| \leqslant\left\|u_{t}^{(m)}\right\|_{\gamma}$ and

$$
\left\|u^{(m)}(t-\rho(t))\right\|=e^{\gamma \rho(t)} e^{-\gamma \rho(t)}\left\|u^{(m)}(t-\rho(t))\right\| \leqslant e^{\gamma \rho(t)}\left\|u_{t}^{(m)}\right\|_{\gamma} .
$$

Hence, for all $t \geqslant \tau$ there holds

$$
\left\|u^{(m)}(t)\right\|^{2}+\nu \int_{\tau}^{t}\left\|u^{(m)}(s)\right\|_{V}^{2} \mathrm{~d} s \lesssim\left\|u^{(m)}(\tau)\right\|^{2}+\int_{\tau}^{t}\left(\|f(s)\|^{2}+2 L_{g}(s) e^{\gamma \rho(s)}\left\|u_{s}^{(m)}\right\|_{\gamma}^{2}\right) \mathrm{d} s .
$$

By (2.13) and the definition of the norm $\left\|u_{t}^{(m)}\right\|_{\gamma}^{2}$, we have

$$
\begin{aligned}
\left\|u_{t}^{(m)}\right\|_{\gamma}^{2} & \lesssim \max \left\{\sup _{\theta \leqslant \tau-t} e^{2 \gamma \theta}\|\phi(\theta+t-\tau)\|^{2}\right. \\
& \lesssim \max \left\{\sup _{\theta \in[\tau-t, 0]}\left(e^{2 \gamma \theta}\|u(\tau)\|^{2}+e^{2 \gamma \theta} \int_{\tau \leqslant 0}^{2 \gamma(\theta+\tau-t)}\|\phi(\theta)\|^{2},\|u(\tau)\|^{2}+\int_{\tau}^{t}\left(\|f(s)\|^{2}+2 L_{g}(s) e^{\gamma \rho(s)}\left\|u_{s}^{(m)}\right\|_{\gamma}^{2}\right) \mathrm{d} s\right)\right\} \\
& \left.\lesssim\|\phi\|_{\gamma}^{2}+\int_{\tau}^{t}\left(\|f(s)\|^{2}+2 L_{g}(s) e^{\gamma \rho(s)}\left\|u_{s}^{(m)}\right\|_{\gamma}^{2}\right) \mathrm{d} s\right\}
\end{aligned}
$$

Applying Gronwall's lemma to (2.14) gives

$$
\left\|u_{t}^{(m)}\right\|_{\gamma}^{2} \lesssim\left(\|\phi\|_{\gamma}^{2}+\int_{\tau}^{t}\|f(s)\|^{2} \mathrm{~d} s\right) \exp \left(2 \int_{\tau}^{t} L_{g}(s) e^{\gamma \rho(s)} \mathrm{d} s\right)
$$


We then conclude from (2.13) and (2.15) that, with the assumption (H1) in hand,

$$
\begin{aligned}
& \left\|u_{t}^{(m)}\right\|_{\gamma}^{2}+\left\|u^{(m)}\right\|_{L^{2}(\tau, T ; V)}^{2} \lesssim 1, \quad \forall t \in[\tau, T], \forall\|\phi\|_{\gamma} \leqslant R, \forall m \geqslant 1, \\
& \int_{\tau}^{T}\left\|g\left(s, u^{(m)}(s-\rho(s))\right)\right\|^{2} \mathrm{~d} s \leqslant \int_{\tau}^{T}\left(L_{g}(s) e^{\gamma \rho(s)}\right)^{2}\left\|u_{s}^{(m)}\right\|_{\gamma}^{2} \mathrm{~d} s \lesssim\left\|L_{g} e^{\gamma \rho}\right\|_{L^{2}(\tau, T)}^{2} .
\end{aligned}
$$

Estimate (2.16) implies that

$$
g\left(\cdot, u^{(m)}(\cdot-\rho(\cdot))\right) \in L^{2}\left(\tau, T ; \mathbb{L}^{2}(\Omega)\right) .
$$

The rest tasks are to prove that the Galerkin approximate solutions $\left\{u^{(m)}\right\}$ possess a convergent subsequence and its limiting function is the solution satisfying Theorem 2.1. The procedures are similar to those of [30, Theorem 2] and we omit the details here.

The result below shows that the solutions to (1.1) depend continuously on the initial data.

Theorem 2.2. Let the assumption (H1) hold. Let $u=u(\cdot ; \tau, \phi)$ and $\tilde{u}=\tilde{u}(\cdot ; \tau, \tilde{\phi})$ be two solutions of the problem (1.1) with the initial data $\phi$ and $\tilde{\phi}$ at the initial time $\tau$, respectively. Then we have

$$
\begin{aligned}
& \max _{\theta \in[\tau, t]}\|u(\theta)-\tilde{u}(\theta)\|^{2} \\
\lesssim & \|\phi(0)-\tilde{\phi}(0)\|^{2} \exp \left(\int_{\tau}^{t} 2 L_{g}(s) e^{\gamma \rho(s)} \mathrm{d} s+(t-\tau)\right) \\
& +\|\phi-\tilde{\phi}\|_{\gamma}^{2} \int_{\tau}^{t} L_{g}(s) e^{\gamma \rho(s)} \exp \left(\int_{\tau}^{s} 2 L_{g}(\theta) e^{\gamma \rho(\theta)} \mathrm{d} \theta+(s-\tau)\right) \mathrm{d} s .
\end{aligned}
$$

The proof of Theorem 2.2 is analogous to that of [31, Proposition 1] and we omit the details.

\section{Existence of the pullback attractors}

From Theorems 2.1 and 2.2 we can conclude that the solution operators $U(t, \tau): C_{\gamma}(H) \rightarrow C_{\gamma}(H)$ defined by

$$
U(t, \tau) \phi(s)=u_{t}(s), \quad \tau \leqslant t, \quad s \in(-\infty, 0],
$$

generate a continuous process $\{U(t, \tau)\}_{t \geqslant \tau}$ on the phase space $C_{\gamma}(H)$, where $u=u(\cdot ; \tau, \phi)$ is the solution of the problem (1.1) corresponding to the initial function $\phi$ at the initial time $\tau$. In this section, we first prove that $\{U(t, \tau)\}_{t \geqslant \tau}$ possesses a bounded pullback absorbing set and is pullback asymptotically compact in $C_{\gamma}(H)$. Then we obtain the existence of the pullback attractor. For related definitions and results concerning the evolution processes and pullback attractors, we can refer to $[4,10,38]$ and the references therein.

In the sequel, we pick $\gamma$ and some parameter $\mu$ such that

$$
0<\mu<\nu \text { and }(\nu-\mu) \lambda_{1}<\gamma
$$

where $\lambda_{1}=\inf _{v \in V \backslash\{0\}} \frac{\|v\|_{V}^{2}}{\|v\|^{2}}>0$ is the first eigenvalue of the Stokes operator $A$. To obtain the existence of the bounded pullback absorbing set, we need another assumption for the functions $L_{g}(\cdot) e^{\gamma \rho(\cdot)}$ and $f$. 
(H2) Assume there is a constant $\mu$ satisfying (3.2) such that

$$
\begin{aligned}
& \underset{t \in \mathbb{R}}{\operatorname{ess} \sup } L_{g}(t) e^{\gamma \rho(t)}<(\nu-\mu) \lambda_{1} \leqslant \gamma, \\
& \int_{-\infty}^{0} e^{\sigma s}\|f(s)\|^{2} \mathrm{~d} s<+\infty
\end{aligned}
$$

hereinafter the constant $\sigma$ is given by

$$
\sigma:=2(\nu-\mu) \lambda_{1}-2 \underset{t \in \mathbb{R}}{\operatorname{ess} \sup } L_{g}(t) e^{\gamma \rho(t)} .
$$

Remark 3.1. We set

$$
\left\{\begin{array}{l}
\rho(t)=|t|, \quad t \in \mathbb{R} \\
g(t, u)=\exp \left(-e^{|t|}\right) u, \quad t \in \mathbb{R}, u \in H \\
L_{g}(t)=\exp \left(-e^{|t|}\right), \quad t \in \mathbb{R} \\
\gamma=e
\end{array}\right.
$$

then the conditions in (H1) are satisfied and

$$
\underset{t \in \mathbb{R}}{\operatorname{ess} \sup _{g}} L_{g}(t) e^{\gamma \rho(t)}=e^{\gamma \ln \gamma-\gamma}=e^{0}=1<e=\gamma .
$$

If we take $1<\nu<e$ and $\mu$ small enough, then (3.3) are satisfied. Thus, the functions and constant presented by (3.6) satisfy the conditions in (H1) and (H2). For the existence of the function $f(x, t)$ satisfying (H1) and (H2), we refer to [42, Example 3.1].

We denote by $\mathcal{P}\left(C_{\gamma}(H)\right)$ the family of all subsets of $C_{\gamma}(H)$ and consider the families of nonempty sets $\widehat{D}=\{D(t): t \in \mathbb{R}\} \subseteq \mathcal{P}\left(C_{\gamma}(H)\right)$. Let $\mathcal{D}_{\sigma}$ be the nonempty class of families $\widehat{D}=\{D(t): t \in$ $\mathbb{R}\} \subseteq \mathcal{P}\left(C_{\gamma}(H)\right)$ parameterized by time $t$ and satisfying

$$
\lim _{\tau \rightarrow-\infty}\left(e^{\sigma \tau} \sup _{v \in D(\tau)}\|v\|_{\gamma}^{2}\right)=0 .
$$

The class $\mathcal{D}_{\sigma}$ defined above is always called a tempered universe.

Lemma 3.1. Let the assumptions (H1) and (H2) hold. Then the process $\{U(t, \tau)\}_{t \geqslant \tau}$ possesses a bounded pullback absorbing set in $C_{\gamma}(H)$.

Proof. Pick some $\mu$ satisfying (3.2), and let $u=u(\cdot ; \tau, \phi)$ be the solution of the problem (1.1) corresponding to the initial function $\phi$ at the initial time $\tau$. Then we can get

$$
\frac{\mathrm{d}}{\mathrm{d} t}\|u(t)\|^{2}+2(\nu-\mu) \lambda_{1}\|u(t)\|^{2}+\mu\|u(t)\|_{V}^{2} \lesssim\|f(t)\|^{2}+2 L_{g}(t) e^{\gamma \rho(t)}\left\|u_{t}\right\|_{\gamma}^{2}, \quad t>\tau .
$$

Hence for all $t \geqslant \tau$,

$$
\begin{aligned}
& \|u(t)\|^{2}+\mu \int_{\tau}^{t} e^{-2(\nu-\mu) \lambda_{1}(t-s)}\|u(s)\|_{V}^{2} \mathrm{~d} s \\
\lesssim & e^{-2(\nu-\mu) \lambda_{1}(t-\tau)}\|u(\tau)\|^{2}+\int_{\tau}^{t} e^{-2(\nu-\mu) \lambda_{1}(t-s)}\left(\|f(s)\|^{2}+2 L_{g}(s) e^{\gamma \rho(s)}\left\|u_{s}\right\|_{\gamma}^{2}\right) \mathrm{d} s .
\end{aligned}
$$


Inequality (3.8) implies that

$$
\begin{aligned}
\left\|u_{t}\right\|_{\gamma}^{2} \lesssim \max \left\{\sup _{\theta \in(-\infty, \tau-t]} e^{2 \gamma \theta}\|\phi(\theta+t-\tau)\|^{2}, \sup _{\theta \in[\tau-t, 0]}\left(e^{2 \gamma \theta-2(\nu-\mu) \lambda_{1}(t+\tau-\theta)}\|u(\tau)\|^{2}\right.\right. \\
\left.\left.+e^{2 \gamma \theta} \int_{\tau}^{t+\theta} e^{-2(\nu-\mu) \lambda_{1}(t+\theta-s)}\left(\mid f(s)\left\|^{2}+2 L_{g}(s) e^{\gamma \rho(s)}\right\| u_{s} \|_{\gamma}^{2}\right) \mathrm{d} s\right)\right\} .
\end{aligned}
$$

By the choice of $\mu$ in (3.2), we have

$$
\begin{aligned}
& \sup _{\theta \in(-\infty, \tau-t]} e^{2 \gamma \theta}\|\phi(\theta+t-\tau)\| \leqslant e^{-(\nu-\mu) \lambda_{1}(t-\tau)}\|\phi\|_{\gamma}, \\
& \sup _{\theta \in[\tau-t, 0]} e^{2 \gamma \theta-2(\nu-\mu) \lambda_{1}(t+\tau-\theta)}\|u(\tau)\|^{2} \leqslant e^{-2(\nu-\mu) \lambda_{1}(t-\tau)}\|u(\tau)\|^{2}, \\
& \sup _{\theta \in[\tau-t, 0]} e^{2 \gamma \theta} \int_{\tau}^{t+\theta} e^{-2(\nu-\mu) \lambda_{1}(t+\theta-s)}\left(\|f(s)\|^{2}+2 L_{g}(s) e^{\gamma \rho(s)}\left\|u_{s}\right\|_{\gamma}^{2}\right) \mathrm{d} s \\
& \leqslant \int_{\tau}^{t} e^{-2(\nu-\mu) \lambda_{1}(t-s)}\left(\|f(s)\|^{2}+2 L_{g}(s) e^{\gamma \rho(s)}\left\|u_{s}\right\|_{\gamma}^{2}\right) \mathrm{d} s .
\end{aligned}
$$

It then follows from (3.9)-(3.12) that

$$
\left\|u_{t}\right\|_{\gamma}^{2} \lesssim e^{-2(\nu-\mu) \lambda_{1}(t-\tau)}\|\phi\|_{\gamma}^{2}+\int_{\tau}^{t} e^{-2(\nu-\mu) \lambda_{1}(t-s)}\left(\|f(s)\|^{2}+2 L_{g}(s) e^{\gamma \rho(s)}\left\|u_{s}\right\|_{\gamma}^{2}\right) \mathrm{d} s .
$$

Applying Gronwall's inequality to (3.13) yields

$$
\begin{aligned}
\left\|u_{t}\right\|_{\gamma}^{2} \lesssim & \|\phi\|_{\gamma}^{2} \exp \left\{-2(\nu-\mu) \lambda_{1}(t-\tau)+2 \int_{\tau}^{t} L_{g}(s) e^{\gamma \rho(s)} \mathrm{d} s\right\} \\
& +\int_{\tau}^{t}\|f(s)\|^{2} \exp \left\{-2(\nu-\mu) \lambda_{1}(t-s)+2 \int_{s}^{t} L_{g}(\sigma) \mathrm{e}^{\gamma \rho(\sigma)} \mathrm{d} \sigma\right\} \mathrm{d} s \\
& \lesssim\|\phi\|_{\gamma}^{2} e^{-\sigma(t-\tau)}+\int_{\tau}^{t}\|f(s)\|^{2} e^{-\sigma(s-\tau)} \mathrm{d} s .
\end{aligned}
$$

Now we define the time-dependent family $\hat{\mathcal{B}}=\{\mathcal{B}(t): t \in \mathbb{R}\} \in \mathcal{P}\left(C_{\gamma}(H)\right)$ as

$$
\mathcal{B}(t)=\left\{v \in C_{\gamma}(H):\|v\|_{\gamma}^{2} \lesssim 1+\int_{-\infty}^{t} e^{-\sigma(t-s)}\|f(s)\|^{2} \mathrm{~d} s\right\}
$$

Then $\hat{\mathcal{B}}$ is the desired pullback $\mathcal{D}_{\sigma}$-absorbing set for $\{U(t, \tau)\}_{t \geqslant \tau}$ in $C_{\gamma}(H)$.

To establish the pullback $\mathcal{D}_{\sigma}$-asymptotic compactness for $\{U(t, \tau)\}_{t \geqslant \tau}$ in $C_{\gamma}(H)$, we will employ the generalized Ascoli-Arzelà type theorem ( [1]) to prove the following auxiliary lemma.

Lemma 3.2. Let $\left\{\tau_{n}\right\}_{n \geqslant 1}$ be a sequence such that $\tau_{n} \rightarrow-\infty$ as $n \rightarrow+\infty$ and $\tau_{n} \leqslant t_{0}$ for some fixed $t_{0} \in \mathbb{R}$, and $\left\{\phi_{n}\right\}_{n \geqslant 1}$ a sequence of functions with $\phi_{n} \in \mathcal{B}\left(\tau_{n}\right)$ for each positive integer $n$. Denote by $u^{(n)}=u^{(n)}\left(\cdot ; \tau_{n}, \phi_{n}\right)$ the solutions of the problem (1.1) corresponding to the initial data $\phi_{n}$ at the initial time $\tau_{n}$. Then there exist a subsequence (still denoted the same) $\left\{u_{t_{0}}^{(n)}\right\}_{n \geqslant 1}$ and a function $\psi \in C_{\gamma}(H)$ such that $u_{t_{0}}^{(n)} \rightarrow \psi$ in $C([-\bar{T}, 0] ; H)$ for each $\bar{T}>0$. 
Proof. Let $t_{0},\left\{\tau_{n}\right\}_{n \geqslant 1},\left\{\phi_{n}\right\}_{n \geqslant 1}$ and $u^{(n)}=u^{(n)}\left(\cdot ; \tau_{n}, \phi_{n}\right)$ be given as in this lemma. Consider two arbitrary positive numbers $\bar{T}, T$ with $T>\bar{T}$. Let $n_{0}=n_{0}\left(t_{0}, T\right)$ be the positive integer such that $\tau_{n}<t_{0}-T$ for all $n \geqslant n_{0}$. From (3.3) and (3.14)-(3.15) we deduce that for $t \in\left[t_{0}-T, t_{0}\right]$ and $n \geqslant n_{0}$,

$$
\begin{aligned}
\left\|u^{n}(t)\right\|^{2} & \lesssim R\left(t_{0}, T\right):=1+e^{-\sigma\left(t_{0}-T\right)} \int_{-\infty}^{t_{0}} e^{\sigma s}\|f(s)\|^{2} \mathrm{~d} s<+\infty \\
\left\|u_{t}^{n}\right\|_{\gamma}^{2} \lesssim R\left(t_{0}, T\right) . &
\end{aligned}
$$

We next divide two steps to establish that the conditions of the Ascoli-Arzelà type theorem are satisfied.

Step one. We first prove that

$$
\left\{u^{(n)}(t)\right\}_{n \geqslant n_{0}} \text { is precompact in } H \text { for each } t \in\left[t_{0}-\bar{T}, t_{0}\right] .
$$

By Theorem 2.1, we conclude that $u^{(n)}(t) \in D(A)$ for all $t \in\left[t_{0}-T, t_{0}\right]$, whence,

$$
\begin{aligned}
& \frac{1}{2} \frac{\mathrm{d}}{\mathrm{d} t}\left\|u^{(n)}(t)\right\|_{V}^{2}+\nu\left\|A u^{(n)}(t)\right\|^{2}+b_{N}\left(u^{(n)}(t), u^{(n)}(t), A u^{(n)}(t)\right) \\
= & \left(f(t), A u^{(n)}(t)\right)+\left(g\left(t, u^{(n)}(t-\rho(t))\right), A u^{(n)}(t)\right), \quad t \in\left[t_{0}-T, t_{0}\right] .
\end{aligned}
$$

Using Young's inequality, (2.1) and (2.6), we have

$$
\begin{aligned}
& \left(f(t), A u^{(n)}(t)\right) \lesssim \frac{\nu}{8}\left\|A u^{(n)}(t)\right\|^{2}+\|f(t)\|^{2}, \\
& \left(g\left(t, u^{(n)}(t-\rho(t))\right), A u^{(n)}(t)\right) \lesssim \frac{\nu}{8}\left\|A u^{(n)}(t)\right\|^{2}+L_{g}^{2}(t) e^{2 \gamma \rho(t)}\left\|u_{t}^{(n)}\right\|_{\gamma}^{2}, \\
& \left|b_{N}\left(u^{(n)}(t), u^{(n)}(t), A u^{(n)}(t)\right)\right| \lesssim\left\|u^{(n)}(t)\right\|_{V}^{1 / 2}\left\|A u^{(n)}(t)\right\|^{3 / 2} \lesssim \frac{\nu}{4}\left\|A u^{(n)}(t)\right\|^{2}+\left\|u^{(n)}(t)\right\|_{V}^{2} .
\end{aligned}
$$

Combining (3.19)-(3.22) gives

$$
\frac{\mathrm{d}}{\mathrm{d} t}\left\|u^{(n)}\right\|_{V}^{2}+\nu\left\|A u^{(n)}(t)\right\|^{2} \lesssim\|f(t)\|^{2}+2 L_{g}^{2}(t) e^{2 \gamma \rho(t)}\left\|u_{t}^{(n)}\right\|_{\gamma}^{2}+\left\|u^{(n)}(t)\right\|_{V}^{2} .
$$

Integrating (3.23) over $[s, t]$ with $t_{0}-T \leqslant s \leqslant t \leqslant t_{0}$ yields

$$
\left\|u^{(n)}(t)\right\|_{V}^{2} \lesssim\left\|u^{(n)}(s)\right\|_{V}^{2}+\int_{t_{0}-T}^{t_{0}}\left[\left(\|f(\theta)\|^{2}+2 L_{g}^{2}(\theta) e^{2 \gamma \rho(\theta)}\left\|u_{\theta}^{(n)}\right\|_{\gamma}^{2}\right)+\left\|u^{(n)}(\theta)\right\|_{V}^{2}\right] \mathrm{d} \theta .
$$

Now integrating (3.24) with respect to $s$ over $\left[t_{0}-T, t\right]$, we obtain for $t \in\left[t_{0}-\bar{T}, t_{0}\right]$ that

$$
\begin{aligned}
& (T-\bar{T})\left\|u^{(n)}(t)\right\|_{V}^{2} \leqslant\left(t+T-t_{0}\right)\left\|u^{(n)}(t)\right\|_{V}^{2} \\
\lesssim & T \int_{t_{0}-T}^{t_{0}}\left\|u^{(n)}(s)\right\|_{V}^{2} \mathrm{~d} s+T \int_{t_{0}-T}^{t_{0}}\left(\|f(s)\|^{2}+L_{g}^{2}(s) e^{2 \gamma \rho(s)}\left\|u_{s}^{(n)}\right\|_{\gamma}^{2}\right) \mathrm{d} s .
\end{aligned}
$$

In addition, from (3.8) and (3.14), we can get

$$
\begin{aligned}
\int_{\tau}^{t}\left\|u^{(n)}(s)\right\|_{V}^{2} \mathrm{~d} s \lesssim & \left(1+\int_{\tau}^{t} L_{g}(s) e^{\gamma \rho(s)} e^{2 \int_{\tau}^{s} L_{g}(\sigma) e^{\gamma \rho(\sigma)} \mathrm{d} \sigma} \mathrm{d} s\right)\|\phi\|_{\gamma}^{2} \\
& +\int_{\tau}^{t} e^{-2(\nu-\mu) \lambda_{1}(\tau-s)}\|f(s)\|^{2} \mathrm{~d} s \\
& +\int_{\tau}^{t} L_{g}(s) e^{\gamma \rho(s)} \int_{\tau}^{s} e^{-2(\nu-\mu) \lambda_{1}(\tau-\sigma)+2 \int_{\sigma}^{s} L_{g}(\theta) e^{\gamma \rho(\theta)} \mathrm{d} \theta}\|f(\sigma)\|^{2} \mathrm{~d} \sigma \mathrm{d} s .
\end{aligned}
$$


Note that we have $u^{(n)}\left(t ; \tau^{(n)}, \phi_{(n)}\right)=U\left(t, t_{0}-T\right) u\left(t_{0}-T ; \tau_{n}, \phi_{(n)}\right)$. Thus from (3.8)-(3.9), (3.13) and (3.15), we know that

$$
\text { the sequence }\left\{u^{(n)}\right\}_{n \geqslant n_{0}} \text { is bounded in } L^{\infty}\left(t_{0}-\bar{T}, t_{0} ; V\right) \text {. }
$$

Therefore, it follows from the compact embedding $V \hookrightarrow H$ and the continuity of $u^{(n)}$ (see (2.10)) that the set

$$
\left\{u^{n}(t): t \in\left[t_{0}-\bar{T}, t_{0}\right], n \geqslant n_{0}\right\} \text { is precompact in } H,
$$

from which (3.18) immediately follows.

Step two. We establish the equicontinuity of $\left\{u^{(n)}\right\}_{n \geqslant n_{0}}$ on $\left[t_{0}-\bar{T}, t_{0}\right]$ by contradiction.

Suppose that this equicontinuity does not hold true, and then there exists a positive constant $\varepsilon_{0}$ and two sequences $\left\{t_{n}^{(1)}\right\}$ and $\left\{t_{n}^{(2)}\right\}$, satisfying $t_{0}-\bar{T} \leqslant t_{n}^{(1)} \leqslant t_{n}^{(2)} \leqslant t_{0}$ and $\left|t_{n}^{(2)}-t_{n}^{(1)}\right| \leqslant \frac{1}{n}$, such that

$$
\left\|u^{(n)}\left(t_{n}^{(1)}\right)-u^{(n)}\left(t_{n}^{(2)}\right)\right\| \geqslant \varepsilon_{0} .
$$

By (3.28), we can assume that $t_{n}^{(1)} \rightarrow t^{*}, u^{(n)}\left(t^{*}\right) \rightarrow z^{*}$ and $u^{(n)}\left(t_{n}^{(i)}\right) \rightarrow z^{(i)}(i=1,2)$ in $H$ as $n \rightarrow+\infty$. Evidently, $t_{n}^{(2)} \rightarrow t^{*}$ as $n \rightarrow+\infty$ and $t^{*} \in\left[t_{0}-\bar{T}, t_{0}\right]$. By (3.23), we know that

$$
\left\|z^{(1)}-z^{(2)}\right\| \geqslant \varepsilon_{0} .
$$

Set $y^{(n)}(t)=u^{(n)}(t)-u^{(n)}\left(t^{*}\right)$ for $n \geqslant n_{0}$. Then we obtain

$$
\begin{aligned}
& \frac{1}{2} \frac{\mathrm{d}}{\mathrm{d} t}\left\|y^{(n)}(t)\right\|^{2}+\nu\left\|y^{(n)}(t)\right\|_{V}^{2}+\nu \int_{\Omega} \nabla u^{(n)}\left(t^{*}\right) \cdot \nabla y^{(n)}(t) \mathrm{d} x+b_{N}\left(u^{(n)}(t), u^{(n)}(t), y^{(n)}(t)\right) \\
= & \left(f(t), y^{(n)}(t)\right)+\left(g\left(t, u^{(n)}(t-\rho(t))\right), y^{(n)}(t)\right), \quad t \in\left[t_{0}-\bar{T}, t_{0}\right] .
\end{aligned}
$$

Applying Schwartz's inequality and (g3), we have

$$
\begin{aligned}
\left|\int_{\Omega} \nabla u^{(n)}\left(t^{*}\right) \cdot \nabla y^{(n)}(t) \mathrm{d} x\right| & \leqslant \frac{1}{2}\left\|u^{(n)}\left(t^{*}\right)\right\|_{V}^{2}+\frac{1}{2}\left\|y^{(n)}(t)\right\|_{V}^{2}, \\
2\left|\left(f(t), y^{(n)}(t)\right)\right| & \leqslant\|f(t)\|^{2}+\left\|y^{(n)}(t)\right\|^{2}, \\
2\left|\left(g\left(t, u^{(n)}(t-\rho(t))\right), y^{(n)}(t)\right)\right| & \leqslant L_{g}^{2}(t) e^{2 \gamma \rho(t)}\left\|u_{t}^{(n)}\right\|_{\gamma}^{2}+\left\|y^{(n)}(t)\right\|^{2} .
\end{aligned}
$$

At the same time, by (2.3)-(2.6) and (2.7), we see that

$$
\begin{aligned}
\left.\mid b_{N}\left(u^{(n)}(t), u^{(n)}(t)\right), y^{(n)}(t)\right) \mid & =\left|b_{N}\left(y^{(n)}(t), u^{(n)}(t), y^{(n)}(t)\right)-b_{N}\left(u^{(n)}\left(t^{*}\right), u^{(n)}(t), u^{(n)}\left(t^{*}\right)\right)\right| \\
& \lesssim\left\|y^{(n)}(t)\right\|_{V}^{3 / 2}\left\|y^{(n)}(t)\right\|^{1 / 2}+\left\|u^{(n)}\left(t^{*}\right)\right\|_{V}^{2} \\
& \lesssim \frac{\nu}{2}\left\|y^{(n)}(t)\right\|_{V}^{2}+\left\|y^{(n)}(t)\right\|^{2}+\left\|u^{(n)}\left(t^{*}\right)\right\|_{V}^{2} .
\end{aligned}
$$

Taking (3.31)-(3.35) into account yields

$$
\frac{\mathrm{d}}{\mathrm{d} t}\left\|y^{(n)}(t)\right\|^{2} \lesssim\left\|y^{(n)}(t)\right\|^{2}+\|f(t)\|^{2}+L_{g}^{2}(t) e^{2 \gamma \rho(t)}\left\|u_{t}^{(n)}\right\|_{\gamma}^{2}+\left\|u^{(n)}\left(t^{*}\right)\right\|_{V}^{2} .
$$


Integrating (3.36) from $t^{*}$ to $t_{n}^{(i)}$ and using (3.16)- (3.17) gives

$$
\begin{aligned}
\left\|y^{(n)}\left(t_{n}^{i}\right)\right\|^{2} \leqslant & {\left[R\left(t_{0}, T\right)+c_{1}\right]\left|t_{n}^{i}-t^{*}\right|+\left|\int_{t^{*}}^{t_{n}^{i}}\|f(s)\|^{2} \mathrm{~d} s\right| } \\
& +R\left(t_{0}, T\right)\left|\int_{t^{*}}^{t_{n}^{i}} L_{g}^{2}(s) e^{2 \gamma \rho(s)} \mathrm{d} s\right|
\end{aligned}
$$

where (see (2.10) and (3.27))

$$
c_{1}:=\sup _{t \in\left[t_{0}-\bar{T}, t_{0}\right]}\left\{\left\|u_{n}(t)\right\|_{V}: n \geqslant n_{0}\right\}
$$

Letting $n \rightarrow+\infty$ in (3.37) and using the assumptions $f \in L_{\mathrm{loc}}^{2}\left(\mathbb{R} ; \mathbb{L}^{2}(\Omega)\right)$ and (g3), we conclude that

$$
\left\|z^{i}-z^{*}\right\|^{2}=\lim _{n \rightarrow+\infty}\left\|u^{(n)}\left(t_{n}^{i}\right)-u^{(n)}\left(t^{*}\right)\right\|^{2}=0, \quad \text { for } i=1,2,
$$

which contradicts (3.30).

At this stage, we can apply the Ascoli-Arzelà type theorem [1] to claim that for each $\bar{T}>0$, $\left\{u^{(n)}\right\}_{n \in \mathbb{N}_{+}}$is precompact in $C\left(\left[t_{0}-\bar{T}, t_{0}\right] ; H\right)$. Thus there exists a function $\psi \in C([-\bar{T}, 0] ; H)$ and a subsequence of $u_{t_{0}}^{(n)}$ such that $\left.u_{t_{0}}^{(n)}\right|_{[-\bar{T}, 0]} \rightarrow \psi$ in $C([-\bar{T}, 0] ; H)$. Repeating this procedure for $n \bar{T}$ with $n=2,3, \cdots$, and using the diagonal procedure, we can obtain a function $\psi \in C((-\infty, 0] ; H)$ such that (extract a subsequence if necessary) $\left.u_{t_{0}}^{(n)}\right|_{[-\bar{T}, 0]} \rightarrow \psi$ in $C([-\bar{T}, 0] ; H)$ for each positive number $\bar{T}$. The proof of Lemma 3.2 is complete.

Lemma 3.3. Let the assumptions (H1) and (H2) hold. Then the process $\{U(t, \tau)\}_{t \geqslant \tau}$ is pullback $\mathcal{D}_{\sigma^{-}}$ asymptotically compact in $C_{\gamma}(H)$.

Proof. We continue to use the notations of the previous lemma. To establish the pullback $\mathcal{D}_{\sigma}$-asymptotic compactness, we shall prove that $u_{t_{0}}^{n} \rightarrow \psi$ in $C_{\gamma}(H)$, for which, it is sufficient to show that, for every $\varepsilon>0$, there exists some positive integer $n_{\varepsilon}$ such that

$$
\sup _{s \in(-\infty, 0]} e^{2 \gamma s}\left\|u_{t_{0}}^{(n)}(s)-\psi(s)\right\|^{2}<\varepsilon, \quad \text { for all } n \geqslant n_{\varepsilon} .
$$

Indeed, by (3.16)-(3.17) and the convergence proved in the previous lemma, we have that

$$
\|\psi(s)\|^{2} \lesssim 1+e^{-\sigma\left(t_{0}-T\right)} \int_{-\infty}^{t_{0}} e^{\sigma s}\|f(s)\|^{2} \mathrm{~d} s=R\left(t_{0}, T\right), \quad \text { for all } s \in[-T, 0] \text { and } T>0 .
$$

The rest proof is similar to that of [31, Proposition 3] and we omit the details here.

At this stage we combine Lemmas 3.1, 3.3 and the general result of [19, Theorem 3.11] to obtain the main result of this section, namely,

Theorem 3.1. Let the assumptions $(\mathbf{H 1})$ and $(\mathbf{H 2})$ hold. Then the process $\{U(t, \tau)\}_{t \geqslant \tau}$ possesses a pullback $\mathcal{D}_{\sigma}$-attractor (denoted by) $\hat{\mathcal{A}}_{\mathcal{D}_{\sigma}}^{H}=\left\{\mathcal{A}_{\mathcal{D}_{\sigma}}^{H}(t): t \in \mathbb{R}\right\}$ in $C_{\gamma}(H)$. 


\section{Existence of the invariant Borel probability measures in $C_{\gamma}(H)$}

In this section, we first aim to prove that for each given $t \in \mathbb{R}$ and $\phi \in C_{\gamma}(H)$, the $C_{\gamma}(H)$-valued function $\tau \mapsto U(t, \tau) \phi$ is bounded and continuous on $(-\infty, t]$. Then we combine Theorem 3.1 and the abstract result of [28, Theorem 3.1] to obtain the existence of invariant Borel probability measures for $\{U(t, \tau)\}_{t \geqslant \tau}$ in $C_{\gamma}(H)$.

Lemma 4.1. Let the assumptions $(\mathbf{H 1})$ and (H2) hold. Then for every $\phi \in C_{\gamma}(H)$ and every $t \in \mathbb{R}$ the $C_{\gamma}(H)$-valued function $\tau \mapsto U(t, \tau) \phi$ is bounded on $(-\infty, t]$.

Proof. Let $\phi \in C_{\gamma}(H)$ and $t \in \mathbb{R}$ be given. Note (3.4) is equivalent to (see [31, Remark 5])

$$
\int_{-\infty}^{t} e^{\sigma s}\|f(s)\|^{2} \mathrm{~d} s<+\infty \quad \text { for all } t \in \mathbb{R} \text {. }
$$

Then (3.14) and (4.1) imply that for all $\tau \in(-\infty, t]$,

$$
\|U(t, \tau) \phi\|_{\gamma}^{2} \lesssim e^{-\sigma(t-\tau)}\|\phi\|_{\gamma}^{2}+\int_{\tau}^{t} e^{-\sigma(t-s)}\|f(s)\|^{2} \mathrm{~d} s \leqslant\|\phi\|_{\gamma}^{2}+e^{-\sigma t} \int_{-\infty}^{t} e^{\sigma s}\|f(s)\|^{2} \mathrm{~d} s,
$$

the right-hand side of which is bounded by a positive constant independent of $\tau \in(-\infty, t]$.

In the sequel, we set a positive number $M(t, \phi)$ such that

$$
M^{2}(t, \phi):=\|\phi\|_{\gamma}^{2}+e^{-\sigma t} \int_{-\infty}^{t} e^{\sigma s}\|f(s)\|^{2} \mathrm{~d} s .
$$

Then $M^{2}(t, \phi)$ is a continuous function with respect to $t \in \mathbb{R}$.

To establish the continuity of the $C_{\gamma}(H)$-valued function $\tau \mapsto U(t, \tau) \phi$ on $(-\infty, t]$, we next prove two auxiliary lemmas.

Lemma 4.2. Let $\phi \in C_{\gamma}(H)$ be given. Then for each $\varepsilon>0$, there exists some $\delta_{1}=\delta_{1}(\varepsilon, \phi)>0$ such that for all $s_{1}, s_{2} \in(-\infty, 0]$ with $\left|s_{1}-s_{2}\right|<\delta_{1}$ there holds

$$
e^{\gamma s_{2}}\left\|\phi\left(s_{1}\right)-\phi\left(s_{2}\right)\right\|<\varepsilon
$$

Proof. By the definition of $C_{\gamma}(H)$, we set $\phi_{\infty}:=\lim _{s \rightarrow-\infty} e^{\gamma s} \phi(s) \in H$. Then there exists some $s_{0}<0$ such that

$$
\left\|e^{\gamma s} \phi(s)-\phi_{\infty}\right\|<\frac{\varepsilon}{4}, \forall s \leqslant s_{0}
$$

and thus

$$
\left\|e^{\gamma s_{1}} \phi\left(s_{1}\right)-e^{\gamma s_{2}} \phi\left(s_{2}\right)\right\| \leqslant\left\|e^{\gamma s_{1}} \phi\left(s_{1}\right)-\phi_{\infty}\right\|+\left\|e^{\gamma s_{2}} \phi\left(s_{2}\right)-\phi_{\infty}\right\|<\frac{\varepsilon}{2}, \forall s_{1}, s_{2} \leqslant s_{0} .
$$

On the other hand, the $H$-valued function $s \mapsto e^{\gamma s} \phi(s)$ is uniformly continuous on the interval $\left[s_{0}-1,0\right]$, whence there is some $\delta_{1}^{\prime} \in(0,1)$ such that

$$
\left\|e^{\gamma s_{1}} \phi\left(s_{1}\right)-e^{\gamma s_{2}} \phi\left(s_{2}\right)\right\|<\frac{\varepsilon}{2} \quad \text { for } s_{1}, s_{2} \in\left[s_{0}-1,0\right] \text { with }\left|s_{1}-s_{2}\right|<\delta_{1}^{\prime} .
$$


Letting $\delta_{1}=\min \left\{\delta_{1}^{\prime}, \frac{1}{\gamma} \ln \left(1+\frac{\varepsilon}{2\|\phi\|_{\gamma}}\right)\right\}$, we have by (4.3) and (4.4) that for all $s_{1}, s_{2} \in(-\infty, 0]$ with $\left|s_{1}-s_{2}\right|<\delta_{1}$ there holds

$$
\begin{aligned}
& e^{\gamma s_{2}}\left\|\phi\left(s_{1}\right)-\phi\left(s_{2}\right)\right\| \leqslant\left\|e^{\gamma s_{1}} \phi\left(s_{1}\right)-e^{\gamma s_{2}} \phi\left(s_{2}\right)\right\|+\left|e^{\gamma s_{1}}-e^{\gamma s_{2}}\right|\left\|\phi\left(s_{1}\right)\right\| \\
\leqslant & \frac{\varepsilon}{2}+\left|e^{\gamma\left(s_{2}-s_{1}\right)}-1\right|\|\phi\|_{\gamma}<\varepsilon .
\end{aligned}
$$

The proof is complete.

Lemma 4.3. Let the assumptions (H1) and (H2) hold, and $\tau_{*} \in \mathbb{R}$ and $\phi \in C_{\gamma}(H)$ be given. Then for each $\varepsilon>0$, there exists some $\delta_{2}=\delta_{2}\left(\varepsilon, \tau_{*}, \phi\right)>0$ such that

$$
\|u(s ; \tau, \phi)-\phi(0)\|<\varepsilon, \quad \text { for all } \tau \in\left(\tau_{*}-\delta_{2}, \tau_{*}\right) \text { and } s \in\left[\tau, \tau_{*}\right],
$$

where $u(s ; \tau, \phi)$ is the solution of the problem (1.1) with the initial datum $\phi$ at the initial time $\tau$.

Proof. Let $\tau_{*} \in \mathbb{R}$ and $\phi \in C_{\gamma}(H)$ be given. Firstly, we prove that

$$
\int_{\tau}^{\tau_{*}}\left\|\frac{\mathrm{d} u(\theta ; \tau, \phi)}{\mathrm{d} \theta}\right\|_{V^{\prime}}^{2} \mathrm{~d} \theta \leqslant c_{2}
$$

where

$$
c_{2}:=\|\phi\|_{\gamma}^{2}+\int_{\tau_{*}-1}^{\tau_{*}}\|f(\theta)\|^{2} \mathrm{~d} \theta+\sup _{\left[\tau_{*}-1, \tau_{*}\right]} M^{2}(\theta, \phi),
$$

is a bounded quantity depending on $\tau_{*}, g, f, \cdots$, etc., but being independent of $\tau \in\left[\tau_{*}-1, \tau_{*}\right]$. Indeed, from (2.9) we can see that

$$
\begin{gathered}
\left\|\frac{\mathrm{d} u(\theta ; \tau, \phi)}{\mathrm{d} \theta}\right\|_{V^{\prime}}^{2} \lesssim \\
+\|A u(\theta ; \tau, \phi)\|_{V^{\prime}}^{2}+\left\|B_{N}(u(\theta ; \tau, \phi), u(\theta ; \tau, \phi))\right\|_{V^{\prime}}^{2} \\
+\|f(\theta)\|^{2}+\|g(\theta, u(\theta-\rho(\theta)))\|^{2} .
\end{gathered}
$$

By (2.5), (g2), (g3) and some simple observations, we have

$$
\left\{\begin{array}{l}
\|A u(\theta ; \tau, \phi)\|_{V^{\prime}}^{2} \lesssim\|u(\theta ; \tau, \phi)\|_{V}^{2}, \\
\left\|B_{N}(u(\theta ; \tau, \phi), u(\theta ; \tau, \phi))\right\|_{V^{\prime}}^{2} \lesssim\|u(\theta ; \tau, \phi)\|_{V}^{2} \\
\|g(\theta, u(\theta-\rho(\theta)))\|^{2} \lesssim \sup _{\left[\tau_{*}-1, \tau_{*}\right]} M^{2}(\theta, \phi) .
\end{array}\right.
$$

Inserting (4.7) and (4.8) into (4.6), then using (3.3), (3.5) and (3.26) gives

$$
\begin{aligned}
\int_{\tau}^{\tau_{*}}\left\|\frac{\mathrm{d} u(\theta ; \tau, \phi)}{\mathrm{d} \theta}\right\|_{V^{\prime}}^{2} \mathrm{~d} \theta & \left.\lesssim \int_{\tau}^{\tau_{*}} \| u(\theta ; \tau, \phi)\right)\left\|_{V}^{2} \mathrm{~d} \theta+\int_{\tau}^{\tau_{*}}\right\| f(\theta) \|^{2} \mathrm{~d} \theta+\left(\tau_{*}-\tau\right) \sup _{\left[\tau_{*}-1, \tau_{*}\right]} M^{2}(\theta, \phi) \\
& \lesssim\|\phi\|_{\gamma}^{2}+\int_{\tau_{*}-1}^{\tau_{*}}\|f(\theta)\|^{2} \mathrm{~d} \theta+\sup _{\left[\tau_{*}-1, \tau_{*}\right]} M^{2}(\theta, \phi), \forall \tau \in\left[\tau_{*}-1, \tau_{*}\right]
\end{aligned}
$$

that is (4.6) is proved. 
Secondly, we observe that for $\tau_{*}-1 \leqslant \tau<s \leqslant \tau_{*}$ there holds

$$
\begin{aligned}
& \|u(s ; \tau, \phi)-\phi(0)\|^{2} \\
= & \|u(s ; \tau, \phi)\|^{2}-\|\phi(0)\|^{2}-2(u(s ; \tau, \phi)-\phi(0), \phi(0)) \\
= & \int_{\tau}^{s} \frac{\mathrm{d}\|u(\theta ; \tau, \phi)\|^{2}}{\mathrm{~d} \theta} \mathrm{d} \theta-2(u(s ; \tau, \phi)-\phi(0), \phi(0)) .
\end{aligned}
$$

By (3.3) and (3.13), we obtain

$$
\begin{aligned}
\left|\int_{\tau}^{s} \frac{\mathrm{d}\|u(\theta ; \tau, \phi)\|^{2}}{\mathrm{~d} \theta} \mathrm{d} \theta\right| & \lesssim \int_{\tau}^{s}\|f(\theta)\|^{2} \mathrm{~d} \theta+\int_{\tau}^{s} L_{g}(\theta) e^{\gamma \rho(\theta)}\left\|u_{\theta}\right\|_{\gamma}^{2} \mathrm{~d} \theta \\
& \lesssim \int_{\tau}^{\tau_{*}}\|f(\theta)\|^{2} \mathrm{~d} \theta+\left(\tau_{*}-\tau\right) \sup _{\theta \in\left[\tau_{*}-1, \tau_{*}\right]} M^{2}(\theta, \phi) .
\end{aligned}
$$

Notice that $f \in L_{\mathrm{loc}}^{2}\left(\mathbb{R}, \mathbb{L}^{2}(\Omega)\right)$, and $\sup _{\theta \in\left[\tau_{*}-1, \tau_{*}\right]} M^{2}(\theta, \phi)$ is a constant independent of $\tau$. Therefore, from (4.11) we see that there exists some $\delta_{2}^{\prime}=\delta_{2}^{\prime}\left(\varepsilon, \tau_{*}, \phi\right) \in(0,1)$ such that

$$
\left|\int_{\tau}^{s} \frac{\mathrm{d}\|u(\theta ; \tau, \phi)\|^{2}}{\mathrm{~d} \theta} \mathrm{d} \theta\right|<\frac{\varepsilon^{2}}{2}, \quad \tau_{*}-\delta_{2}^{\prime}<\tau<s \leqslant \tau_{*} .
$$

At the same time, since $\phi(0) \in H$ and $V$ is dense in $H$, there exists some $\psi \in V$ such that

$$
\|\psi-\phi(0)\|<\varepsilon^{2} / 16 \sup _{\theta \in\left[\tau_{*}-1, \tau_{*}\right]} M^{2}(\theta, \phi) .
$$

Using (4.6),

$$
\begin{aligned}
|(u(s ; \tau, \phi)-\phi(0), \phi(0))| & \leqslant|(u(s ; \tau, \phi)-\phi(0), \phi(0)-\psi)|+|(u(s ; \tau, \phi)-\phi(0), \psi)| \\
& \leqslant 2 \sup _{\theta \in\left[\tau_{*}-1, \tau_{*}\right]} M(\tau, \phi)\|\phi(0)-\psi\|+|\langle u(s ; \tau, \phi)-\phi(0), \psi\rangle| \\
& <\frac{\varepsilon^{2}}{8}+\left|\left\langle\int_{\tau}^{s} \frac{\mathrm{d} u(\theta ; \tau, \phi)}{\mathrm{d} \theta} \mathrm{d} \theta, \psi\right\rangle\right| \\
& \leqslant \frac{\varepsilon^{2}}{8}+\|\psi\|_{V} \sqrt{\tau_{*}-\tau}\left(\int_{\tau}^{\tau_{*}}\left\|\frac{\mathrm{d} u(\theta ; \tau, \phi)}{\mathrm{d} \theta}\right\|_{V^{\prime}}^{2} \mathrm{~d} \theta\right)^{\frac{1}{2}} \\
& \leqslant \frac{\varepsilon^{2}}{8}+\sqrt{c_{2}}\|\psi\|_{V} \sqrt{\tau_{*}-\tau},
\end{aligned}
$$

which implies that there exists some $\delta_{2}^{\prime \prime} \in(0,1)$, independent of $\tau$, such that

$$
|(u(s ; \tau, \phi)-\phi(0), \phi(0))| \leqslant \frac{\varepsilon^{2}}{4}, \quad \tau_{*}-\delta_{2}^{\prime \prime}<\tau<s \leqslant \tau_{*} .
$$

Picking $\delta_{2}=\min \left\{\delta_{2}^{\prime}, \delta_{2}^{\prime \prime}\right\}$, the desired result follows from (4.10), (4.12) and (4.14). The proof of Lemma 4.3 is complete.

Lemma 4.4. Let the assumptions (H1) and (H2) hold. Then for every $\phi \in C_{\gamma}(H)$ and every $t \in \mathbb{R}$ the $C_{\gamma}(H)$-valued function $\tau \mapsto U(t, \tau) \phi$ is continuous on $(-\infty, t]$. 
Proof. Let $\phi \in C_{\gamma}(H)$ and $t \in \mathbb{R}$ be given. We shall prove that for each given $\tau_{*} \in(-\infty, t]$ the $C_{\gamma}(H)$-valued function $\tau \mapsto U(t, \tau) \phi$ is both left and right continuous at $\tau=\tau_{*}$. We next prove the left continuity at $\tau=\tau_{*}$.

Firstly, on one hand, for every $\varepsilon>0$ we deduce from Lemma 4.2 that there exists some $\delta_{3}^{\prime}=$ $\delta_{3}^{\prime}(\varepsilon, \phi)>0$ such that, for all $s_{1}, s_{2} \in(-\infty, 0]$ with $\left|s_{1}-s_{2}\right|<\delta_{1}$, there holds

$$
e^{\gamma s_{2}}\left\|\phi\left(s_{1}\right)-\phi\left(s_{2}\right)\right\|<\varepsilon / 2 .
$$

On the other hand, by Lemma 4.3 , there is a $\delta_{3}^{\prime \prime}=\delta_{3}^{\prime \prime}\left(\varepsilon, \tau_{*}, \phi\right)>0$ such that whenever $\tau \in\left(\tau_{*}-\delta_{3}^{\prime \prime}, \tau_{*}\right)$ there holds

$$
\|u(s ; \tau, \phi)-\phi(0)\|<\varepsilon / 2, \quad \text { for all } s \in\left[\tau, \tau_{*}\right] .
$$

Thus, for the positive $\varepsilon$ above, there exists some $\delta_{3}=\delta_{3}\left(\varepsilon, \tau_{*}, \phi\right)=\min \left\{\delta_{3}^{\prime}, \delta_{3}^{\prime \prime}\right\}>0$ such that

$$
\begin{aligned}
\left\|u_{*}(\theta)-u(\theta)\right\| & =\|\phi(\theta-\tau)-u(\theta)\| \\
& \leqslant\|\phi(\theta-\tau)-\phi(0)\|+\|\phi(0)-u(\theta ; \tau, \phi)\|<\varepsilon, \quad \tau_{*}-\delta_{3}<\tau<\tau_{*}, \quad \theta \in\left[\tau, \tau_{*}\right],
\end{aligned}
$$

which implies that

$$
\max _{\theta \in\left[\tau, \tau_{*}\right]}\left\|u_{*}\left(\theta ; \tau_{*}, \phi\right)-u(\theta ; \tau, \phi)\right\|<\varepsilon, \quad \tau_{*}-\delta_{3}<\tau<\tau_{*}, \quad \theta \in\left[\tau, \tau_{*}\right],
$$

where $u_{*}\left(\theta ; \tau_{*}, \phi\right)$ is the solution corresponding to the initial datum $\phi$ at the initial time $\tau_{*}$.

Secondly, by (2.18) and (3.3) we have

$$
\begin{aligned}
\max _{\theta \in\left[\tau_{*}, t\right]}\left\|u_{*}\left(\theta ; \tau_{*}, \phi\right)-u(\theta ; \tau, \phi)\right\| \\
\quad \leq\left\|\phi(0)-u\left(\tau_{*} ; \tau, \phi\right)\right\|^{2} \exp \left(\int_{\tau_{*}}^{t} L_{g}(s) e^{\gamma \rho(s)} \mathrm{d} s+\left(t-\tau_{*}\right)\right) \\
\quad+\left\|\phi-u_{\tau_{*}}\right\|_{\gamma}^{2} \int_{\tau_{*}}^{t} L_{g}(s) e^{\gamma \rho(s)} \exp \left(\int_{\tau_{*}}^{s} L_{g}(\theta) e^{\gamma \rho(\theta)} \mathrm{d} \theta+\left(s-\tau_{*}\right)\right) \mathrm{d} s . \\
\quad \lesssim\left\|\phi(0)-u\left(\tau_{*} ; \tau, \phi\right)\right\|^{2}+\left\|\phi-u_{\tau_{*}}\right\|_{\gamma}^{2}
\end{aligned}
$$

using the representation $u(\theta ; \tau, \phi)=u\left(\theta ; \tau_{*}, u\left(\tau_{*} ; \tau, \phi\right)\right)$ and the fact that $t$ and $\tau_{*}$ are fixed. Now by Lemma 4.3, we derive that for above $\varepsilon>0$ there exists $\delta_{4}^{\prime}=\delta_{4}^{\prime}\left(\varepsilon, t, \tau_{*}, \phi\right)>0$ such that

$$
\left\|\phi(0)-u\left(\tau_{*} ; \tau, \phi\right)\right\|<\varepsilon / 2, \text { for } \tau_{*}-\delta_{4}^{\prime}<\tau<\tau_{*} .
$$

At the same time, by (4.15) and (4.17), there exists $\delta_{4}^{\prime \prime}=\delta_{4}^{\prime \prime}\left(\varepsilon, \tau_{*}, \phi\right)>0$ such that when $\tau_{*}-\delta_{4}^{\prime \prime}<\tau<$ $\tau_{*}$ then

$$
\begin{aligned}
\left\|\phi-u_{\tau_{*}}\right\|_{\gamma} & \leqslant \max \left\{\sup _{s \leqslant \tau_{*}-\tau} e^{\gamma s}\left\|\phi(s)-u_{\tau_{*}}(s)\right\|, \sup _{s \in\left[\tau-\tau_{*}, 0\right]} e^{\gamma s}\left\|\phi(s)-u_{\tau_{*}}(s)\right\|\right\} \\
& \leqslant \max \left\{\sup _{s \leqslant \tau-\tau_{*}} e^{\gamma s}\left\|\phi(s)-\phi\left(s+\tau_{*}-\tau\right)\right\|, \sup _{s \in\left[\tau, \tau_{*}\right]}\left\|u_{*}(s)-u(s)\right\|\right\} \\
& \leqslant \max \left\{\sup _{r \leqslant 0} e^{\gamma\left(r+\tau-\tau_{*}\right)}\left\|\phi\left(r+\tau-\tau_{*}\right)-\phi(r)\right\|, \varepsilon\right\}<\varepsilon / 2 .
\end{aligned}
$$


Picking $\delta_{4}=\delta_{4}\left(\varepsilon, t, \tau_{*}, \phi\right)=\min \left\{\delta_{4}^{\prime}, \delta_{4}^{\prime \prime}\right\}$, then (4.18)-(4.20) imply

$$
\max _{\theta \in\left[\tau_{*}, t\right]}\left\|u_{*}\left(\theta ; \tau_{*}, \phi\right)-u(\theta ; \tau, \phi)\right\|<\varepsilon, \quad \tau_{*}-\delta_{4}<\tau<\tau_{*}<\varepsilon .
$$

We now choose $\delta=\delta\left(\varepsilon, t, \tau_{*}, \phi\right)=\min \left\{\delta_{3}, \delta_{4}\right\}$, and then deduce from (4.15), (4.17) and (4.21) that if $\tau_{*}-\delta<\tau<\tau_{*}$ then

$$
\begin{aligned}
& \left\|U(t, \tau) \phi-U\left(t, \tau_{*}\right) \phi\right\|_{\gamma}=\sup _{\theta \leqslant 0} e^{\gamma \theta}\left\|u(t+\theta)-u_{*}(t+\theta)\right\|_{\gamma} \\
\leqslant & \max \left\{\sup _{\theta \leqslant \tau-t} e^{\gamma \theta}\left\|\phi\left(\theta+t-\tau_{*}\right)-\phi(\theta+t-\tau)\right\|, \sup _{\theta \in[\tau-t, 0]} e^{\gamma \theta}\left\|u(t+\theta)-u_{*}(t+\theta)\right\|\right\} \\
\leqslant & \max \left\{\sup _{r \leqslant 0} e^{\gamma\left(r+\tau_{*}-t\right)}\left\|\phi(r)-\phi\left(r+\tau_{*}-\tau\right)\right\|, \sup _{\theta \in[\tau, t]}\left\|u(\theta)-u_{*}(\theta)\right\|\right\}<\varepsilon .
\end{aligned}
$$

The left continuity of the $C_{\gamma}(H)$-valued function $\tau \mapsto U(t, \tau) \phi$ at $\tau=\tau_{*}$ is proved. Since the proof of the right continuity of $U(t, \tau) \phi$ at $\tau=\tau_{*}$ is similar to that of the left continuity, we omit the details and end the proof of Lemma 4.4.

To construct the invariant measures for the process $\{U(t, \tau)\}_{t \geqslant \tau}$, we next recall the definition of generalized Banach limits.

Definition 4.1. ( $[16,28])$ A generalized Banach limit is any linear functional, denoted by $\mathrm{LIM}_{t \rightarrow+\infty}$, defined on the space of all bounded real-valued functions on $[0,+\infty)$ and satisfying

(1) $\operatorname{LIM}_{t \rightarrow+\infty} \zeta(t) \geqslant 0$ for nonnegative functions $\zeta(\cdot)$ on $[0,+\infty)$;

(2) $\operatorname{LIM}_{t \rightarrow+\infty} \zeta(t)=\lim _{t \rightarrow+\infty} \zeta(t)$ if the usual limit $\lim _{t \rightarrow+\infty} \zeta(t)$ exists.

Remark 4.1. Notice that we consider the "pullback" asymptotic behavior and we require generalized limits as $\tau \rightarrow-\infty$. For a given real-valued function $\zeta$ defined on $(-\infty, 0]$ and a given Banach limit $\operatorname{LIM}_{t \rightarrow+\infty}$, we define $\operatorname{LIM}_{t \rightarrow-\infty} \zeta(t)=\operatorname{LIM}_{t \rightarrow+\infty} \zeta(-t)$.

The main result of this article reads as follows.

Theorem 4.1. Let the assumptions (H1) and (H2) hold. Let $\{U(t, \tau)\}_{t \geqslant \tau}$ be the process associated to the problem (1.1) and $\widehat{\mathcal{A}}_{\mathcal{D}_{\sigma}}=\left\{\mathcal{A}_{\mathcal{D}_{\sigma}}(t): t \in \mathbb{R}\right\}$ the pullback $\mathcal{D}_{\sigma}$-attractor obtained in Theorem 3.1. Then for a given generalized Banach limit $\operatorname{LIM}_{t \rightarrow+\infty}$ and a continuous map $\xi_{\tau}: \mathbb{R} \longmapsto C_{\gamma}(H)$ with $\xi_{\tau}(\cdot) \in \mathcal{D}_{\sigma}$, there exists a unique family of Borel probability measures $\left\{m_{t}\right\}_{t \in \mathbb{R}}$ in $C_{\gamma}(H)$ such that the support of the measure $m_{t}$ is contained in $\mathcal{A}_{\mathcal{D}_{\sigma}}(t)$ and

$$
\begin{aligned}
\operatorname{LIM}_{\tau \rightarrow-\infty} \frac{1}{t-\tau} \int_{\tau}^{t} \Upsilon\left(U(t, s) \xi_{s}\right) \mathrm{d} s & =\int_{\mathcal{A}_{\mathcal{D}_{\sigma}}(t)} \Upsilon(u) \mathrm{d} m_{t}(u) \\
& =\int_{C_{\gamma}(H)} \Upsilon(u) \mathrm{d} m_{t}(u) \\
& =\operatorname{LIM}_{\tau \rightarrow-\infty} \frac{1}{t-\tau} \int_{\tau}^{t} \int_{C_{\gamma}(H)} \Upsilon(U(t, s) u) \mathrm{d} m_{s}(u) \mathrm{d} s
\end{aligned}
$$


for every real-valued continuous functional $\Upsilon$ on $C_{\gamma}(H)$. Moreover, $m_{t}$ is invariant in the sense that

$$
\int_{\mathcal{A}_{\mathcal{D}_{\sigma}}(t)} \Upsilon(u) \mathrm{d} m_{t}(u)=\int_{\mathcal{A}_{\mathcal{D}_{\sigma}}(\tau)} \Upsilon(U(t, \tau) u) \mathrm{d} m_{\tau}(u), \quad t \geqslant \tau .
$$

Proof. From Theorems 2.1 and 2.2, the solution operators of the problem (1.1) generate a continuous process $\{U(t, \tau)\}_{t \geqslant \tau}$ on the space $C_{\gamma}(H)$. Theorem 3.1 shows that $\{U(t, \tau)\}_{t \geqslant \tau}$ possesses a pullback attractor in $C_{\gamma}(H)$. Lemmas 4.1 and 4.4 indicate that for each given $t \in \mathbb{R}$ and $\phi \in C_{\gamma}(H)$ the $C_{\gamma}(H)$ valued function $\tau \mapsto U(t, \tau) \phi$ is bounded and continuous on $(-\infty, t]$. Using these facts and the abstract result of [28, Theorem 3.1], we obtain the results of Theorem 4.1.

\section{Conclusions and remarks}

In this article, we first prove the global well-posedness of the 3D GMNS equations with unbounded variable delays. Then we establish that its solution operators generate a continuous process $\{U(t, \tau)\}_{t \geqslant \tau}$ on the space $C_{\gamma}(H)$. Furthermore, the process $\{U(t, \tau)\}_{t \geqslant \tau}$ possesses a pullback attractor in $C_{\gamma}(H)$. Finally, we prove the existence of invariant Borel probability measures which are supported by the pullback attractor. There are two points that we would like to point out.

Firstly, we apply the generalized Ascoli-Arzelà theorem to prove the "weak version" of the pullback asymptotic compactness of the process $\{U(t, \tau)\}_{t \geqslant \tau}$ (see Lemma 3.2). This is prompted by the delay phenomenon. Since the problem discussed contains unbounded variable delays, it seems suitable for us to choose $C_{\gamma}(H)$ as the phase space. The Ascoli-Arzelà theorem has been proved to be a powerful tool to investigate the compactness of the sets such as the subset of $C_{\gamma}(H)$.

Secondly, for each given $t \in \mathbb{R}$ and $\phi \in C_{\gamma}(H)$, the continuity of the $C_{\gamma}(H)$-valued function $\tau \mapsto$ $U(t, \tau) \phi$ on $(-\infty, t]$ is not a clear fact (see Lemma 4.4 and the auxiliary lemmas). There are some essential differences between the autonomous dynamical system and non-autonomous one, say the continuous dependence of the dynamical system on their parameters. Consider a continuous process $\{S(t, \tau)\}_{t \geqslant \tau}$ on some Banach space $X$. Even if we have known that the $X$-valued function $t \mapsto S(t, \tau) x_{0}$ is continuous on $\mathbb{R}$ for every given $\tau$ and $x_{0} \in X$, the convergence $\left\|S\left(t_{*}, \tau\right) x_{0}-x_{0}\right\|_{X} \rightarrow 0$ as $\tau \rightarrow t_{*}^{-}$may still depend on $\tau$. In fact, when $\tau \rightarrow t_{*}^{-}, S\left(t_{*}, \tau\right) x_{0}$ changes simultaneously with different initial times $\tau$. This is caused naturally by the non-autonomous phenomenon.

In the end of this article, we want to propose a problem, saying, how to formulate reasonably and construct the statistical solutions for the evolution system containing delays. As for the 3D GMNS equations discussed here, we have constructed the invariant measures on the phase space $C_{\gamma}(H)$. However, we seem unable to construct its statistical solutions via the invariant measures and pullback attractors, as done in [44-47], because the invariant measures are contained in the phase space $C_{\gamma}(H)$ while the equation (2.9) is interpreted in the $\mathcal{D}\left(\tau,+\infty ; V^{\prime}\right)$ sense.

\section{Acknowledgement}

This work was supported by NSF of China with No.11801190, 11971356 and by NSF of Zhejiang Province with No.LY17A010011, and also supported by FEDER and the Spanish Ministerio de Ciencia, Innovación y Universidades project PGC2018-096540-B-I00. 


\section{References}

[1] J. P. Aubin, A. Cellina, Differential Inclusions, Springer-Verlag, New York, 1984.

[2] T. Caraballo, J. Real, Navier-Stokes equations with delays, R. Soc. Lond. Proc. Ser. A Math. Phys. Eng. Sci., 457 (2001), 2441-2453.

[3] T. Caraballo, J. Real, Asymptotic behaviour of two-dimensional Navier-Stokes equations with delays, R. Soc. Lond. Proc. Ser. A Math. Phys. Eng. Sci., 459 (2003), 3181-3194.

[4] T. Caraballo, J. Real, Attractors for 2D Navier-Stokes models with delays, J. Differential Equations, 205 (2004), 270-296.

[5] T. Caraballo, J. Real, P. E. Kloeden, Unique strong solutions and $V$-attractors of a three dimensional system of globally modified Navier-Stokes equations, Adv. Nonlinear Study, 6 (2006), 411-436.

[6] T. Caraballo, J. Real, P. E. Kloeden, Addendum to the paper "Unique strong solutions and $V$ attractors of a 3-dimensional system of globally modified Navier-Stokes equations, Advanced Nonlinear Studies 6 (2006), 411-436. Adv. Nonlinear Study, 10 (2006), 245-247.

[7] T. Caraballo, P. Marín-Rubio, J. Valero, Attractors for differential equations with unbounded delays, J. Differential Equations, 239 (2007), 311-342.

[8] T. Caraballo, P. E. Kloeden, J. Real, Invariant measures and statistical solutions of the globally modified Navier-Stokes equations, Discrete Cont. Dyn. Syst.-B, 10 (2008), 761-781.

[9] T. Caraballo, J. Real, A. M. Márquez-Durán, Three-dimensional system of globally modified NavierStokes equations with delay, Inter. J. Bifur. Chaos, 20 (2010), 2869-2883.

[10] A. N. Carvalho, J. A. Langa, J. C. Robinson, Attractors of Infinite Dimensional Nonautonomous Dynamical Systems, Applied Mathematical Sciences Vol. 182, Springer, New York, 2013.

[11] S. G. Chefranov, A. S. Chefranov, The new analytical solution of the 3D Navier-Stokes equation for compressible medium clarifies the sixth Millennium Prize problem, Cardiometry, (2017), 18-33.

[12] S. G. Chefranov, A. S. Chefranov, The new exact solution of the compressible 3D Navier-Stokes equations, Comm. Nonl. Sci. Numer. Simul., 83 (2020), 105118.

[13] M. Chekroun, N. E. Glatt-Holtz, Invariant measures for dissipative dynamical systems: Abstract results and applications, Comm. Math. Phys., 316 (2012), 723-761.

[14] S. Das, P. Das, P. Das, Dynamics and control of multidrug-resistant bacterial infection in hospital with multiple delays, Comm. Nonl. Sci. Numer. Simul., 89 (2020), 105279.

[15] B. Dong, J. Song, Globally regularity and asymptotic behavior of modified Navier-Stokes equations with fractional dissipation, Discrete Cont. Dyn. Syst., 32 (2012), 57-79.

[16] C. Foias, O. Manley, R. Rosa, R. Temam, Navier-Stokes Equations and Turbulence, Cambridge University Press, Cambridge, 2001.

[17] R. Garrappa, E. Kaslik, On initial conditions for fractional delay differential equations, Comm. Nonl. Sci. Numer. Simul., 90 (2020), 105359.

[18] M. J. Garrido-Atienza, P. Marín-Rubio, Navier-Stokes equations with delays on unbounded domains, Nonlinear Anal. 64 (2006), 1100-1118.

[19] J. García-Luengo, P. Marín-Rubio, J. Real, Pullback attractors in $V$ for non-autonomous 2D-NavierStokes equations and their tempered behavior, J. Differential Equations, 252(2012), 4333-4356.

[20] S. M. Guzzo, G. Planas, Existence of solutions for a class of Navier-Stokes equations with infinite delay, Appl. Analysis, 94 (2015), 840-855. 
[21] P. E. Kloeden, J. A. Langa, J. Real, Pullback $V$-attractors of a three dimensional system of nonautonomous globally modified Navier-Stokes equations: Existence and finite fractal dimension, Comm. Pure Appl. Anal., 6 (2007), 937-955.

[22] P. E. Kloeden, P. Marín-Rubio, J. Real, Equivalence of invariant measures and stationary statistical solutions for the autonomous globally modified Navier-Stokes equations, Comm. Pure Appl. Anal., 8 (2009), 785-802.

[23] P. E. Kloeden, J. Valero, The weak connectedness of the attainability set of weak solutions of the 3D Navier-Stokes equations, Proc. Roy. Soc. London A, 463 (2007), 1491-1508.

[24] X. Li, W. Shen, C. Sun, Invariant measures for complex-valued dissipative dynamical systems and applications, Discrete Cont. Dyn. Syst.-B, 22 (2017), 2427-2446.

[25] L. Liu, T. Caraballo, P. Marín-Rubio, Stability results for 2D Navier-Stokes equations with unbounded delay, J. Differential Equations, 265 (2018), 5685-5708.

[26] G. Łukaszewicz, Pullback attractors and statistical solutions for 2-D Navier-Stokes equations, Discrete Cont. Dyn. Syst.-B, 9 (2008), 643-659.

[27] G. Łukaszewicz, J. Real, J. C. Robinson, Invariant measures for dissipative dynamical systems and generalised Banach limits, J. Dyn. Differential Equations, 23 (2011), 225-250.

[28] G. Łukaszewicz, J. C. Robinson, Invariant measures for non-autonomous dissipative dynamical systems, Discrete Cont. Dyn. Syst., 34 (2014), 4211-4222.

[29] P. Marín-Rubio, J. Real, Attractors for 2D-Navier-Stokes equations with delays on some unbounded domains, Nonlinear Anal., 67 (2007), 2784-2799.

[30] P. Marín-Rubio, A. M. Márquez-Durán, J. Real, Three dimensional system of globally modified Navier-Stokes equations with infinite delays, Discrete Cont. Dyn. Syst.-B, 14 (2010), 655-673.

[31] P. Marín-Rubio, A. M. Márquez-Durán, J. Real, Pullback attractors for globally modified NavierStokes equations with infinite delays, Discrete Cont. Dyn. Syst., 31 (2011), 779-796.

[32] P. Marín-Rubio, J. Real, J. Valero, Pullback attractors for a two-dimensional Navier-Stokes model in an infinite delay case, Nonlinear Anal., 74 (2011), 2012-2030.

[33] P. Marín-Rubio, J. Real, A. M. Márquez-Durán, On the convergence of solutions of globally modified Navier-Stokes equations with delays to solutions of Navier-Stokes equations with delays, $A d v$. Nonlinear Study, 11 (2011), 917-927.

[34] P. Marín-Rubio, A. M. Márquez-Durán, J. Real, Asymptotic behavior of solutions for a three dimensional system of globally modified Navier-Stokes equations with a locally Lipschitz delay term, Nonlinear Anal., 79 (2013), 68-79.

[35] A. M. Márquez-Durán, Existence and uniqueness of solutions, and pullback attractor for a system of globally modified 3D-Navier-Stokes equations with finite delay, SeMA Journal, 51 (2010), 117-124.

[36] C. B. G. Moussa, Invariant measures for multivalued semigroups, J. Math. Anal. Appl., 455 (2017), 1234-1248.

[37] M. Romito, The uniqueness of weak solutions of the globally modified Navier-Stokes equations, Adv. Nonlinear Study, 9 (2009), 425-427.

[38] G. R. Sell and Y. You, Dynamics of Evolutionary Equations. Springer, 2002.

[39] R. Temam, Navier-Stokes equations, Theory and Numerical Analysis, 2nd ed., North Holland, Amsterdam, 1979. 
[40] X. Wang, Upper-semicontinuity of stationary statistical properties of dissipative systems, Discrete Cont. Dyn. Syst., 23 (2009), 521-540.

[41] C. Zhao, L. Yang, Pullback attractor and invariant measures for the globally modified Navier-Stokes equations, Comm. Math. Sci., 15 (2017), 1565-1580.

[42] C. Zhao, G. Xue, G. Łukaszewicz, Pulback attractor and invariant measures for discrete KleinGordon-Schrödinger equations, Discrete Cont. Dyn. Syst.-B, 23 (2018), 4021-4044.

[43] C. Zhao, T. Caraballo, Asymptotic regularity of trajectory attractor and trajectory statistical solution for the 3D globally modified Navier-Stokes equations, J. Differential Equations, 266 (2019), $7205-$ 7229.

[44] C. Zhao, Y. Li, T. Caraballo, Trajectory statistical solutions and Liouville type equations for evolution equations: Abstract results and applications, J. Differential Equations, 269(2020), 467-494.

[45] C. Zhao, Y. Li, Y. Sang, Using trajectory attractor to construct trajectory statistical solutions for 3D incompressible micropolar flows, Z. Angew. Math. Mech., 100(2020), e201800197.

[46] C. Zhao, Z. Song, T. Caraballo, Strong trajectory statistical solutions and Liouville type equations for dissipative Euler equations, Appl. Math. Lett., 99 (2020), 105981.

[47] C. Zhao, Y. Li, Z. Song, Trajectory statistical solutions for the 3D Navier-Stokes equations: The trajectory attractor approach, Nonlinear Anal.-RWA, 53(2020), 103077.

[48] Z. Zhu, C. Zhao, Pullback attractor and invariant measures for the three-dimensional regularized MHD equations, Discrete Cont. Dyn. Syst., 38 (2018), 1461-1477. 


\title{
Invariant measures for the 3D globally modified Navier-Stokes equations with unbounded variable delays
}

\author{
Jintao Wang ${ }^{a *}, \quad$ Caidi Zhao ${ }^{b \dagger}, \quad$ Tomás Caraballo ${ }^{c \ddagger}$ \\ ${ }^{a}$ Center for Mathematical Sciences, Huazhong University of Science and Technology, \\ Wuhan, Hubei Province, 430074, People's Republic of China \\ ${ }^{b}$ Department of Mathematics, Wenzhou University, \\ Wenzhou, Zhejiang Province, 325035, People's Republic of China \\ ${ }^{c}$ Departmento de Ecuaciones Diferenciales y Análisis Numérico, Facultad de Matemáticas, \\ Universidad de Sevilla, c/Tarfia s/n, 41012-Sevilla, Spain
}

July 7, 2020

\begin{abstract}
This article investigates the three-dimensional globally modified Navier-Stokes equations with unbounded variable delays. Firstly, we prove the global well-posedness of the solutions, and give the existence of the pullback attractor for the associated process. Then, we construct a family of invariant Borel probability measures, which is supported by the pullback attractor.
\end{abstract}

Keywords: Globally modified Navier-Stokes equations; Unbounded variable delays; Invariant measures; Pullback attractors.

MSC2010: 35B41, 34D35, 76F20

\section{Introduction}

In this article, we study the following three-dimensional (3D) system of globally modified NavierStokes (GMNS for short) equations with unbounded variable delays

$$
\begin{cases}\frac{\partial u}{\partial t}-\nu \Delta u+F_{N}\left(\|u\|_{V}\right)[(u \cdot \nabla) u]+\nabla p=f(x, t)+g(t, u(t-\rho(t))) & \text { in }(\tau,+\infty) \times \Omega, \\ \nabla \cdot u=0 & \text { in }(\tau,+\infty) \times \Omega, \\ u=0 & \text { on }(\tau,+\infty) \times \partial \Omega, \\ u(\tau+s, x)=\phi(s, x) & \text { in }(-\infty, 0] \times \Omega,\end{cases}
$$

where the unknown functions $u=u(x, t)$ and $p=p(x, t)$ denote, respectively, the velocity field of the fluid and the pressure, the positive constant $\nu$ is the viscosity coefficient, $f(x, t)$ is the density of the

\footnotetext{
*E-mail: wangjt@hust.edu.cn

${ }^{\dagger}$ Corresponding author, E-mail: zhaocaidi2013@163.com or zhaocaidi@wzu.edu.cn

${ }^{\ddagger}$ E-mail: caraball@us.es
} 
volume forces, $g$ is the external force affected by memory during the time range $(-\infty, \tau], \rho: \mathbb{R} \rightarrow \mathbb{R}^{+}$ is a delay function satisfying $\rho \in L_{\mathrm{loc}}^{\infty}(\mathbb{R})$ and $\phi$ is the initial datum on the interval $(-\infty, 0]$. Here $\|u\|_{V}=\|\nabla u\|_{\left(L^{2}(\Omega)\right)^{3}}$ and the function $F_{N}(\cdot):(0,+\infty) \mapsto(0,1]$ is defined as

$$
F_{N}(r):=\min \{1, N / r\}, \quad r \in(0,+\infty),
$$

where $N$ is a positive integer. In addition, $\Omega \subset \mathbb{R}^{3}$ is an open bounded set with the smooth boundary $\partial \Omega$.

The GMNS equations with $g \equiv 0$ (that is without delays) was formulated in [5]. It is one kind of global modifications of the Navier-Stokes (NS for short) equations with the modifying factor $F_{N}\left(\|u\|_{V}\right)$ depending on the $\left(L^{2}(\Omega)\right)^{3}$-norm of $\nabla u$. This modification brings some good properties to the solutions: global existence, uniqueness and regularity, in comparison with the multiple-solution property of NS equations (see $[11,12,16]$ and the references therein for readers' perspective). The GMNS equations are useful in obtaining some new results for the 3D NS equations. For instance, it was used in [5] to establish the existence of bounded entire weak solutions for the 3D NS equations, and in [23], the authors applied it to show weak compactness and weak connectedness of the attainability set of the weak solutions of the 3D NS equations satisfying an energy inequality. At the same time, it was proved in $[5,33]$ that the solutions of the GMNS equations converge to those of the 3D NS equations in some proper sense. Therefore, the GMNS equations (1.1) with $g \equiv 0$ can be regarded as an approximation to the 3D NS equations.

The GMNS equations (1.1) with $g \equiv 0$ have been extensively studied. For example, one can see $[5,6,15,21,37]$ for the existence and uniqueness of the weak and strong solutions, $[8,22,28,41]$ for invariant measure and statistical solutions, [43] for the existence of trajectory attractor and trajectory statistical solutions. However, the real world is inundated with many situations in which the model is better described with some terms containing delays appearing in the equations. These delays may appear, for instance, when the current behavior is influenced by its previous states, or one wants to control the system by imposing an external force which depends upon both the present state and the history of the solutions. The delayed partial differential equations are usually used to describe these delayed phenomena ( $[14,17])$.

Nowadays, delayed partial differential equations have drawn much attention and have been extensively studied. For example, the existence and asymptotic behaviors of solutions for the NS equations with finite delays were investigated in $[2-4,18,29]$; the existence of pullback attractors for the NS equations with finite or infinite delays was studied in [7,20,25,32]. Some known references concerning the delayed 3D GMNS equations are summarized as:

$1^{\circ}$ Finite delays: the existence, uniqueness and global asymptotic exponentially stability of the stationary solution, as well as the convergence of solutions to those of the NS equations were established in $[33,34]$.

$2^{\circ}$ Infinite delays: the well-posedness and global exponential decay of the solutions to the stationary solution, as well as the existence of pullback attractor were investigated in [30,31].

$3^{\circ}$ Bounded variable delays: the existence and uniqueness of solutions, as well as the existence of pullback attractors were proved in $[9,35]$.

However, up to our knowledge, there is no reference concerning the 3D GMNS equations with unbounded variable delays. 
The motivation of this article is to investigate the existence of invariant Borel probability measures for the 3D GMNS equations with unbounded variable delays. The invariant measures and statistical solutions have played a significant role and developed into a crucial tool in the research of turbulence (see Foias et al. [16]) in recent decades. This is mainly due to the fact that some time-average quantities essentially measure several important aspects of turbulent flows. The invariant measures and statistical properties of dissipative systems were studied by a number of researchers after that. For instance, Wang considered the upper semi-continuity of stationary statistical properties for dissipative systems in [40]. Łukaszewicz, Real and Robinson in [27] constructed the invariant measures for general continuous dynamical systems on metric spaces by using the generalized Banach limit. For a much wider class of dissipative semigroups, Chekroun and Glatt-Holtz [13] also applied the generalized Banach limit to constructing the invariant measures, but they generalized and simplified the proofs of [27] and [40].

Recently, a series of works developed some techniques to provide a construction of invariant measures for non-autonomous systems with minimal assumptions on the underlying dynamical process (see Foias et al. [16], Wang [40] and Łukaszewicz et al. [26-28]). Nowadays, these theories have been employed to establish invariant measures for some evolution equations, (see e.g. [24, 36, 41, 48] and the references therein). However, as far as we know, there is no reference investigating the invariant measures for delayed evolution equations.

The main result of the current article is the existence of invariant Borel probability measures for the 3D GMNS equations with unbounded variable delays. We will use the abstract theory for dissipative non-autonomous system in [28, Theorem 3.1]. To this end, we shall prove that the solution operators associated to the problem (1.1) generate a continuous process $\{U(t, \tau)\}_{t \geqslant \tau}$ in the phase space $\mathcal{C}_{\gamma}(H)$ (see notation in Section 2) and

(1) the process $\{U(t, \tau)\}_{t \geqslant \tau}$ is pullback strongly bounded in $\mathcal{C}_{\gamma}(H)$;

(2) the process $\{U(t, \tau)\}_{t \geqslant \tau}$ is pullback asymptotically compact in $\mathcal{C}_{\gamma}(H)$;

(3) for each given $t_{*} \in \mathbb{R}$ and $\phi_{*} \in \mathcal{C}_{\gamma}(H)$, the $\mathcal{C}_{\gamma}(H)$-valued function $\tau \mapsto U\left(t_{*}, \tau\right) \phi_{*}$ is continuous and bounded on $(-\infty, t]$ (this is called $\tau$-continuity of the process $\{U(t, \tau)\}_{t \geqslant \tau}$ ).

We first use a Galerkin approximation argument, and some a priori estimates, to prove the global well-posedness of the solutions to the problem (1.1). Also we do some estimates of the solutions to obtain the existence of strongly bounded pullback absorbing sets. Secondly we apply the Ascoli-Arzelà type theorem ( [1]) to establish the pullback asymptotic compactness of the process $\{U(t, \tau)\}_{t \geqslant \tau}$. This is prompted by the delay appearing in the equations. Since the problem discussed contains unbounded variable delays, it seems suitable for us to choose $C_{\gamma}(H)$ as the phase space. It has been shown that the Ascoli-Arzelà theorem is a powerful tool to investigate the compactness of subsets of $C_{\gamma}(H)$. Thirdly, for each given $t_{*} \in \mathbb{R}$ and $\phi_{*} \in C_{\gamma}(H)$, the continuity of the $C_{\gamma}(H)$-valued function $\tau \mapsto U\left(t_{*}, \tau\right) \phi_{*}$ on $(-\infty, t]$ is not a clear fact (see Lemma 4.4 and the auxiliary lemmas). There are some essential differences between the autonomous dynamical system and non-autonomous one, say the continuous dependence of the dynamical system on their parameters. Even if we have known that the $C_{\gamma}(H)$ valued function $t \mapsto U(t, \tau) \phi_{*}$ is continuous on $\mathbb{R}$ for every given $\tau$ and $\phi_{*} \in C_{\gamma}(H)$, the convergence $\left\|U\left(t_{*}, \tau\right) \phi_{*}-\phi_{*}\right\|_{\gamma} \rightarrow 0$ as $\tau \rightarrow t_{*}^{-}$may still depend on $\tau$ itself. In fact, when $\tau \rightarrow t_{*}^{-}, U\left(t_{*}, \tau\right) \phi_{*}$ also changes with different initial times $\tau$. This is caused naturally by the non-autonomous and delayed phenomena. We will take advantage of the structure of the GMNS equations to prove the $\tau$-continuity of 
the process $\{U(t, \tau)\}_{t \geqslant \tau}$.

This article is organized as follows. In the next section, we first introduce some notations and operators, and then prove the global well-posedness of solutions to the problem (1.1). Section 3 is devoted to the proof of the existence of pullback attractors. In Section 4, we first prove the $\tau$-continuity of the process and then construct a family of invariant Borel probability measures which is supported by the pullback attractors. We end the article with conclusions and remarks in Section 5.

\section{Global well-posedness of solutions}

In this section, we first introduce some notations and operators. Then we prove the global wellposedness of the problem (1.1).

In this article, we use the following notations:

$\mathbb{L}^{2}(\Omega)=\left(L^{2}(\Omega)\right)^{3}$-the 3D Lebesgue space with norm $\|\cdot\|_{\mathbb{L}^{2}(\Omega)}=\|\cdot\|$;

$\mathbb{H}^{1}(\Omega)=$ the $3 \mathrm{D}$ Sobolev space $\left\{\phi \in \mathbb{L}^{2}(\Omega) \mid \nabla \phi \in \mathbb{L}^{2}(\Omega)\right\}$ with norm $\|\nabla \cdot\|_{\mathbb{L}^{2}(\Omega)}$;

$\mathbb{H}_{0}^{1}(\Omega)=$ closure of $\left(\mathcal{C}_{0}^{\infty}(\Omega)\right)^{3}$ in $\mathbb{H}^{1}(\Omega)$ with norm $\|\cdot\|_{\mathbb{H}^{1}(\Omega)}$;

$\mathcal{V}=\left\{\phi \in\left(\mathcal{C}_{0}^{\infty}(\Omega)\right)^{3} \mid \nabla \cdot \phi=0\right\}$

$H=$ closure of $\mathcal{V}$ in $\mathbb{L}^{2}(\Omega)$ with norm $\|\cdot\|$ and inner product $(\cdot, \cdot)$;

$V=$ closure of $\mathcal{V}$ in $\mathbb{H}^{1}(\Omega)$ with norm $\|\cdot\|_{V}=\|\nabla \cdot\|_{\mathbb{L}^{2}(\Omega)}$;

$V^{\prime}=$ dual space of $V$ with norm $\|\cdot\|_{V^{\prime}} ;\langle\cdot, \cdot\rangle$ - the dual pairing between $V$ and $V^{\prime}$;

In addition, we will use the notation $a \lesssim b$ to mean that $a \leqslant c b$ for a universal constant $c>0$ that only depends on the parameters coming from the problem and will not result in confusion.

For short, we will write the problem (1.1) in a functional form. To this end, we introduce some classical operators which are usually used to handle the 3D incompressible NS and GMNS equations. At the same time, we will select some known estimates and properties (see e.g. [39]) of these operators.

We first consider the operator $A: V \rightarrow V^{\prime}$ defined as

$$
\langle A u, v\rangle=(\nabla u, \nabla v), \quad \forall u, v \in V,
$$

where $D(A)=\mathbb{H}^{2}(\Omega) \cap V$ and $\mathbb{H}^{2}(\Omega)=\left\{\phi \in \mathbb{H}^{1}(\Omega) \mid \Delta \phi \in \mathbb{L}^{2}(\Omega)\right\}$. In fact, for each $u \in D(A)$, $A u=-P \Delta u$, and hence $A$ is the Stokes operator, where $P$ is the Leray-Helmholtz projection from $\mathbb{L}^{2}(\Omega)$ onto $H$.

Secondly, we define a trilinear form $b(\cdot, \cdot, \cdot)$ as follows,

$$
b(u, v, w)=\sum_{i, j=1}^{3} \int_{\Omega} u_{i} \frac{\partial v_{j}}{\partial x_{i}} w_{j} \mathrm{~d} x, \quad \forall u, v, w \in \mathbb{H}_{0}^{1}(\Omega) .
$$

Notice that $V$ is a closed subspace of $\mathbb{H}_{0}^{1}(\Omega)$. One can check that $b(u, v, w)$ is continuous on $V \times V \times V$, and $b(u, v, w)=-b(u, w, v), b(u, v, v)=0, \forall u, v, w \in V$. For each $u, v \in V$,

$$
\langle B(u, v), w\rangle=b(u, v, w), \quad \forall w \in V,
$$

defines a continuous function $B(u, v)$ on $V \times V$. At the same time, we have (cf. [39])

$$
\begin{array}{ll}
\|b(u, v, w)\| \lesssim\|u\|_{V}^{1 / 2}\|A u\|^{1 / 2}\|v\|_{V}\|w\|, & \forall u \in D(A), v \in V, w \in H \\
\|b(u, v, w)\| \lesssim\|u\|_{V}\|v\|_{V}\|w\|_{V}^{1 / 2}\|w\|^{1 / 2}, & \forall u, v, w \in V .
\end{array}
$$


We further set

$$
\begin{aligned}
& b_{N}(u, v, w)=F_{N}\left(\|v\|_{V}\right) b(u, v, w), \quad \forall u, v, w \in V, \\
& \left\langle B_{N}(u, v), w\right\rangle=b_{N}(u, v, w), \quad \forall u, v, w \in V .
\end{aligned}
$$

Then $b_{N}(u, v, w)$ is linear in $u$ and $w$, but not linear in $v$. However, we still have (see [37])

$$
\begin{aligned}
b_{N}(u, v, v) & =0, \quad \forall u, v \in V, \\
\left|b_{N}(u, v, w)\right| & \lesssim N\|u\|_{V}\|w\|_{V}, \quad \forall u, v, w \in V, \\
\left|b_{N}(u, v, w)\right| & \lesssim N\|A u\|\|w\|, \quad \forall u \in D(A), v \in V, w \in H .
\end{aligned}
$$

Furthermore, for every $u, v \in V$ and each $N>0$,

$$
\begin{aligned}
0 & \leqslant\|u\|_{V} F_{N}\left(\|u\|_{V}\right) \leqslant N, \\
\left|F_{N}\left(\|u\|_{V}\right)-F_{N}\left(\|v\|_{V}\right)\right| & \leqslant \frac{1}{N} F_{N}\left(\|u\|_{V}\right) F\left(\|v\|_{V}\right)\|u-v\|_{V} .
\end{aligned}
$$

To formulate the problem (1.1) in a proper manner, we require that the functions $\rho(t), f(x, t)$ and $g(t, u(t-\rho(t)))$ in (1.1) satisfy some natural hypotheses.

(H1) Suppose that the function $\rho: \mathbb{R} \mapsto \mathbb{R}_{+}$belongs to $L_{\mathrm{loc}}^{\infty}(\mathbb{R})$ and $f(t, x) \in L_{\mathrm{loc}}^{2}\left(\mathbb{R} ; \mathbb{L}^{2}(\Omega)\right)$. Also assume that $g: \mathbb{R} \times H \rightarrow H$ satisfies

(g1) for every $u \in H$, the mapping $\mathbb{R} \ni t \mapsto g(t, u)$ is measurable;

(g2) $g(t, 0)=0$ for all $t \in \mathbb{R}$;

(g3) there exists a positive constant $\gamma$ and a nonnegative function $L_{g}: \mathbb{R} \rightarrow \mathbb{R}_{+}$such that

$$
L_{g}(\cdot) e^{\gamma \rho(\cdot)} \in L_{\mathrm{loc}}^{2}(\mathbb{R}) \quad \text { and } \quad\|g(t, u)-g(t, v)\| \leqslant L_{g}(t)\|u-v\|, \quad \forall t \in \mathbb{R}, \forall u, v \in H .
$$

Remark 2.1. In Remark 3.1 we will give a concrete example to show the existence of the functions $\rho(t)$, $f(t, x), g(t, u), L_{g}(t)$ and the constant $\gamma$ that satisfy our assumption (H1). In the sequel, the constant $\gamma$ is from $(g 3)$.

In order to deal with the unbounded variable delays, we consider the space

$$
C_{\gamma}(H):=\left\{\phi \in C((-\infty, 0] ; H): \sup _{s \leqslant 0} e^{\gamma s}\|\phi(s)\|<+\infty, \exists \lim _{s \rightarrow-\infty} e^{\gamma s} \phi(s) \in H\right\},
$$

with the norm $\|\phi\|_{\gamma}:=\sup _{s \leqslant 0} e^{\gamma s}\|\phi(s)\|$. Then $\left(C_{\gamma}(H),\|\cdot\|_{\gamma}\right)$ is a Banach space. In addition, for each $\tau \in \mathbb{R}$ we denote conventionally $u_{\tau}(s)=u(\tau+s)$ with $s \in(-\infty, 0]$.

We now specify the definition of the weak solution to the problem (1.1).

Definition 2.1. Let $\tau \in \mathbb{R}$ and $\phi \in C_{\gamma}(H)$ be given. If for all $T>\tau$, a function $u \in C((-\infty, T], H) \cap$ $L^{2}(\tau, T ; V)$ with $u_{\tau}=\phi$ satisfies

$$
\frac{\mathrm{d} u(t)}{\mathrm{d} t}+\nu A u(t)+B_{N}(u(t), u(t))=f(t)+g(t, u(t-\rho(t)),
$$

in the sense of distribution $\mathcal{D}^{\prime}\left(\tau,+\infty ; V^{\prime}\right)$, then we call $u$ a weak solution of the problem (1.1) corresponding to the initial datum $\phi$. 
For the existence and uniqueness of solutions to the problem (1.1), we have the following result.

Theorem 2.1. Let the assumption (H1) hold true. Then for each $\tau \in \mathbb{R}$ and $\phi \in C_{\gamma}(H)$, there corresponds a unique weak solution $u(\cdot)=u(\cdot ; \tau, \phi)$ to the problem (1.1), and $u$ is in fact a strong solution in the sense that

$$
u \in C((\tau, T], V) \cap L^{2}(\tau+\varepsilon, T ; D(A)), \quad \forall \varepsilon>0, \quad \forall T>\tau+\varepsilon .
$$

Moreover, if $\phi(0) \in V$, then

$$
u \in C([\tau, T] ; V) \cap L^{2}(\tau, T ; D(A)), \quad \forall T>\tau .
$$

Proof. The proof is similar to that of [30, Theorem 2] and [31, Theorem 1] with the unbounded variable delays in place of the infinite delays. Following the proof of [30, Theorem 2] with some different estimates, we present the parts that will be used in the sequel of this article.

For the Galerkin approximation solution $u^{(m)}$, we have

$$
\begin{aligned}
\frac{\mathrm{d}}{\mathrm{d} t}\left\|u^{(m)}(t)\right\|^{2}+2 \nu\left\|u^{(m)}(t)\right\|_{V}^{2} & =2\left(f(t), u^{(m)}(t)\right)+2\left(g\left(t, u^{(m)}(t-\rho(t))\right), u^{(m)}(t)\right) \\
& \lesssim \nu\left\|u^{(m)}(t)\right\|_{V}^{2}+\|f(t)\|^{2}+2 L_{g}(t)\left\|u^{(m)}(t-\rho(t))\right\|\left\|u^{(m)}(t)\right\| \\
& \lesssim \nu\left\|u^{(m)}(t)\right\|_{V}^{2}+\|f(t)\|^{2}+2 L_{g}(t) e^{\gamma \rho(t)}\left\|u_{t}^{m}\right\|_{\gamma}^{2},
\end{aligned}
$$

using the facts that $\left\|u^{(m)}(t)\right\|=\left\|u_{t}^{(m)}(0)\right\| \leqslant\left\|u_{t}^{(m)}\right\|_{\gamma}$ and

$$
\left\|u^{(m)}(t-\rho(t))\right\|=e^{\gamma \rho(t)} e^{-\gamma \rho(t)}\left\|u^{(m)}(t-\rho(t))\right\| \leqslant e^{\gamma \rho(t)}\left\|u_{t}^{(m)}\right\|_{\gamma} .
$$

Hence, for all $t \geqslant \tau$ there holds

$$
\left\|u^{(m)}(t)\right\|^{2}+\nu \int_{\tau}^{t}\left\|u^{(m)}(s)\right\|_{V}^{2} \mathrm{~d} s \lesssim\left\|u^{(m)}(\tau)\right\|^{2}+\int_{\tau}^{t}\left(\|f(s)\|^{2}+2 L_{g}(s) e^{\gamma \rho(s)}\left\|u_{s}^{(m)}\right\|_{\gamma}^{2}\right) \mathrm{d} s .
$$

By (2.13) and the definition of the norm $\left\|u_{t}^{(m)}\right\|_{\gamma}^{2}$, we have

$$
\begin{aligned}
\left\|u_{t}^{(m)}\right\|_{\gamma}^{2} & \lesssim \max \left\{\sup _{\theta \leqslant \tau-t} e^{2 \gamma \theta}\|\phi(\theta+t-\tau)\|^{2}\right. \\
& \left.\sup _{\theta \in[\tau-t, 0]}\left(e^{2 \gamma \theta}\|u(\tau)\|^{2}+e^{2 \gamma \theta} \int_{\tau}^{t+\theta}\left(\|f(s)\|^{2}+2 L_{g}(s) e^{\gamma \rho(s)}\left\|u_{s}^{(m)}\right\|_{\gamma}^{2}\right) \mathrm{d} s\right)\right\} \\
& \lesssim \max \left\{\sup _{\theta \leqslant 0} e^{2 \gamma(\theta+\tau-t)}\|\phi(\theta)\|^{2},\|u(\tau)\|^{2}+\int_{\tau}^{t}\left(\|f(s)\|^{2}+2 L_{g}(s) e^{\gamma \rho(s)}\left\|u_{s}^{(m)}\right\|_{\gamma}^{2}\right) \mathrm{d} s\right\} \\
& \lesssim\|\phi\|_{\gamma}^{2}+\int_{\tau}^{t}\left(\|f(s)\|^{2}+2 L_{g}(s) e^{\gamma \rho(s)}\left\|u_{s}^{(m)}\right\|_{\gamma}^{2}\right) \mathrm{d} s .
\end{aligned}
$$

Applying Gronwall's lemma to (2.14) gives

$$
\left\|u_{t}^{(m)}\right\|_{\gamma}^{2} \lesssim\left(\|\phi\|_{\gamma}^{2}+\int_{\tau}^{t}\|f(s)\|^{2} \mathrm{~d} s\right) \exp \left(2 \int_{\tau}^{t} L_{g}(s) e^{\gamma \rho(s)} \mathrm{d} s\right)
$$


We then conclude from (2.13) and (2.15) that, with the assumption (H1) in hand,

$$
\begin{aligned}
& \left\|u_{t}^{(m)}\right\|_{\gamma}^{2}+\left\|u^{(m)}\right\|_{L^{2}(\tau, T ; V)}^{2} \lesssim 1, \quad \forall t \in[\tau, T], \forall\|\phi\|_{\gamma} \leqslant R, \forall m \geqslant 1, \\
& \int_{\tau}^{T}\left\|g\left(s, u^{(m)}(s-\rho(s))\right)\right\|^{2} \mathrm{~d} s \leqslant \int_{\tau}^{T}\left(L_{g}(s) e^{\gamma \rho(s)}\right)^{2}\left\|u_{s}^{(m)}\right\|_{\gamma}^{2} \mathrm{~d} s \lesssim\left\|L_{g} e^{\gamma \rho}\right\|_{L^{2}(\tau, T)}^{2} .
\end{aligned}
$$

Estimate (2.16) implies that

$$
g\left(\cdot, u^{(m)}(\cdot-\rho(\cdot))\right) \in L^{2}\left(\tau, T ; \mathbb{L}^{2}(\Omega)\right) .
$$

The rest tasks are to prove that the Galerkin approximate solutions $\left\{u^{(m)}\right\}$ possess a convergent subsequence and its limiting function is the solution satisfying Theorem 2.1. The procedures are similar to those of [30, Theorem 2] and we omit the details here.

The result below shows that the solutions to (1.1) depend continuously on the initial data.

Theorem 2.2. Let the assumption (H1) hold. Let $u=u(\cdot ; \tau, \phi)$ and $\tilde{u}=\tilde{u}(\cdot ; \tau, \tilde{\phi})$ be two solutions of the problem (1.1) with the initial data $\phi$ and $\tilde{\phi}$ at the initial time $\tau$, respectively. Then we have

$$
\begin{aligned}
& \max _{\theta \in[\tau, t]}\|u(\theta)-\tilde{u}(\theta)\|^{2} \\
\lesssim & \|\phi(0)-\tilde{\phi}(0)\|^{2} \exp \left(\int_{\tau}^{t} 2 L_{g}(s) e^{\gamma \rho(s)} \mathrm{d} s+(t-\tau)\right) \\
& +\|\phi-\tilde{\phi}\|_{\gamma}^{2} \int_{\tau}^{t} L_{g}(s) e^{\gamma \rho(s)} \exp \left(\int_{\tau}^{s} 2 L_{g}(\theta) e^{\gamma \rho(\theta)} \mathrm{d} \theta+(s-\tau)\right) \mathrm{d} s .
\end{aligned}
$$

The proof of Theorem 2.2 is analogous to that of [31, Proposition 1] and we omit the details.

\section{Existence of the pullback attractors}

From Theorems 2.1 and 2.2 we can conclude that the solution operators $U(t, \tau): C_{\gamma}(H) \rightarrow C_{\gamma}(H)$ defined by

$$
U(t, \tau) \phi(s)=u_{t}(s), \quad \tau \leqslant t, \quad s \in(-\infty, 0]
$$

generate a continuous process $\{U(t, \tau)\}_{t \geqslant \tau}$ on the phase space $C_{\gamma}(H)$, where $u=u(\cdot ; \tau, \phi)$ is the solution of the problem (1.1) corresponding to the initial function $\phi$ at the initial time $\tau$. In this section, we first prove that $\{U(t, \tau)\}_{t \geqslant \tau}$ possesses a bounded pullback absorbing set and is pullback asymptotically compact in $C_{\gamma}(H)$. Then we obtain the existence of the pullback attractor. For related definitions and results concerning the evolution processes and pullback attractors, we can refer to $[4,10,38]$ and the references therein.

In the sequel, we pick $\gamma$ and some parameter $\mu$ such that

$$
0<\mu<\nu \text { and }(\nu-\mu) \lambda_{1}<\gamma
$$

where $\lambda_{1}=\inf _{v \in V \backslash\{0\}} \frac{\|v\|_{V}^{2}}{\|v\|^{2}}>0$ is the first eigenvalue of the Stokes operator $A$. To obtain the existence of the bounded pullback absorbing set, we need another assumption for the functions $L_{g}(\cdot) e^{\gamma \rho(\cdot)}$ and $f$. 
(H2) Assume there is a constant $\mu$ satisfying (3.2) such that

$$
\begin{aligned}
& \underset{t \in \mathbb{R}}{\operatorname{ess} \sup } L_{g}(t) e^{\gamma \rho(t)}<(\nu-\mu) \lambda_{1} \leqslant \gamma, \\
& \int_{-\infty}^{0} e^{\sigma s}\|f(s)\|^{2} \mathrm{~d} s<+\infty
\end{aligned}
$$

hereinafter the constant $\sigma$ is given by

$$
\sigma:=2(\nu-\mu) \lambda_{1}-2 \underset{t \in \mathbb{R}}{\operatorname{ess} \sup } L_{g}(t) e^{\gamma \rho(t)} .
$$

Remark 3.1. We set

$$
\left\{\begin{array}{l}
\rho(t)=|t|, \quad t \in \mathbb{R} \\
g(t, u)=\exp \left(-e^{|t|}\right) u, \quad t \in \mathbb{R}, u \in H \\
L_{g}(t)=\exp \left(-e^{|t|}\right), \quad t \in \mathbb{R} \\
\gamma=e
\end{array}\right.
$$

then the conditions in (H1) are satisfied and

$$
\underset{t \in \mathbb{R}}{\operatorname{ess} \sup } L_{g}(t) e^{\gamma \rho(t)}=e^{\gamma \ln \gamma-\gamma}=e^{0}=1<e=\gamma .
$$

If we take $1<\nu<e$ and $\mu$ small enough, then (3.3) are satisfied. Thus, the functions and constant presented by (3.6) satisfy the conditions in (H1) and (H2). For the existence of the function $f(x, t)$ satisfying (H1) and (H2), we refer to [42, Example 3.1].

We denote by $\mathcal{P}\left(C_{\gamma}(H)\right)$ the family of all subsets of $C_{\gamma}(H)$ and consider the families of nonempty sets $\widehat{D}=\{D(t): t \in \mathbb{R}\} \subseteq \mathcal{P}\left(C_{\gamma}(H)\right)$. Let $\mathcal{D}_{\sigma}$ be the nonempty class of families $\widehat{D}=\{D(t): t \in$ $\mathbb{R}\} \subseteq \mathcal{P}\left(C_{\gamma}(H)\right)$ parameterized by time $t$ and satisfying

$$
\lim _{\tau \rightarrow-\infty}\left(e^{\sigma \tau} \sup _{v \in D(\tau)}\|v\|_{\gamma}^{2}\right)=0 .
$$

The class $\mathcal{D}_{\sigma}$ defined above is always called a tempered universe.

Lemma 3.1. Let the assumptions (H1) and (H2) hold. Then the process $\{U(t, \tau)\}_{t \geqslant \tau}$ possesses a bounded pullback absorbing set in $C_{\gamma}(H)$.

Proof. Pick some $\mu$ satisfying (3.2), and let $u=u(\cdot ; \tau, \phi)$ be the solution of the problem (1.1) corresponding to the initial function $\phi$ at the initial time $\tau$. Then we can get

$$
\frac{\mathrm{d}}{\mathrm{d} t}\|u(t)\|^{2}+2(\nu-\mu) \lambda_{1}\|u(t)\|^{2}+\mu\|u(t)\|_{V}^{2} \lesssim\|f(t)\|^{2}+2 L_{g}(t) e^{\gamma \rho(t)}\left\|u_{t}\right\|_{\gamma}^{2}, \quad t>\tau .
$$

Hence for all $t \geqslant \tau$,

$$
\begin{aligned}
& \|u(t)\|^{2}+\mu \int_{\tau}^{t} e^{-2(\nu-\mu) \lambda_{1}(t-s)}\|u(s)\|_{V}^{2} \mathrm{~d} s \\
\lesssim & e^{-2(\nu-\mu) \lambda_{1}(t-\tau)}\|u(\tau)\|^{2}+\int_{\tau}^{t} e^{-2(\nu-\mu) \lambda_{1}(t-s)}\left(\|f(s)\|^{2}+2 L_{g}(s) e^{\gamma \rho(s)}\left\|u_{s}\right\|_{\gamma}^{2}\right) \mathrm{d} s .
\end{aligned}
$$


Inequality (3.8) implies that

$$
\begin{aligned}
\left\|u_{t}\right\|_{\gamma}^{2} \lesssim \max \left\{\sup _{\theta \in(-\infty, \tau-t]} e^{2 \gamma \theta}\|\phi(\theta+t-\tau)\|^{2}, \sup _{\theta \in[\tau-t, 0]}\left(e^{2 \gamma \theta-2(\nu-\mu) \lambda_{1}(t+\tau-\theta)}\|u(\tau)\|^{2}\right.\right. \\
\left.\left.+e^{2 \gamma \theta} \int_{\tau}^{t+\theta} e^{-2(\nu-\mu) \lambda_{1}(t+\theta-s)}\left(\mid f(s)\left\|^{2}+2 L_{g}(s) e^{\gamma \rho(s)}\right\| u_{s} \|_{\gamma}^{2}\right) \mathrm{d} s\right)\right\} .
\end{aligned}
$$

By the choice of $\mu$ in (3.2), we have

$$
\begin{aligned}
& \sup _{\theta \in(-\infty, \tau-t]} e^{2 \gamma \theta}\|\phi(\theta+t-\tau)\| \leqslant e^{-(\nu-\mu) \lambda_{1}(t-\tau)}\|\phi\|_{\gamma}, \\
& \sup _{\theta \in[\tau-t, 0]} e^{2 \gamma \theta-2(\nu-\mu) \lambda_{1}(t+\tau-\theta)}\|u(\tau)\|^{2} \leqslant e^{-2(\nu-\mu) \lambda_{1}(t-\tau)}\|u(\tau)\|^{2}, \\
& \sup _{\theta \in[\tau-t, 0]} e^{2 \gamma \theta} \int_{\tau}^{t+\theta} e^{-2(\nu-\mu) \lambda_{1}(t+\theta-s)}\left(\|f(s)\|^{2}+2 L_{g}(s) e^{\gamma \rho(s)}\left\|u_{s}\right\|_{\gamma}^{2}\right) \mathrm{d} s \\
& \leqslant \int_{\tau}^{t} e^{-2(\nu-\mu) \lambda_{1}(t-s)}\left(\|f(s)\|^{2}+2 L_{g}(s) e^{\gamma \rho(s)}\left\|u_{s}\right\|_{\gamma}^{2}\right) \mathrm{d} s .
\end{aligned}
$$

It then follows from (3.9)-(3.12) that

$$
\left\|u_{t}\right\|_{\gamma}^{2} \lesssim e^{-2(\nu-\mu) \lambda_{1}(t-\tau)}\|\phi\|_{\gamma}^{2}+\int_{\tau}^{t} e^{-2(\nu-\mu) \lambda_{1}(t-s)}\left(\|f(s)\|^{2}+2 L_{g}(s) e^{\gamma \rho(s)}\left\|u_{s}\right\|_{\gamma}^{2}\right) \mathrm{d} s .
$$

Applying Gronwall's inequality to (3.13) yields

$$
\begin{aligned}
\left\|u_{t}\right\|_{\gamma}^{2} \lesssim & \|\phi\|_{\gamma}^{2} \exp \left\{-2(\nu-\mu) \lambda_{1}(t-\tau)+2 \int_{\tau}^{t} L_{g}(s) e^{\gamma \rho(s)} \mathrm{d} s\right\} \\
& +\int_{\tau}^{t}\|f(s)\|^{2} \exp \left\{-2(\nu-\mu) \lambda_{1}(t-s)+2 \int_{s}^{t} L_{g}(\sigma) \mathrm{e}^{\gamma \rho(\sigma)} \mathrm{d} \sigma\right\} \mathrm{d} s \\
& \lesssim\|\phi\|_{\gamma}^{2} e^{-\sigma(t-\tau)}+\int_{\tau}^{t}\|f(s)\|^{2} e^{-\sigma(s-\tau)} \mathrm{d} s
\end{aligned}
$$

Now we define the time-dependent family $\hat{\mathcal{B}}=\{\mathcal{B}(t): t \in \mathbb{R}\} \in \mathcal{P}\left(C_{\gamma}(H)\right)$ as

$$
\mathcal{B}(t)=\left\{v \in C_{\gamma}(H):\|v\|_{\gamma}^{2} \lesssim 1+\int_{-\infty}^{t} e^{-\sigma(t-s)}\|f(s)\|^{2} \mathrm{~d} s\right\}
$$

Then $\hat{\mathcal{B}}$ is the desired pullback $\mathcal{D}_{\sigma}$-absorbing set for $\{U(t, \tau)\}_{t \geqslant \tau}$ in $C_{\gamma}(H)$.

To establish the pullback $\mathcal{D}_{\sigma}$-asymptotic compactness for $\{U(t, \tau)\}_{t \geqslant \tau}$ in $C_{\gamma}(H)$, we will employ the generalized Ascoli-Arzelà type theorem ( [1]) to prove the following auxiliary lemma.

Lemma 3.2. Let $\left\{\tau_{n}\right\}_{n \geqslant 1}$ be a sequence such that $\tau_{n} \rightarrow-\infty$ as $n \rightarrow+\infty$ and $\tau_{n} \leqslant t_{0}$ for some fixed $t_{0} \in \mathbb{R}$, and $\left\{\phi_{n}\right\}_{n \geqslant 1}$ a sequence of functions with $\phi_{n} \in \mathcal{B}\left(\tau_{n}\right)$ for each positive integer $n$. Denote by $u^{(n)}=u^{(n)}\left(\cdot ; \tau_{n}, \phi_{n}\right)$ the solutions of the problem (1.1) corresponding to the initial data $\phi_{n}$ at the initial time $\tau_{n}$. Then there exist a subsequence (still denoted the same) $\left\{u_{t_{0}}^{(n)}\right\}_{n \geqslant 1}$ and a function $\psi \in C_{\gamma}(H)$ such that $u_{t_{0}}^{(n)} \rightarrow \psi$ in $C([-\bar{T}, 0] ; H)$ for each $\bar{T}>0$. 
Proof. Let $t_{0},\left\{\tau_{n}\right\}_{n \geqslant 1},\left\{\phi_{n}\right\}_{n \geqslant 1}$ and $u^{(n)}=u^{(n)}\left(\cdot ; \tau_{n}, \phi_{n}\right)$ be given as in this lemma. Consider two arbitrary positive numbers $\bar{T}, T$ with $T>\bar{T}$. Let $n_{0}=n_{0}\left(t_{0}, T\right)$ be the positive integer such that $\tau_{n}<t_{0}-T$ for all $n \geqslant n_{0}$. From (3.3) and (3.14)-(3.15) we deduce that for $t \in\left[t_{0}-T, t_{0}\right]$ and $n \geqslant n_{0}$,

$$
\begin{aligned}
\left\|u^{n}(t)\right\|^{2} & \lesssim R\left(t_{0}, T\right):=1+e^{-\sigma\left(t_{0}-T\right)} \int_{-\infty}^{t_{0}} e^{\sigma s}\|f(s)\|^{2} \mathrm{~d} s<+\infty \\
\left\|u_{t}^{n}\right\|_{\gamma}^{2} \lesssim R\left(t_{0}, T\right) . &
\end{aligned}
$$

We next divide two steps to establish that the conditions of the Ascoli-Arzelà type theorem are satisfied.

Step one. We first prove that

$$
\left\{u^{(n)}(t)\right\}_{n \geqslant n_{0}} \text { is precompact in } H \text { for each } t \in\left[t_{0}-\bar{T}, t_{0}\right] .
$$

By Theorem 2.1, we conclude that $u^{(n)}(t) \in D(A)$ for all $t \in\left[t_{0}-T, t_{0}\right]$, whence,

$$
\begin{aligned}
& \frac{1}{2} \frac{\mathrm{d}}{\mathrm{d} t}\left\|u^{(n)}(t)\right\|_{V}^{2}+\nu\left\|A u^{(n)}(t)\right\|^{2}+b_{N}\left(u^{(n)}(t), u^{(n)}(t), A u^{(n)}(t)\right) \\
= & \left(f(t), A u^{(n)}(t)\right)+\left(g\left(t, u^{(n)}(t-\rho(t))\right), A u^{(n)}(t)\right), \quad t \in\left[t_{0}-T, t_{0}\right] .
\end{aligned}
$$

Using Young's inequality, (2.1) and (2.6), we have

$$
\begin{aligned}
& \left(f(t), A u^{(n)}(t)\right) \lesssim \frac{\nu}{8}\left\|A u^{(n)}(t)\right\|^{2}+\|f(t)\|^{2}, \\
& \left(g\left(t, u^{(n)}(t-\rho(t))\right), A u^{(n)}(t)\right) \lesssim \frac{\nu}{8}\left\|A u^{(n)}(t)\right\|^{2}+L_{g}^{2}(t) e^{2 \gamma \rho(t)}\left\|u_{t}^{(n)}\right\|_{\gamma}^{2}, \\
& \left|b_{N}\left(u^{(n)}(t), u^{(n)}(t), A u^{(n)}(t)\right)\right| \lesssim\left\|u^{(n)}(t)\right\|_{V}^{1 / 2}\left\|A u^{(n)}(t)\right\|^{3 / 2} \lesssim \frac{\nu}{4}\left\|A u^{(n)}(t)\right\|^{2}+\left\|u^{(n)}(t)\right\|_{V}^{2} .
\end{aligned}
$$

Combining (3.19)-(3.22) gives

$$
\frac{\mathrm{d}}{\mathrm{d} t}\left\|u^{(n)}\right\|_{V}^{2}+\nu\left\|A u^{(n)}(t)\right\|^{2} \lesssim\|f(t)\|^{2}+2 L_{g}^{2}(t) e^{2 \gamma \rho(t)}\left\|u_{t}^{(n)}\right\|_{\gamma}^{2}+\left\|u^{(n)}(t)\right\|_{V}^{2} .
$$

Integrating (3.23) over $[s, t]$ with $t_{0}-T \leqslant s \leqslant t \leqslant t_{0}$ yields

$$
\left\|u^{(n)}(t)\right\|_{V}^{2} \lesssim\left\|u^{(n)}(s)\right\|_{V}^{2}+\int_{t_{0}-T}^{t_{0}}\left[\left(\|f(\theta)\|^{2}+2 L_{g}^{2}(\theta) e^{2 \gamma \rho(\theta)}\left\|u_{\theta}^{(n)}\right\|_{\gamma}^{2}\right)+\left\|u^{(n)}(\theta)\right\|_{V}^{2}\right] \mathrm{d} \theta .
$$

Now integrating (3.24) with respect to $s$ over $\left[t_{0}-T, t\right]$, we obtain for $t \in\left[t_{0}-\bar{T}, t_{0}\right]$ that

$$
\begin{aligned}
& (T-\bar{T})\left\|u^{(n)}(t)\right\|_{V}^{2} \leqslant\left(t+T-t_{0}\right)\left\|u^{(n)}(t)\right\|_{V}^{2} \\
\lesssim & T \int_{t_{0}-T}^{t_{0}}\left\|u^{(n)}(s)\right\|_{V}^{2} \mathrm{~d} s+T \int_{t_{0}-T}^{t_{0}}\left(\|f(s)\|^{2}+L_{g}^{2}(s) e^{2 \gamma \rho(s)}\left\|u_{s}^{(n)}\right\|_{\gamma}^{2}\right) \mathrm{d} s .
\end{aligned}
$$

In addition, from (3.8) and (3.14), we can get

$$
\begin{aligned}
\int_{\tau}^{t}\left\|u^{(n)}(s)\right\|_{V}^{2} \mathrm{~d} s \lesssim & \left(1+\int_{\tau}^{t} L_{g}(s) e^{\gamma \rho(s)} e^{2 \int_{\tau}^{s} L_{g}(\sigma) e^{\gamma \rho(\sigma)} \mathrm{d} \sigma} \mathrm{d} s\right)\|\phi\|_{\gamma}^{2} \\
& +\int_{\tau}^{t} e^{-2(\nu-\mu) \lambda_{1}(\tau-s)}\|f(s)\|^{2} \mathrm{~d} s \\
& +\int_{\tau}^{t} L_{g}(s) e^{\gamma \rho(s)} \int_{\tau}^{s} e^{-2(\nu-\mu) \lambda_{1}(\tau-\sigma)+2 \int_{\sigma}^{s} L_{g}(\theta) e^{\gamma \rho(\theta)} \mathrm{d} \theta}\|f(\sigma)\|^{2} \mathrm{~d} \sigma \mathrm{d} s .
\end{aligned}
$$


Note that we have $u^{(n)}\left(t ; \tau^{(n)}, \phi_{(n)}\right)=U\left(t, t_{0}-T\right) u\left(t_{0}-T ; \tau_{n}, \phi_{(n)}\right)$. Thus from (3.8)-(3.9), (3.13) and (3.15), we know that

$$
\text { the sequence }\left\{u^{(n)}\right\}_{n \geqslant n_{0}} \text { is bounded in } L^{\infty}\left(t_{0}-\bar{T}, t_{0} ; V\right) \text {. }
$$

Therefore, it follows from the compact embedding $V \hookrightarrow H$ and the continuity of $u^{(n)}$ (see (2.10)) that the set

$$
\left\{u^{n}(t): t \in\left[t_{0}-\bar{T}, t_{0}\right], n \geqslant n_{0}\right\} \text { is precompact in } H,
$$

from which (3.18) immediately follows.

Step two. We establish the equicontinuity of $\left\{u^{(n)}\right\}_{n \geqslant n_{0}}$ on $\left[t_{0}-\bar{T}, t_{0}\right]$ by contradiction.

Suppose that this equicontinuity does not hold true, and then there exists a positive constant $\varepsilon_{0}$ and two sequences $\left\{t_{n}^{(1)}\right\}$ and $\left\{t_{n}^{(2)}\right\}$, satisfying $t_{0}-\bar{T} \leqslant t_{n}^{(1)} \leqslant t_{n}^{(2)} \leqslant t_{0}$ and $\left|t_{n}^{(2)}-t_{n}^{(1)}\right| \leqslant \frac{1}{n}$, such that

$$
\left\|u^{(n)}\left(t_{n}^{(1)}\right)-u^{(n)}\left(t_{n}^{(2)}\right)\right\| \geqslant \varepsilon_{0} .
$$

By (3.28), we can assume that $t_{n}^{(1)} \rightarrow t^{*}, u^{(n)}\left(t^{*}\right) \rightarrow z^{*}$ and $u^{(n)}\left(t_{n}^{(i)}\right) \rightarrow z^{(i)}(i=1,2)$ in $H$ as $n \rightarrow+\infty$. Evidently, $t_{n}^{(2)} \rightarrow t^{*}$ as $n \rightarrow+\infty$ and $t^{*} \in\left[t_{0}-\bar{T}, t_{0}\right]$. By (3.23), we know that

$$
\left\|z^{(1)}-z^{(2)}\right\| \geqslant \varepsilon_{0} .
$$

Set $y^{(n)}(t)=u^{(n)}(t)-u^{(n)}\left(t^{*}\right)$ for $n \geqslant n_{0}$. Then we obtain

$$
\begin{aligned}
& \frac{1}{2} \frac{\mathrm{d}}{\mathrm{d} t}\left\|y^{(n)}(t)\right\|^{2}+\nu\left\|y^{(n)}(t)\right\|_{V}^{2}+\nu \int_{\Omega} \nabla u^{(n)}\left(t^{*}\right) \cdot \nabla y^{(n)}(t) \mathrm{d} x+b_{N}\left(u^{(n)}(t), u^{(n)}(t), y^{(n)}(t)\right) \\
= & \left(f(t), y^{(n)}(t)\right)+\left(g\left(t, u^{(n)}(t-\rho(t))\right), y^{(n)}(t)\right), \quad t \in\left[t_{0}-\bar{T}, t_{0}\right] .
\end{aligned}
$$

Applying Schwartz's inequality and (g3), we have

$$
\begin{aligned}
\left|\int_{\Omega} \nabla u^{(n)}\left(t^{*}\right) \cdot \nabla y^{(n)}(t) \mathrm{d} x\right| & \leqslant \frac{1}{2}\left\|u^{(n)}\left(t^{*}\right)\right\|_{V}^{2}+\frac{1}{2}\left\|y^{(n)}(t)\right\|_{V}^{2}, \\
2\left|\left(f(t), y^{(n)}(t)\right)\right| & \leqslant\|f(t)\|^{2}+\left\|y^{(n)}(t)\right\|^{2}, \\
2\left|\left(g\left(t, u^{(n)}(t-\rho(t))\right), y^{(n)}(t)\right)\right| & \leqslant L_{g}^{2}(t) e^{2 \gamma \rho(t)}\left\|u_{t}^{(n)}\right\|_{\gamma}^{2}+\left\|y^{(n)}(t)\right\|^{2} .
\end{aligned}
$$

At the same time, by (2.3)-(2.6) and (2.7), we see that

$$
\begin{aligned}
\left.\mid b_{N}\left(u^{(n)}(t), u^{(n)}(t)\right), y^{(n)}(t)\right) \mid & =\left|b_{N}\left(y^{(n)}(t), u^{(n)}(t), y^{(n)}(t)\right)-b_{N}\left(u^{(n)}\left(t^{*}\right), u^{(n)}(t), u^{(n)}\left(t^{*}\right)\right)\right| \\
& \lesssim\left\|y^{(n)}(t)\right\|_{V}^{3 / 2}\left\|y^{(n)}(t)\right\|^{1 / 2}+\left\|u^{(n)}\left(t^{*}\right)\right\|_{V}^{2} \\
& \lesssim \frac{\nu}{2}\left\|y^{(n)}(t)\right\|_{V}^{2}+\left\|y^{(n)}(t)\right\|^{2}+\left\|u^{(n)}\left(t^{*}\right)\right\|_{V}^{2}
\end{aligned}
$$

Taking (3.31)-(3.35) into account yields

$$
\frac{\mathrm{d}}{\mathrm{d} t}\left\|y^{(n)}(t)\right\|^{2} \lesssim\left\|y^{(n)}(t)\right\|^{2}+\|f(t)\|^{2}+L_{g}^{2}(t) e^{2 \gamma \rho(t)}\left\|u_{t}^{(n)}\right\|_{\gamma}^{2}+\left\|u^{(n)}\left(t^{*}\right)\right\|_{V}^{2} .
$$


Integrating (3.36) from $t^{*}$ to $t_{n}^{(i)}$ and using (3.16)- (3.17) gives

$$
\begin{aligned}
\left\|y^{(n)}\left(t_{n}^{i}\right)\right\|^{2} \leqslant & {\left[R\left(t_{0}, T\right)+c_{1}\right]\left|t_{n}^{i}-t^{*}\right|+\left|\int_{t^{*}}^{t_{n}^{i}}\|f(s)\|^{2} \mathrm{~d} s\right| } \\
& +R\left(t_{0}, T\right)\left|\int_{t^{*}}^{t_{n}^{i}} L_{g}^{2}(s) e^{2 \gamma \rho(s)} \mathrm{d} s\right|
\end{aligned}
$$

where (see (2.10) and (3.27))

$$
c_{1}:=\sup _{t \in\left[t_{0}-\bar{T}, t_{0}\right]}\left\{\left\|u_{n}(t)\right\|_{V}: n \geqslant n_{0}\right\}
$$

Letting $n \rightarrow+\infty$ in (3.37) and using the assumptions $f \in L_{\mathrm{loc}}^{2}\left(\mathbb{R} ; \mathbb{L}^{2}(\Omega)\right)$ and (g3), we conclude that

$$
\left\|z^{i}-z^{*}\right\|^{2}=\lim _{n \rightarrow+\infty}\left\|u^{(n)}\left(t_{n}^{i}\right)-u^{(n)}\left(t^{*}\right)\right\|^{2}=0, \quad \text { for } i=1,2,
$$

which contradicts (3.30).

At this stage, we can apply the Ascoli-Arzelà type theorem [1] to claim that for each $\bar{T}>0$, $\left\{u^{(n)}\right\}_{n \in \mathbb{N}_{+}}$is precompact in $C\left(\left[t_{0}-\bar{T}, t_{0}\right] ; H\right)$. Thus there exists a function $\psi \in C([-\bar{T}, 0] ; H)$ and a subsequence of $u_{t_{0}}^{(n)}$ such that $\left.u_{t_{0}}^{(n)}\right|_{[-\bar{T}, 0]} \rightarrow \psi$ in $C([-\bar{T}, 0] ; H)$. Repeating this procedure for $n \bar{T}$ with $n=2,3, \cdots$, and using the diagonal procedure, we can obtain a function $\psi \in C((-\infty, 0] ; H)$ such that (extract a subsequence if necessary) $\left.u_{t_{0}}^{(n)}\right|_{[-\bar{T}, 0]} \rightarrow \psi$ in $C([-\bar{T}, 0] ; H)$ for each positive number $\bar{T}$. The proof of Lemma 3.2 is complete.

Lemma 3.3. Let the assumptions (H1) and (H2) hold. Then the process $\{U(t, \tau)\}_{t \geqslant \tau}$ is pullback $\mathcal{D}_{\sigma^{-}}$ asymptotically compact in $C_{\gamma}(H)$.

Proof. We continue to use the notations of the previous lemma. To establish the pullback $\mathcal{D}_{\sigma}$-asymptotic compactness, we shall prove that $u_{t_{0}}^{n} \rightarrow \psi$ in $C_{\gamma}(H)$, for which, it is sufficient to show that, for every $\varepsilon>0$, there exists some positive integer $n_{\varepsilon}$ such that

$$
\sup _{s \in(-\infty, 0]} e^{2 \gamma s}\left\|u_{t_{0}}^{(n)}(s)-\psi(s)\right\|^{2}<\varepsilon, \quad \text { for all } n \geqslant n_{\varepsilon} .
$$

Indeed, by (3.16)-(3.17) and the convergence proved in the previous lemma, we have that

$$
\|\psi(s)\|^{2} \lesssim 1+e^{-\sigma\left(t_{0}-T\right)} \int_{-\infty}^{t_{0}} e^{\sigma s}\|f(s)\|^{2} \mathrm{~d} s=R\left(t_{0}, T\right), \quad \text { for all } s \in[-T, 0] \text { and } T>0 .
$$

The rest proof is similar to that of [31, Proposition 3] and we omit the details here.

At this stage we combine Lemmas 3.1, 3.3 and the general result of [19, Theorem 3.11] to obtain the main result of this section, namely,

Theorem 3.1. Let the assumptions $(\mathbf{H 1})$ and $(\mathbf{H 2})$ hold. Then the process $\{U(t, \tau)\}_{t \geqslant \tau}$ possesses a pullback $\mathcal{D}_{\sigma}$-attractor (denoted by) $\hat{\mathcal{A}}_{\mathcal{D}_{\sigma}}^{H}=\left\{\mathcal{A}_{\mathcal{D}_{\sigma}}^{H}(t): t \in \mathbb{R}\right\}$ in $C_{\gamma}(H)$. 


\section{Existence of the invariant Borel probability measures in $C_{\gamma}(H)$}

In this section, we first aim to prove that for each given $t \in \mathbb{R}$ and $\phi \in C_{\gamma}(H)$, the $C_{\gamma}(H)$-valued function $\tau \mapsto U(t, \tau) \phi$ is bounded and continuous on $(-\infty, t]$. Then we combine Theorem 3.1 and the abstract result of [28, Theorem 3.1] to obtain the existence of invariant Borel probability measures for $\{U(t, \tau)\}_{t \geqslant \tau}$ in $C_{\gamma}(H)$.

Lemma 4.1. Let the assumptions (H1) and (H2) hold. Then for every $\phi \in C_{\gamma}(H)$ and every $t \in \mathbb{R}$ the $C_{\gamma}(H)$-valued function $\tau \mapsto U(t, \tau) \phi$ is bounded on $(-\infty, t]$.

Proof. Let $\phi \in C_{\gamma}(H)$ and $t \in \mathbb{R}$ be given. Note (3.4) is equivalent to (see [31, Remark 5])

$$
\int_{-\infty}^{t} e^{\sigma s}\|f(s)\|^{2} \mathrm{~d} s<+\infty \quad \text { for all } t \in \mathbb{R} .
$$

Then (3.14) and (4.1) imply that for all $\tau \in(-\infty, t]$,

$$
\|U(t, \tau) \phi\|_{\gamma}^{2} \lesssim e^{-\sigma(t-\tau)}\|\phi\|_{\gamma}^{2}+\int_{\tau}^{t} e^{-\sigma(t-s)}\|f(s)\|^{2} \mathrm{~d} s \leqslant\|\phi\|_{\gamma}^{2}+e^{-\sigma t} \int_{-\infty}^{t} e^{\sigma s}\|f(s)\|^{2} \mathrm{~d} s
$$

the right-hand side of which is bounded by a positive constant independent of $\tau \in(-\infty, t]$.

In the sequel, we set a positive number $M(t, \phi)$ such that

$$
M^{2}(t, \phi):=\|\phi\|_{\gamma}^{2}+e^{-\sigma t} \int_{-\infty}^{t} e^{\sigma s}\|f(s)\|^{2} \mathrm{~d} s .
$$

Then $M^{2}(t, \phi)$ is a continuous function with respect to $t \in \mathbb{R}$.

To establish the continuity of the $C_{\gamma}(H)$-valued function $\tau \mapsto U(t, \tau) \phi$ on $(-\infty, t]$, we next prove two auxiliary lemmas.

Lemma 4.2. Let $\phi \in C_{\gamma}(H)$ be given. Then for each $\varepsilon>0$, there exists some $\delta_{1}=\delta_{1}(\varepsilon, \phi)>0$ such that for all $s_{1}, s_{2} \in(-\infty, 0]$ with $\left|s_{1}-s_{2}\right|<\delta_{1}$ there holds

$$
e^{\gamma s_{2}}\left\|\phi\left(s_{1}\right)-\phi\left(s_{2}\right)\right\|<\varepsilon
$$

Proof. By the definition of $C_{\gamma}(H)$, we set $\phi_{\infty}:=\lim _{s \rightarrow-\infty} e^{\gamma s} \phi(s) \in H$. Then there exists some $s_{0}<0$ such that

$$
\left\|e^{\gamma s} \phi(s)-\phi_{\infty}\right\|<\frac{\varepsilon}{4}, \forall s \leqslant s_{0}
$$

and thus

$$
\left\|e^{\gamma s_{1}} \phi\left(s_{1}\right)-e^{\gamma s_{2}} \phi\left(s_{2}\right)\right\| \leqslant\left\|e^{\gamma s_{1}} \phi\left(s_{1}\right)-\phi_{\infty}\right\|+\left\|e^{\gamma s_{2}} \phi\left(s_{2}\right)-\phi_{\infty}\right\|<\frac{\varepsilon}{2}, \forall s_{1}, s_{2} \leqslant s_{0} .
$$

On the other hand, the $H$-valued function $s \mapsto e^{\gamma s} \phi(s)$ is uniformly continuous on the interval $\left[s_{0}-1,0\right]$, whence there is some $\delta_{1}^{\prime} \in(0,1)$ such that

$$
\left\|e^{\gamma s_{1}} \phi\left(s_{1}\right)-e^{\gamma s_{2}} \phi\left(s_{2}\right)\right\|<\frac{\varepsilon}{2} \quad \text { for } s_{1}, s_{2} \in\left[s_{0}-1,0\right] \text { with }\left|s_{1}-s_{2}\right|<\delta_{1}^{\prime} .
$$


Letting $\delta_{1}=\min \left\{\delta_{1}^{\prime}, \frac{1}{\gamma} \ln \left(1+\frac{\varepsilon}{2\|\phi\|_{\gamma}}\right)\right\}$, we have by (4.3) and (4.4) that for all $s_{1}, s_{2} \in(-\infty, 0]$ with $\left|s_{1}-s_{2}\right|<\delta_{1}$ there holds

$$
\begin{aligned}
& e^{\gamma s_{2}}\left\|\phi\left(s_{1}\right)-\phi\left(s_{2}\right)\right\| \leqslant\left\|e^{\gamma s_{1}} \phi\left(s_{1}\right)-e^{\gamma s_{2}} \phi\left(s_{2}\right)\right\|+\left|e^{\gamma s_{1}}-e^{\gamma s_{2}}\right|\left\|\phi\left(s_{1}\right)\right\| \\
\leqslant & \frac{\varepsilon}{2}+\left|e^{\gamma\left(s_{2}-s_{1}\right)}-1\right|\|\phi\|_{\gamma}<\varepsilon .
\end{aligned}
$$

The proof is complete.

Lemma 4.3. Let the assumptions (H1) and (H2) hold, and $\tau_{*} \in \mathbb{R}$ and $\phi \in C_{\gamma}(H)$ be given. Then for each $\varepsilon>0$, there exists some $\delta_{2}=\delta_{2}\left(\varepsilon, \tau_{*}, \phi\right)>0$ such that

$$
\|u(s ; \tau, \phi)-\phi(0)\|<\varepsilon, \quad \text { for all } \tau \in\left(\tau_{*}-\delta_{2}, \tau_{*}\right) \text { and } s \in\left[\tau, \tau_{*}\right],
$$

where $u(s ; \tau, \phi)$ is the solution of the problem (1.1) with the initial datum $\phi$ at the initial time $\tau$.

Proof. Let $\tau_{*} \in \mathbb{R}$ and $\phi \in C_{\gamma}(H)$ be given. Firstly, we prove that

$$
\int_{\tau}^{\tau_{*}}\left\|\frac{\mathrm{d} u(\theta ; \tau, \phi)}{\mathrm{d} \theta}\right\|_{V^{\prime}}^{2} \mathrm{~d} \theta \leqslant c_{2}
$$

where

$$
c_{2}:=\|\phi\|_{\gamma}^{2}+\int_{\tau_{*}-1}^{\tau_{*}}\|f(\theta)\|^{2} \mathrm{~d} \theta+\sup _{\left[\tau_{*}-1, \tau_{*}\right]} M^{2}(\theta, \phi),
$$

is a bounded quantity depending on $\tau_{*}, g, f, \cdots$, etc., but being independent of $\tau \in\left[\tau_{*}-1, \tau_{*}\right]$. Indeed, from (2.9) we can see that

$$
\begin{gathered}
\left\|\frac{\mathrm{d} u(\theta ; \tau, \phi)}{\mathrm{d} \theta}\right\|_{V^{\prime}}^{2} \lesssim \\
+\|A u(\theta ; \tau, \phi)\|_{V^{\prime}}^{2}+\left\|B_{N}(u(\theta ; \tau, \phi), u(\theta ; \tau, \phi))\right\|_{V^{\prime}}^{2} \\
+\|f(\theta)\|^{2}+\|g(\theta, u(\theta-\rho(\theta)))\|^{2} .
\end{gathered}
$$

By (2.5), (g2), (g3) and some simple observations, we have

$$
\left\{\begin{array}{l}
\|A u(\theta ; \tau, \phi)\|_{V^{\prime}}^{2} \lesssim\|u(\theta ; \tau, \phi)\|_{V}^{2}, \\
\left\|B_{N}(u(\theta ; \tau, \phi), u(\theta ; \tau, \phi))\right\|_{V^{\prime}}^{2} \lesssim\|u(\theta ; \tau, \phi)\|_{V}^{2} \\
\|g(\theta, u(\theta-\rho(\theta)))\|^{2} \lesssim \sup _{\left[\tau_{*}-1, \tau_{*}\right]} M^{2}(\theta, \phi) .
\end{array}\right.
$$

Inserting (4.7) and (4.8) into (4.6), then using (3.3), (3.5) and (3.26) gives

$$
\begin{aligned}
\int_{\tau}^{\tau_{*}}\left\|\frac{\mathrm{d} u(\theta ; \tau, \phi)}{\mathrm{d} \theta}\right\|_{V^{\prime}}^{2} \mathrm{~d} \theta & \left.\lesssim \int_{\tau}^{\tau_{*}} \| u(\theta ; \tau, \phi)\right)\left\|_{V}^{2} \mathrm{~d} \theta+\int_{\tau}^{\tau_{*}}\right\| f(\theta) \|^{2} \mathrm{~d} \theta+\left(\tau_{*}-\tau\right) \sup _{\left[\tau_{*}-1, \tau_{*}\right]} M^{2}(\theta, \phi) \\
& \lesssim\|\phi\|_{\gamma}^{2}+\int_{\tau_{*}-1}^{\tau_{*}}\|f(\theta)\|^{2} \mathrm{~d} \theta+\sup _{\left[\tau_{*}-1, \tau_{*}\right]} M^{2}(\theta, \phi), \forall \tau \in\left[\tau_{*}-1, \tau_{*}\right]
\end{aligned}
$$

that is (4.6) is proved. 
Secondly, we observe that for $\tau_{*}-1 \leqslant \tau<s \leqslant \tau_{*}$ there holds

$$
\begin{aligned}
& \|u(s ; \tau, \phi)-\phi(0)\|^{2} \\
= & \|u(s ; \tau, \phi)\|^{2}-\|\phi(0)\|^{2}-2(u(s ; \tau, \phi)-\phi(0), \phi(0)) \\
= & \int_{\tau}^{s} \frac{\mathrm{d}\|u(\theta ; \tau, \phi)\|^{2}}{\mathrm{~d} \theta} \mathrm{d} \theta-2(u(s ; \tau, \phi)-\phi(0), \phi(0)) .
\end{aligned}
$$

By (3.3) and (3.13), we obtain

$$
\begin{aligned}
\left|\int_{\tau}^{s} \frac{\mathrm{d}\|u(\theta ; \tau, \phi)\|^{2}}{\mathrm{~d} \theta} \mathrm{d} \theta\right| & \lesssim \int_{\tau}^{s}\|f(\theta)\|^{2} \mathrm{~d} \theta+\int_{\tau}^{s} L_{g}(\theta) e^{\gamma \rho(\theta)}\left\|u_{\theta}\right\|_{\gamma}^{2} \mathrm{~d} \theta \\
& \lesssim \int_{\tau}^{\tau_{*}}\|f(\theta)\|^{2} \mathrm{~d} \theta+\left(\tau_{*}-\tau\right) \sup _{\theta \in\left[\tau_{*}-1, \tau_{*}\right]} M^{2}(\theta, \phi) .
\end{aligned}
$$

Notice that $f \in L_{\mathrm{loc}}^{2}\left(\mathbb{R}, \mathbb{L}^{2}(\Omega)\right)$, and $\sup _{\theta \in\left[\tau_{*}-1, \tau_{*}\right]} M^{2}(\theta, \phi)$ is a constant independent of $\tau$. Therefore, from (4.11) we see that there exists some $\delta_{2}^{\prime}=\delta_{2}^{\prime}\left(\varepsilon, \tau_{*}, \phi\right) \in(0,1)$ such that

$$
\left|\int_{\tau}^{s} \frac{\mathrm{d}\|u(\theta ; \tau, \phi)\|^{2}}{\mathrm{~d} \theta} \mathrm{d} \theta\right|<\frac{\varepsilon^{2}}{2}, \quad \tau_{*}-\delta_{2}^{\prime}<\tau<s \leqslant \tau_{*} .
$$

At the same time, since $\phi(0) \in H$ and $V$ is dense in $H$, there exists some $\psi \in V$ such that

$$
\|\psi-\phi(0)\|<\varepsilon^{2} / 16 \sup _{\theta \in\left[\tau_{*}-1, \tau_{*}\right]} M^{2}(\theta, \phi) .
$$

Using (4.6),

$$
\begin{aligned}
|(u(s ; \tau, \phi)-\phi(0), \phi(0))| & \leqslant|(u(s ; \tau, \phi)-\phi(0), \phi(0)-\psi)|+|(u(s ; \tau, \phi)-\phi(0), \psi)| \\
& \leqslant 2 \sup _{\theta \in\left[\tau_{*}-1, \tau_{*}\right]} M(\tau, \phi)\|\phi(0)-\psi\|+|\langle u(s ; \tau, \phi)-\phi(0), \psi\rangle| \\
& <\frac{\varepsilon^{2}}{8}+\left|\left\langle\int_{\tau}^{s} \frac{\mathrm{d} u(\theta ; \tau, \phi)}{\mathrm{d} \theta} \mathrm{d} \theta, \psi\right\rangle\right| \\
& \leqslant \frac{\varepsilon^{2}}{8}+\|\psi\|_{V} \sqrt{\tau_{*}-\tau}\left(\int_{\tau}^{\tau_{*}}\left\|\frac{\mathrm{d} u(\theta ; \tau, \phi)}{\mathrm{d} \theta}\right\|_{V^{\prime}}^{2} \mathrm{~d} \theta\right)^{\frac{1}{2}} \\
& \leqslant \frac{\varepsilon^{2}}{8}+\sqrt{c_{2}}\|\psi\|_{V} \sqrt{\tau_{*}-\tau}
\end{aligned}
$$

which implies that there exists some $\delta_{2}^{\prime \prime} \in(0,1)$, independent of $\tau$, such that

$$
|(u(s ; \tau, \phi)-\phi(0), \phi(0))| \leqslant \frac{\varepsilon^{2}}{4}, \quad \tau_{*}-\delta_{2}^{\prime \prime}<\tau<s \leqslant \tau_{*} .
$$

Picking $\delta_{2}=\min \left\{\delta_{2}^{\prime}, \delta_{2}^{\prime \prime}\right\}$, the desired result follows from (4.10), (4.12) and (4.14). The proof of Lemma 4.3 is complete.

Lemma 4.4. Let the assumptions (H1) and (H2) hold. Then for every $\phi \in C_{\gamma}(H)$ and every $t \in \mathbb{R}$ the $C_{\gamma}(H)$-valued function $\tau \mapsto U(t, \tau) \phi$ is continuous on $(-\infty, t]$. 
Proof. Let $\phi \in C_{\gamma}(H)$ and $t \in \mathbb{R}$ be given. We shall prove that for each given $\tau_{*} \in(-\infty, t]$ the $C_{\gamma}(H)$-valued function $\tau \mapsto U(t, \tau) \phi$ is both left and right continuous at $\tau=\tau_{*}$. We next prove the left continuity at $\tau=\tau_{*}$.

Firstly, on one hand, for every $\varepsilon>0$ we deduce from Lemma 4.2 that there exists some $\delta_{3}^{\prime}=$ $\delta_{3}^{\prime}(\varepsilon, \phi)>0$ such that, for all $s_{1}, s_{2} \in(-\infty, 0]$ with $\left|s_{1}-s_{2}\right|<\delta_{1}$, there holds

$$
e^{\gamma s_{2}}\left\|\phi\left(s_{1}\right)-\phi\left(s_{2}\right)\right\|<\varepsilon / 2 \text {. }
$$

On the other hand, by Lemma 4.3 , there is a $\delta_{3}^{\prime \prime}=\delta_{3}^{\prime \prime}\left(\varepsilon, \tau_{*}, \phi\right)>0$ such that whenever $\tau \in\left(\tau_{*}-\delta_{3}^{\prime \prime}, \tau_{*}\right)$ there holds

$$
\|u(s ; \tau, \phi)-\phi(0)\|<\varepsilon / 2, \quad \text { for all } s \in\left[\tau, \tau_{*}\right] .
$$

Thus, for the positive $\varepsilon$ above, there exists some $\delta_{3}=\delta_{3}\left(\varepsilon, \tau_{*}, \phi\right)=\min \left\{\delta_{3}^{\prime}, \delta_{3}^{\prime \prime}\right\}>0$ such that

$$
\begin{aligned}
\left\|u_{*}(\theta)-u(\theta)\right\| & =\|\phi(\theta-\tau)-u(\theta)\| \\
& \leqslant\|\phi(\theta-\tau)-\phi(0)\|+\|\phi(0)-u(\theta ; \tau, \phi)\|<\varepsilon, \quad \tau_{*}-\delta_{3}<\tau<\tau_{*}, \quad \theta \in\left[\tau, \tau_{*}\right],
\end{aligned}
$$

which implies that

$$
\max _{\theta \in\left[\tau, \tau_{*}\right]}\left\|u_{*}\left(\theta ; \tau_{*}, \phi\right)-u(\theta ; \tau, \phi)\right\|<\varepsilon, \quad \tau_{*}-\delta_{3}<\tau<\tau_{*}, \quad \theta \in\left[\tau, \tau_{*}\right]
$$

where $u_{*}\left(\theta ; \tau_{*}, \phi\right)$ is the solution corresponding to the initial datum $\phi$ at the initial time $\tau_{*}$.

Secondly, by (2.18) and (3.3) we have

$$
\begin{aligned}
\max _{\theta \in\left[\tau_{*}, t\right]} & \left\|u_{*}\left(\theta ; \tau_{*}, \phi\right)-u(\theta ; \tau, \phi)\right\| \\
\lesssim & \left\|\phi(0)-u\left(\tau_{*} ; \tau, \phi\right)\right\|^{2} \exp \left(\int_{\tau_{*}}^{t} L_{g}(s) e^{\gamma \rho(s)} \mathrm{d} s+\left(t-\tau_{*}\right)\right) \\
& \quad+\left\|\phi-u_{\tau_{*}}\right\|_{\gamma}^{2} \int_{\tau_{*}}^{t} L_{g}(s) e^{\gamma \rho(s)} \exp \left(\int_{\tau_{*}}^{s} L_{g}(\theta) e^{\gamma \rho(\theta)} \mathrm{d} \theta+\left(s-\tau_{*}\right)\right) \mathrm{d} s . \\
& \lesssim\left\|\phi(0)-u\left(\tau_{*} ; \tau, \phi\right)\right\|^{2}+\left\|\phi-u_{\tau_{*}}\right\|_{\gamma}^{2},
\end{aligned}
$$

using the representation $u(\theta ; \tau, \phi)=u\left(\theta ; \tau_{*}, u\left(\tau_{*} ; \tau, \phi\right)\right)$ and the fact that $t$ and $\tau_{*}$ are fixed. Now by Lemma 4.3, we derive that for above $\varepsilon>0$ there exists $\delta_{4}^{\prime}=\delta_{4}^{\prime}\left(\varepsilon, t, \tau_{*}, \phi\right)>0$ such that

$$
\left\|\phi(0)-u\left(\tau_{*} ; \tau, \phi\right)\right\|<\varepsilon / 2, \text { for } \tau_{*}-\delta_{4}^{\prime}<\tau<\tau_{*} .
$$

At the same time, by (4.15) and (4.17), there exists $\delta_{4}^{\prime \prime}=\delta_{4}^{\prime \prime}\left(\varepsilon, \tau_{*}, \phi\right)>0$ such that when $\tau_{*}-\delta_{4}^{\prime \prime}<\tau<$ $\tau_{*}$ then

$$
\begin{aligned}
\left\|\phi-u_{\tau_{*}}\right\|_{\gamma} & \leqslant \max \left\{\sup _{s \leqslant \tau_{*}-\tau} e^{\gamma s}\left\|\phi(s)-u_{\tau_{*}}(s)\right\|, \sup _{s \in\left[\tau-\tau_{*}, 0\right]} e^{\gamma s}\left\|\phi(s)-u_{\tau_{*}}(s)\right\|\right\} \\
& \leqslant \max \left\{\sup _{s \leqslant \tau-\tau_{*}} e^{\gamma s}\left\|\phi(s)-\phi\left(s+\tau_{*}-\tau\right)\right\|, \sup _{s \in\left[\tau, \tau_{*}\right]}\left\|u_{*}(s)-u(s)\right\|\right\} \\
& \leqslant \max \left\{\sup _{r \leqslant 0} e^{\gamma\left(r+\tau-\tau_{*}\right)}\left\|\phi\left(r+\tau-\tau_{*}\right)-\phi(r)\right\|, \varepsilon\right\}<\varepsilon / 2 .
\end{aligned}
$$


Picking $\delta_{4}=\delta_{4}\left(\varepsilon, t, \tau_{*}, \phi\right)=\min \left\{\delta_{4}^{\prime}, \delta_{4}^{\prime \prime}\right\}$, then (4.18)-(4.20) imply

$$
\max _{\theta \in\left[\tau_{*}, t\right]}\left\|u_{*}\left(\theta ; \tau_{*}, \phi\right)-u(\theta ; \tau, \phi)\right\|<\varepsilon, \quad \tau_{*}-\delta_{4}<\tau<\tau_{*}<\varepsilon .
$$

We now choose $\delta=\delta\left(\varepsilon, t, \tau_{*}, \phi\right)=\min \left\{\delta_{3}, \delta_{4}\right\}$, and then deduce from (4.15), (4.17) and (4.21) that if $\tau_{*}-\delta<\tau<\tau_{*}$ then

$$
\begin{aligned}
& \left\|U(t, \tau) \phi-U\left(t, \tau_{*}\right) \phi\right\|_{\gamma}=\sup _{\theta \leqslant 0} e^{\gamma \theta}\left\|u(t+\theta)-u_{*}(t+\theta)\right\|_{\gamma} \\
\leqslant & \max \left\{\sup _{\theta \leqslant \tau-t} e^{\gamma \theta}\left\|\phi\left(\theta+t-\tau_{*}\right)-\phi(\theta+t-\tau)\right\|, \sup _{\theta \in[\tau-t, 0]} e^{\gamma \theta}\left\|u(t+\theta)-u_{*}(t+\theta)\right\|\right\} \\
\leqslant & \max \left\{\sup _{r \leqslant 0} e^{\gamma\left(r+\tau_{*}-t\right)}\left\|\phi(r)-\phi\left(r+\tau_{*}-\tau\right)\right\|, \sup _{\theta \in[\tau, t]}\left\|u(\theta)-u_{*}(\theta)\right\|\right\}<\varepsilon .
\end{aligned}
$$

The left continuity of the $C_{\gamma}(H)$-valued function $\tau \mapsto U(t, \tau) \phi$ at $\tau=\tau_{*}$ is proved. Since the proof of the right continuity of $U(t, \tau) \phi$ at $\tau=\tau_{*}$ is similar to that of the left continuity, we omit the details and end the proof of Lemma 4.4.

To construct the invariant measures for the process $\{U(t, \tau)\}_{t \geqslant \tau}$, we next recall the definition of generalized Banach limits.

Definition 4.1. ( $[16,28])$ A generalized Banach limit is any linear functional, denoted by $\mathrm{LIM}_{t \rightarrow+\infty}$, defined on the space of all bounded real-valued functions on $[0,+\infty)$ and satisfying

(1) $\operatorname{LIM}_{t \rightarrow+\infty} \zeta(t) \geqslant 0$ for nonnegative functions $\zeta(\cdot)$ on $[0,+\infty)$;

(2) $\operatorname{LIM}_{t \rightarrow+\infty} \zeta(t)=\lim _{t \rightarrow+\infty} \zeta(t)$ if the usual limit $\lim _{t \rightarrow+\infty} \zeta(t)$ exists.

Remark 4.1. Notice that we consider the "pullback" asymptotic behavior and we require generalized limits as $\tau \rightarrow-\infty$. For a given real-valued function $\zeta$ defined on $(-\infty, 0]$ and a given Banach limit $\operatorname{LIM}_{t \rightarrow+\infty}$, we define $\operatorname{LIM}_{t \rightarrow-\infty} \zeta(t)=\operatorname{LIM}_{t \rightarrow+\infty} \zeta(-t)$.

The main result of this article reads as follows.

Theorem 4.1. Let the assumptions (H1) and (H2) hold. Let $\{U(t, \tau)\}_{t \geqslant \tau}$ be the process associated to the problem (1.1) and $\widehat{\mathcal{A}}_{\mathcal{D}_{\sigma}}=\left\{\mathcal{A}_{\mathcal{D}_{\sigma}}(t): t \in \mathbb{R}\right\}$ the pullback $\mathcal{D}_{\sigma}$-attractor obtained in Theorem 3.1. Then for a given generalized Banach limit $\operatorname{LIM}_{t \rightarrow+\infty}$ and a continuous map $\xi_{\tau}: \mathbb{R} \longmapsto C_{\gamma}(H)$ with $\xi_{\tau}(\cdot) \in \mathcal{D}_{\sigma}$, there exists a unique family of Borel probability measures $\left\{m_{t}\right\}_{t \in \mathbb{R}}$ in $C_{\gamma}(H)$ such that the support of the measure $m_{t}$ is contained in $\mathcal{A}_{\mathcal{D}_{\sigma}}(t)$ and

$$
\begin{aligned}
\operatorname{LIM}_{\tau \rightarrow-\infty} \frac{1}{t-\tau} \int_{\tau}^{t} \Upsilon\left(U(t, s) \xi_{s}\right) \mathrm{d} s & =\int_{\mathcal{A}_{\mathcal{D}_{\sigma}}(t)} \Upsilon(u) \mathrm{d} m_{t}(u) \\
& =\int_{C_{\gamma}(H)} \Upsilon(u) \mathrm{d} m_{t}(u) \\
& =\operatorname{LIM}_{\tau \rightarrow-\infty} \frac{1}{t-\tau} \int_{\tau}^{t} \int_{C_{\gamma}(H)} \Upsilon(U(t, s) u) \mathrm{d} m_{s}(u) \mathrm{d} s
\end{aligned}
$$


for every real-valued continuous functional $\Upsilon$ on $C_{\gamma}(H)$. Moreover, $m_{t}$ is invariant in the sense that

$$
\int_{\mathcal{A}_{\mathcal{D}_{\sigma}}(t)} \Upsilon(u) \mathrm{d} m_{t}(u)=\int_{\mathcal{A}_{\mathcal{D}_{\sigma}}(\tau)} \Upsilon(U(t, \tau) u) \mathrm{d} m_{\tau}(u), \quad t \geqslant \tau .
$$

Proof. From Theorems 2.1 and 2.2, the solution operators of the problem (1.1) generate a continuous process $\{U(t, \tau)\}_{t \geqslant \tau}$ on the space $C_{\gamma}(H)$. Theorem 3.1 shows that $\{U(t, \tau)\}_{t \geqslant \tau}$ possesses a pullback attractor in $C_{\gamma}(H)$. Lemmas 4.1 and 4.4 indicate that for each given $t \in \mathbb{R}$ and $\phi \in C_{\gamma}(H)$ the $C_{\gamma}(H)$ valued function $\tau \mapsto U(t, \tau) \phi$ is bounded and continuous on $(-\infty, t]$. Using these facts and the abstract result of [28, Theorem 3.1], we obtain the results of Theorem 4.1.

\section{Conclusions and remarks}

In this article, we first prove the global well-posedness of the 3D GMNS equations with unbounded variable delays. Then we establish that its solution operators generate a continuous process $\{U(t, \tau)\}_{t \geqslant \tau}$ on the space $C_{\gamma}(H)$. Furthermore, the process $\{U(t, \tau)\}_{t \geqslant \tau}$ possesses a pullback attractor in $C_{\gamma}(H)$. Finally, we prove the existence of invariant Borel probability measures which are supported by the pullback attractor. There are two points that we would like to point out.

Firstly, we apply the generalized Ascoli-Arzelà theorem to prove the "weak version" of the pullback asymptotic compactness of the process $\{U(t, \tau)\}_{t \geqslant \tau}$ (see Lemma 3.2). This is prompted by the delay phenomenon. Since the problem discussed contains unbounded variable delays, it seems suitable for us to choose $C_{\gamma}(H)$ as the phase space. The Ascoli-Arzelà theorem has been proved to be a powerful tool to investigate the compactness of the sets such as the subset of $C_{\gamma}(H)$.

Secondly, for each given $t \in \mathbb{R}$ and $\phi \in C_{\gamma}(H)$, the continuity of the $C_{\gamma}(H)$-valued function $\tau \mapsto$ $U(t, \tau) \phi$ on $(-\infty, t]$ is not a clear fact (see Lemma 4.4 and the auxiliary lemmas). There are some essential differences between the autonomous dynamical system and non-autonomous one, say the continuous dependence of the dynamical system on their parameters. Consider a continuous process $\{S(t, \tau)\}_{t \geqslant \tau}$ on some Banach space $X$. Even if we have known that the $X$-valued function $t \mapsto S(t, \tau) x_{0}$ is continuous on $\mathbb{R}$ for every given $\tau$ and $x_{0} \in X$, the convergence $\left\|S\left(t_{*}, \tau\right) x_{0}-x_{0}\right\|_{X} \rightarrow 0$ as $\tau \rightarrow t_{*}^{-}$may still depend on $\tau$. In fact, when $\tau \rightarrow t_{*}^{-}, S\left(t_{*}, \tau\right) x_{0}$ changes simultaneously with different initial times $\tau$. This is caused naturally by the non-autonomous phenomenon.

In the end of this article, we want to propose a problem, saying, how to formulate reasonably and construct the statistical solutions for the evolution system containing delays. As for the 3D GMNS equations discussed here, we have constructed the invariant measures on the phase space $C_{\gamma}(H)$. However, we seem unable to construct its statistical solutions via the invariant measures and pullback attractors, as done in [44-47], because the invariant measures are contained in the phase space $C_{\gamma}(H)$ while the equation (2.9) is interpreted in the $\mathcal{D}\left(\tau,+\infty ; V^{\prime}\right)$ sense.

\section{Acknowledgement}

This work was supported by NSF of China with No.11801190, 11971356 and by NSF of Zhejiang Province with No.LY17A010011, and also supported by FEDER and the Spanish Ministerio de Ciencia, Innovación y Universidades project PGC2018-096540-B-I00. 


\section{References}

[1] J. P. Aubin, A. Cellina, Differential Inclusions, Springer-Verlag, New York, 1984.

[2] T. Caraballo, J. Real, Navier-Stokes equations with delays, R. Soc. Lond. Proc. Ser. A Math. Phys. Eng. Sci., 457 (2001), 2441-2453.

[3] T. Caraballo, J. Real, Asymptotic behaviour of two-dimensional Navier-Stokes equations with delays, R. Soc. Lond. Proc. Ser. A Math. Phys. Eng. Sci., 459 (2003), 3181-3194.

[4] T. Caraballo, J. Real, Attractors for 2D Navier-Stokes models with delays, J. Differential Equations, 205 (2004), 270-296.

[5] T. Caraballo, J. Real, P. E. Kloeden, Unique strong solutions and $V$-attractors of a three dimensional system of globally modified Navier-Stokes equations, Adv. Nonlinear Study, 6 (2006), 411-436.

[6] T. Caraballo, J. Real, P. E. Kloeden, Addendum to the paper "Unique strong solutions and $V$ attractors of a 3-dimensional system of globally modified Navier-Stokes equations, Advanced Nonlinear Studies 6 (2006), 411-436. Adv. Nonlinear Study, 10 (2006), 245-247.

[7] T. Caraballo, P. Marín-Rubio, J. Valero, Attractors for differential equations with unbounded delays, J. Differential Equations, 239 (2007), 311-342.

[8] T. Caraballo, P. E. Kloeden, J. Real, Invariant measures and statistical solutions of the globally modified Navier-Stokes equations, Discrete Cont. Dyn. Syst.-B, 10 (2008), 761-781.

[9] T. Caraballo, J. Real, A. M. Márquez-Durán, Three-dimensional system of globally modified NavierStokes equations with delay, Inter. J. Bifur. Chaos, 20 (2010), 2869-2883.

[10] A. N. Carvalho, J. A. Langa, J. C. Robinson, Attractors of Infinite Dimensional Nonautonomous Dynamical Systems, Applied Mathematical Sciences Vol. 182, Springer, New York, 2013.

[11] S. G. Chefranov, A. S. Chefranov, The new analytical solution of the 3D Navier-Stokes equation for compressible medium clarifies the sixth Millennium Prize problem, Cardiometry, (2017), 18-33.

[12] S. G. Chefranov, A. S. Chefranov, The new exact solution of the compressible 3D Navier-Stokes equations, Comm. Nonl. Sci. Numer. Simul., 83 (2020), 105118.

[13] M. Chekroun, N. E. Glatt-Holtz, Invariant measures for dissipative dynamical systems: Abstract results and applications, Comm. Math. Phys., 316 (2012), 723-761.

[14] S. Das, P. Das, P. Das, Dynamics and control of multidrug-resistant bacterial infection in hospital with multiple delays, Comm. Nonl. Sci. Numer. Simul., 89 (2020), 105279.

[15] B. Dong, J. Song, Globally regularity and asymptotic behavior of modified Navier-Stokes equations with fractional dissipation, Discrete Cont. Dyn. Syst., 32 (2012), 57-79.

[16] C. Foias, O. Manley, R. Rosa, R. Temam, Navier-Stokes Equations and Turbulence, Cambridge University Press, Cambridge, 2001.

[17] R. Garrappa, E. Kaslik, On initial conditions for fractional delay differential equations, Comm. Nonl. Sci. Numer. Simul., 90 (2020), 105359.

[18] M. J. Garrido-Atienza, P. Marín-Rubio, Navier-Stokes equations with delays on unbounded domains, Nonlinear Anal. 64 (2006), 1100-1118. 
[19] J. García-Luengo, P. Marín-Rubio, J. Real, Pullback attractors in $V$ for non-autonomous 2D-NavierStokes equations and their tempered behavior, J. Differential Equations, 252(2012), 4333-4356.

[20] S. M. Guzzo, G. Planas, Existence of solutions for a class of Navier-Stokes equations with infinite delay, Appl. Analysis, 94 (2015), 840-855.

[21] P. E. Kloeden, J. A. Langa, J. Real, Pullback $V$-attractors of a three dimensional system of nonautonomous globally modified Navier-Stokes equations: Existence and finite fractal dimension, Comm. Pure Appl. Anal., 6 (2007), 937-955.

[22] P. E. Kloeden, P. Marín-Rubio, J. Real, Equivalence of invariant measures and stationary statistical solutions for the autonomous globally modified Navier-Stokes equations, Comm. Pure Appl. Anal., 8 (2009), 785-802.

[23] P. E. Kloeden, J. Valero, The weak connectedness of the attainability set of weak solutions of the 3D Navier-Stokes equations, Proc. Roy. Soc. London A, 463 (2007), 1491-1508.

[24] X. Li, W. Shen, C. Sun, Invariant measures for complex-valued dissipative dynamical systems and applications, Discrete Cont. Dyn. Syst.-B, 22 (2017), 2427-2446.

[25] L. Liu, T. Caraballo, P. Marín-Rubio, Stability results for 2D Navier-Stokes equations with unbounded delay, J. Differential Equations, 265 (2018), 5685-5708.

[26] G. Łukaszewicz, Pullback attractors and statistical solutions for 2-D Navier-Stokes equations, Discrete Cont. Dyn. Syst.-B, 9 (2008), 643-659.

[27] G. Łukaszewicz, J. Real, J. C. Robinson, Invariant measures for dissipative dynamical systems and generalised Banach limits, J. Dyn. Differential Equations, 23 (2011), 225-250.

[28] G. Łukaszewicz, J. C. Robinson, Invariant measures for non-autonomous dissipative dynamical systems, Discrete Cont. Dyn. Syst., 34 (2014), 4211-4222.

[29] P. Marín-Rubio, J. Real, Attractors for 2D-Navier-Stokes equations with delays on some unbounded domains, Nonlinear Anal., 67 (2007), 2784-2799.

[30] P. Marín-Rubio, A. M. Márquez-Durán, J. Real, Three dimensional system of globally modified Navier-Stokes equations with infinite delays, Discrete Cont. Dyn. Syst.-B, 14 (2010), 655-673.

[31] P. Marín-Rubio, A. M. Márquez-Durán, J. Real, Pullback attractors for globally modified NavierStokes equations with infinite delays, Discrete Cont. Dyn. Syst., 31 (2011), 779-796.

[32] P. Marín-Rubio, J. Real, J. Valero, Pullback attractors for a two-dimensional Navier-Stokes model in an infinite delay case, Nonlinear Anal., 74 (2011), 2012-2030.

[33] P. Marín-Rubio, J. Real, A. M. Márquez-Durán, On the convergence of solutions of globally modified Navier-Stokes equations with delays to solutions of Navier-Stokes equations with delays, $A d v$. Nonlinear Study, 11 (2011), 917-927.

[34] P. Marín-Rubio, A. M. Márquez-Durán, J. Real, Asymptotic behavior of solutions for a three dimensional system of globally modified Navier-Stokes equations with a locally Lipschitz delay term, Nonlinear Anal., 79 (2013), 68-79.

[35] A. M. Márquez-Durán, Existence and uniqueness of solutions, and pullback attractor for a system of globally modified 3D-Navier-Stokes equations with finite delay, SeMA Journal, 51 (2010), 117-124.

[36] C. B. G. Moussa, Invariant measures for multivalued semigroups, J. Math. Anal. Appl., 455 (2017), 1234-1248.

[37] M. Romito, The uniqueness of weak solutions of the globally modified Navier-Stokes equations, Adv. Nonlinear Study, 9 (2009), 425-427. 
[38] G. R. Sell and Y. You, Dynamics of Evolutionary Equations. Springer, 2002.

[39] R. Temam, Navier-Stokes equations, Theory and Numerical Analysis, 2nd ed., North Holland, Amsterdam, 1979.

[40] X. Wang, Upper-semicontinuity of stationary statistical properties of dissipative systems, Discrete Cont. Dyn. Syst., 23 (2009), 521-540.

[41] C. Zhao, L. Yang, Pullback attractor and invariant measures for the globally modified Navier-Stokes equations, Comm. Math. Sci., 15 (2017), 1565-1580.

[42] C. Zhao, G. Xue, G. Łukaszewicz, Pulback attractor and invariant measures for discrete KleinGordon-Schrödinger equations, Discrete Cont. Dyn. Syst.-B, 23 (2018), 4021-4044.

[43] C. Zhao, T. Caraballo, Asymptotic regularity of trajectory attractor and trajectory statistical solution for the 3D globally modified Navier-Stokes equations, J. Differential Equations, 266 (2019), 72057229.

[44] C. Zhao, Y. Li, T. Caraballo, Trajectory statistical solutions and Liouville type equations for evolution equations: Abstract results and applications, J. Differential Equations, 269(2020), 467-494.

[45] C. Zhao, Y. Li, Y. Sang, Using trajectory attractor to construct trajectory statistical solutions for 3D incompressible micropolar flows, Z. Angew. Math. Mech., 100(2020), e201800197.

[46] C. Zhao, Z. Song, T. Caraballo, Strong trajectory statistical solutions and Liouville type equations for dissipative Euler equations, Appl. Math. Lett., 99 (2020), 105981.

[47] C. Zhao, Y. Li, Z. Song, Trajectory statistical solutions for the 3D Navier-Stokes equations: The trajectory attractor approach, Nonlinear Anal.-RWA, 53(2020), 103077.

[48] Z. Zhu, C. Zhao, Pullback attractor and invariant measures for the three-dimensional regularized MHD equations, Discrete Cont. Dyn. Syst., 38 (2018), 1461-1477. 